\title{
Thrombospondin-1 and Cd47 Mediate Peripheral Microvascular Dysfunction Following Pulmonary Exposure to Multi-Walled Carbon Nanotubes
}

W. Kyle Mandler

Follow this and additional works at: https://researchrepository.wvu.edu/etd

\section{Recommended Citation}

Mandler, W. Kyle, "Thrombospondin-1 and Cd47 Mediate Peripheral Microvascular Dysfunction Following Pulmonary Exposure to Multi-Walled Carbon Nanotubes" (2017). Graduate Theses, Dissertations, and Problem Reports. 6153.

https://researchrepository.wvu.edu/etd/6153

This Dissertation is protected by copyright and/or related rights. It has been brought to you by the The Research Repository @ WVU with permission from the rights-holder(s). You are free to use this Dissertation in any way that is permitted by the copyright and related rights legislation that applies to your use. For other uses you must obtain permission from the rights-holder(s) directly, unless additional rights are indicated by a Creative Commons license in the record and/ or on the work itself. This Dissertation has been accepted for inclusion in WVU Graduate Theses, Dissertations, and Problem Reports collection by an authorized administrator of The Research Repository @ WVU.

For more information, please contact researchrepository@mail.wvu.edu. 
THROMBOSPONDIN-1 AND CD47 MEDIATE PERIPHERAL MICROVASCULAR DYSFUNCTION FOLLOWING PULMONARY EXPOSURE TO MULTI-WALLED CARBON NANOTUBES

W. Kyle Mandler

\author{
Dissertation submitted to the \\ School of Medicine at West Virginia University \\ In partial fulfillment of the requirements for the degree of \\ Doctor of Philosophy \\ In \\ Exercise Physiology \\ I. Mark Olfert, Ph.D., Chair \\ Timothy R. Nurkiewicz, Ph.D. \\ Dale W. Porter, Ph.D. \\ Paul D. Chantler, Ph.D. \\ Emidio E. Pistilli, Ph.D.
}

Department of Exercise Physiology

Morgantown, West Virginia

2017

Keywords: inhalation toxicology, arteriole, endothelial dysfunction, nanomaterials, particulate matter

Copyright 2017 Kyle Mandler 


\section{Abstract}

Thrombospondin-1 and cd47 mediate peripheral microvascular dysfunction following pulmonary exposure to multi-walled carbon nanotubes

W. Kyle Mandler

Pulmonary exposure to multi-walled carbon nanotubes (MWCNT) has been shown to disrupt endothelium-dependent arteriolar dilation in the peripheral microcirculation. The molecular mechanisms behind these arteriolar disruptions have yet to be fully elucidated. The secreted matricellular matrix protein thrombospondin-1 (TSP-1) is capable of moderating arteriolar vasodilation by inhibiting NO signaling at several points, including the inhibition soluble guanylate cyclase activity and eNOS activation. The central hypothesis was that TSP-1, following pulmonary exposure to MWCNT, mediates peripheral changes in dilatory capacity. To test this hypothesis, wildtype C57B6J (WT), TSP-1 knockout (TSP-1 KO) and CD47 knockout (CD47 KO) mice were exposed via lung aspiration to $50 \mu \mathrm{g}$ MWCNT or a sham dispersion medium control. Following exposure (24hrs), arteriolar characteristics and reactivity were measured in the gluteus maximus muscle using intravital microscopy (IVM) coupled with microiontophoretic delivery of acetylcholine (ACh) or sodium nitroprusside (SNP). In WT mice exposed to MWCNT, skeletal muscle TSP-1 protein increased $(p<0.05)$ $517.9 \pm 112.5 \%$ compared to sham exposed, and exhibited a $38.5 \pm 2.5 \%$ and 47.9 $\pm 7.3 \%$ decrease $(p<0.05)$ in endothelium-dependent and independent vasodilation, respectively. In contrast, TSP-1 protein was not increased following MWCNT exposure in TSP-1 KO mice and KO were protected from losses in dilatory capacity. Microvascular leukocyte-endothelium interactions were measured by leukocyte adhesion and rolling 
activity in third order venules. The WT+MWCNT group demonstrated $223.8 \pm 7.6 \%$ higher $(p<0.05)$ leukocyte rolling compared to WT+SHAM controls. TSP-1 KO and CD47 animals exposed to MWCNT showed no differences from WT+SHAM control. Total tissue total nitrite $\left(\mathrm{NO}_{2}^{-}\right)$and nitrate $\left(\mathrm{NO}_{3}^{-}\right)$a measure of nitric oxide status, was decreased $(p<0.05)$ in WT+MWCNT lung by $47.4 \pm 13.8 \%$ and plasma by $32.3 \pm 4.0$ $\%$, while not different from sham controls in the KO groups. Finally, several inflammatory cytokines, as quantified by Meso Scale Discovery, were upregulated in tissues of both TSP-1 and CD47 KO animals, but not in either of the WT groups. Taken together, these data provide the first evidence that TSP-1 is likely a central mediator of the systemic microvascular dysfunction that follows pulmonary MWCNT exposure. 
W. Kyle Mandler

Doctor of Exercise Physiology

West Virginia University, School of Medicine

Department of Exercise Physiology

2017

Committee Members

I. Mark Olfert, PhD (Chair)

Timothy R. Nurkiewicz, PhD

Dale W. Porter, Ph.D.

Paul D. Chantler, Ph.D.

Emidio Pistilli, PhD

Number of Pages: 165 


\section{Table of Contents}

Abstract

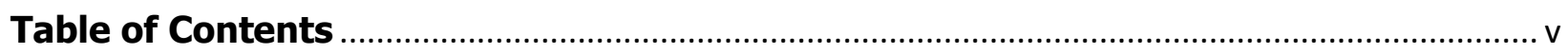

List of Abbreviations ........................................................................................................ viii

List of Tables

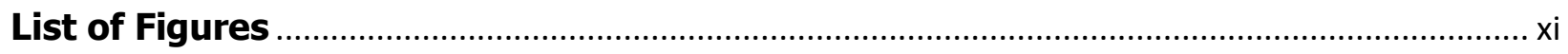

Chapter 1: Purpose, Specific Aims, Introduction, and Significance .................................

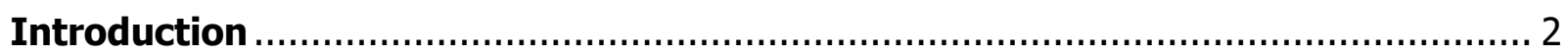

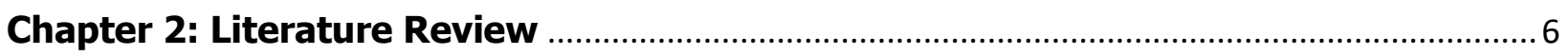

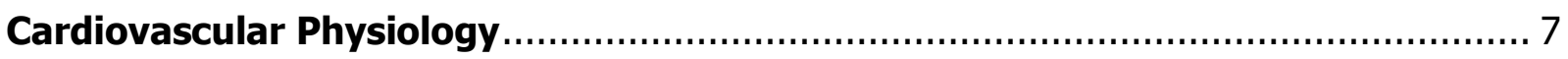

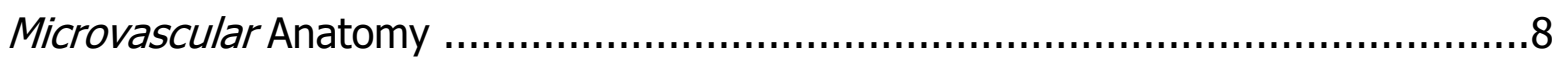

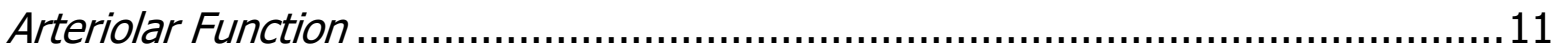

Intravital microscopy: a powerful tool for investigations of microvascular function

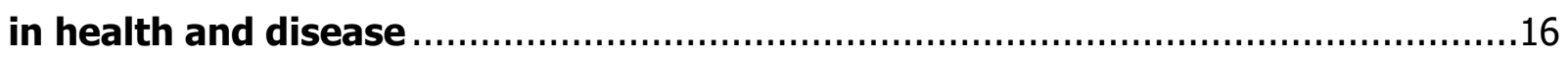

Engineered Nanomaterials represent a novel challenge for toxicologists .............18

Nanomaterial exposure elicits lung toxicity and inflammation.............................19

Pulmonary particulate matter exposure alters peripheral microvascular function. ..21

Thrombospondin-1 is a multifunctional protein with many vascular targets ........25

TSP-1 regulates smooth muscle activity through the receptor CD47 ...................26

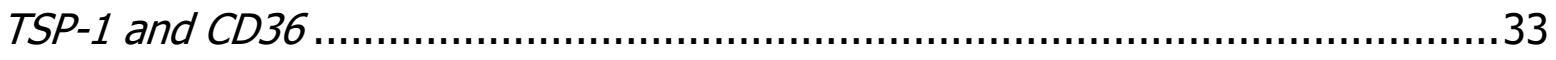

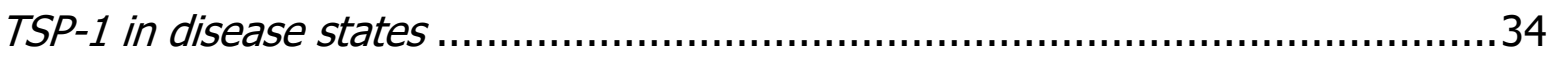

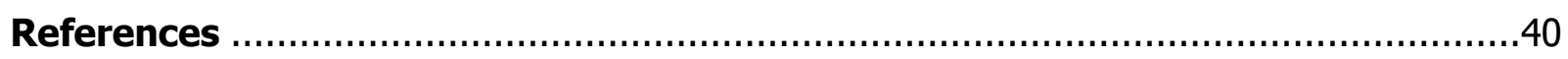

Chapter 3: Development of a mouse gluteus maximus intravital microscopy protocol

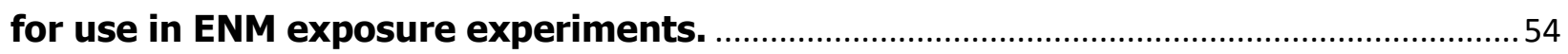

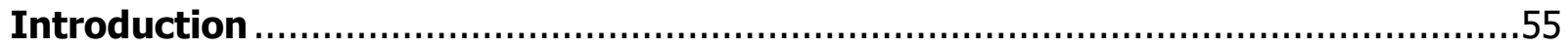




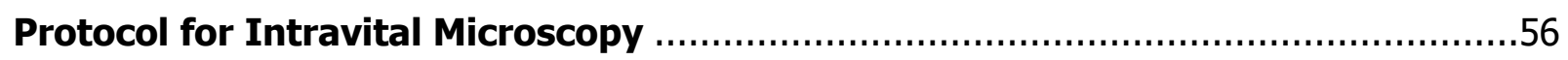

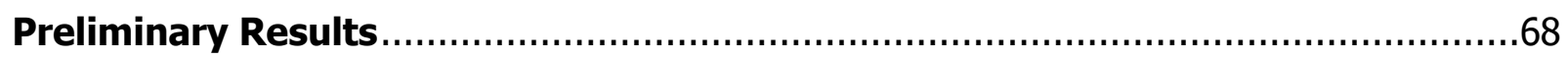

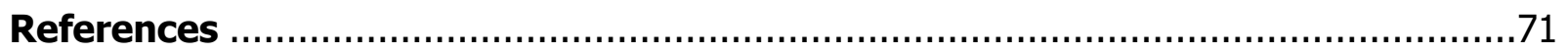

Chapter 4: Thrombospondin-1 mediates Multi-Walled Carbon Nanotube induced impairment of arteriolar dilation. .......................................................................................

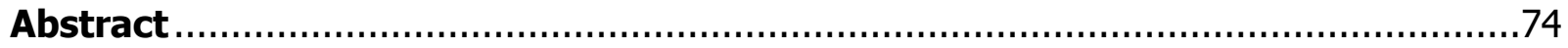

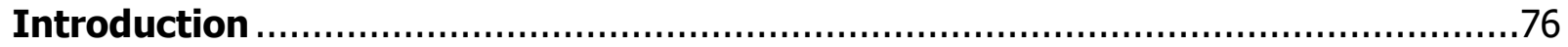

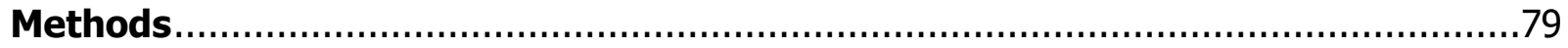

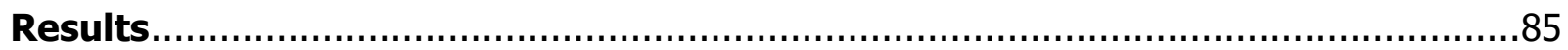

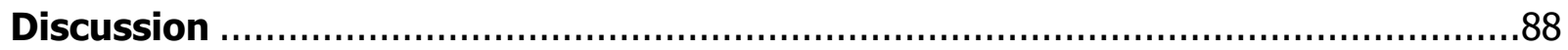

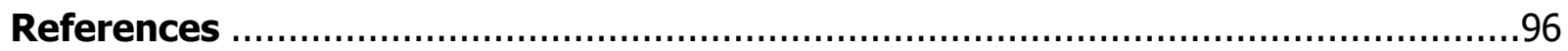

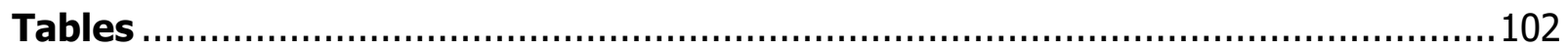

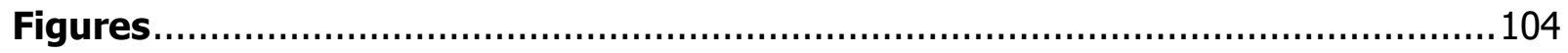

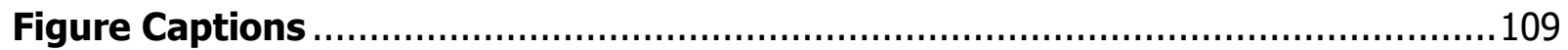

Chapter 5: Microvascular dysfunction following multi walled carbon nanotube exposure is mediated by thrombospondin-1 receptor CD47 .........................................111

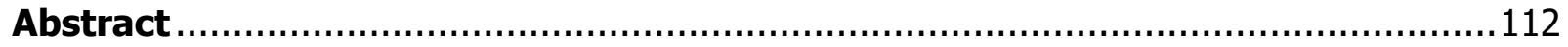

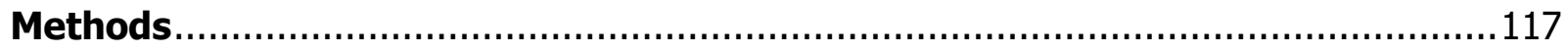

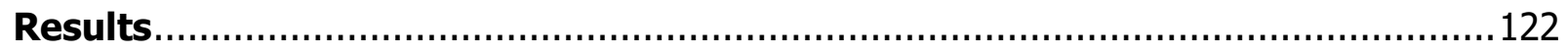

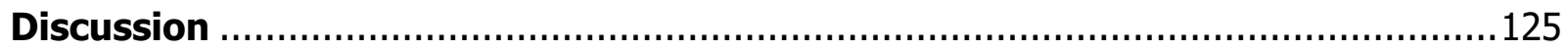

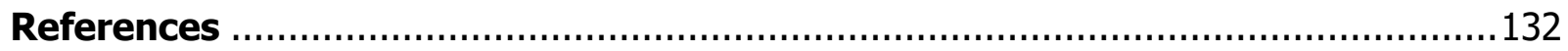

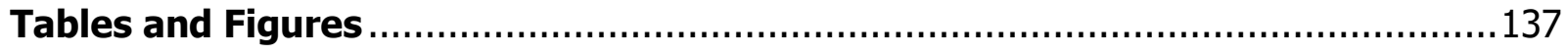

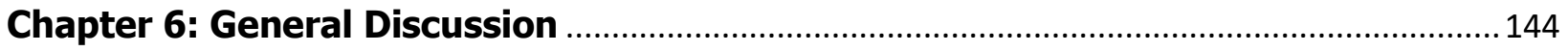

AIM 1 .

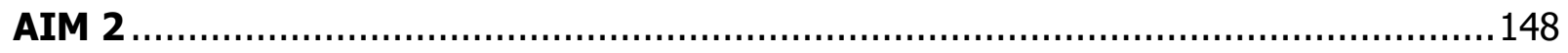

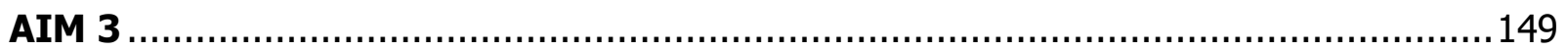




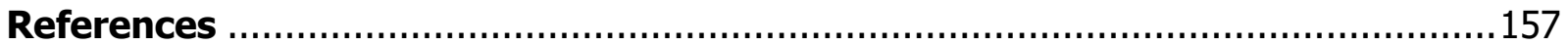




\section{List of Abbreviations}

1-Hydroxy-4-Phosphono-Oxy-2,2,6,6-Tetramethylpiperidine PPH

2,2,6,6-Tetramethylpiperidine-N-Oxyl

TEMPOL

3-(5'-Hydroxymethyl-2'-Furyl)-1-Benzylindazole

YC-1

4-Amino-Benzoic Acid Hydrazide

ABAH

Acetylcholine

ACh

Analysis Of Variance

ANOVA

Apocynin

APO

Apolipoprotein E

ApoE

Bronchoalveolar Lavage Fluid

BALF

Calcium

$\mathrm{Ca}$

Calmodulin

CaM

Cardiovascular Disease

CVD

Ca-Sensitive K Channels

BK

Caveolin

CAV

Cerium Oxide

$\mathrm{CeO}$

cGMP-Dependent Kinases

cGK

Cyclic Guanosine Monophosphate

cGMP

Cyclooxygenase

COX

Electron Paramagnetic Resonance

ESR

Endothelial Nitric Oxide Synthase

eNOS

Endothelin

ET

Engineered Nanomaterials

ENM

Guanosine-5'-Triphosphate

GTP

Heat Shock Protein 90

Hsp90

Hypoxia Inducible Factor

HIF

Inducible Nitric Oxide Synthase

iNOS

Inositol 1,4,5 Triphosphate

IP3

Interferon gamma

IFNY

Interleukin

IL-X

Intracellular Adhesion Molecule

ICAM

Intravital Microscopy

IVM

Matrix Metallopeptidase 9

MMP-9

Mean Arterial Pressure

MAP

Mouse Serum Albumin

MSA

Multi-Walled Carbon Nanotubes

MWCNT 
Myeloperoxidase

MPO

Myoendothelial Junction

MEJ

Myosin Light Chain Kinase

MLCK

NADPH Oxidase

NOX

National Institute For Occupational Safety And Health

$\mathrm{NIOSH}$

Neuronal Nitric Oxide Synthase

nNOS

$\mathrm{Ng}$-Monomethyl-L-Arginine

L-NMMA

Nicotinamide Adenine Dinucleotide Phosphate

NADPH

Nitrate

NO2-

Nitric Oxide

$\mathrm{NO}$

Nitric Oxide Synthase

NOS

Nitrite

NO3-

NO Analyzer

NOA

Particulate Matter

$P M$

Phenylephrine

PE

Phosphate-Buffered Saline

PBS

Phosphodiesterase Type V

PDE5

Physiological Salt Solution

PSS

Pulmonary Arterial Hypertension

PAH

Reactive Oxygen Species

ROS

Receptor Signal-Regulatory Protein-A

SIRP-a

Residual Oil Fly Ash

ROFA

Sodium Nitroprusside

SNP

Soluble Guanylate Cyclase

SGC

Superoxide

O2•-

Thrombospondin-1

TSP-1

Titanium Dioxide

$\mathrm{TiO} 2$

Transient Receptor Potential Cation Channel Subfamily V Member 4

TRPV4

Tumor Growth Factor B

TGF $\beta$

Tumor Necrosis Factor Alpha

TNF-a

Vanadium Chloride

$\mathrm{VCl} 3$

Vascular Adhesion Molecule

VCAM

Vascular Endothelial Growth Factor

VEGF

Vascular Endothelial Growth Factor Receptor

VEGFR2

Vascular Smooth Muscle Cell

VSMC

Wildtype

WT

Zinc Oxide

$\mathrm{ZnO}$ 


\section{List of Tables}

\section{Chapter 4}

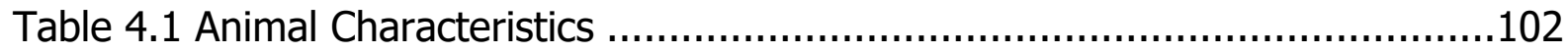

Table 4.2 Baseline Vessel Characteristics .......................................................103

\section{Chapter 5}

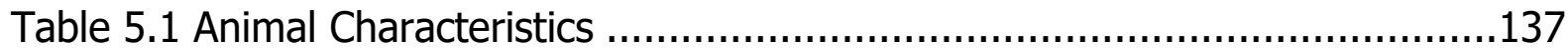

Table 5.2 Baseline Vessel Characteristics .....................................................138 


\section{List of Figures}

\section{Chapter 2}

Figure 2.1 TSP-1 inhibits vascular NO signaling pathways .29

\section{Chapter 3}

Figure 3.1 Mouse Tracheostomy 63

Figure 3.2. Skin incisions to expose the right gluteus maximus 64

Figure 3.3. Externalization and fixation of the right gluteus maximus .65

Figure 3.4. Fully externalized right gluteus maximus .66

Figure 3.5. Representative image of medial arteriolar tree. 67

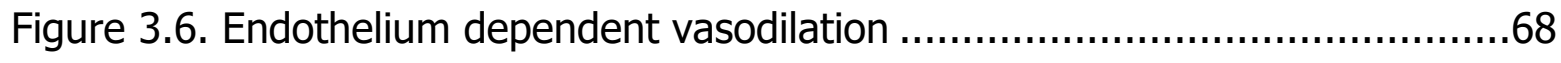

Figure 3.7. a-adrenergic sensitivity.... 69

\section{Chapter 4}

Figure 4.1 Graphical representation of intravital microscopy experiments 104

Figure 4.2 Skeletal muscle thrombospondin-1 protein level following lung multi-walled carbon nanotube exposure. 105

Figure 4.3 Arteriolar diameter following multi-walled carbon nanotube (MWCNT) aspiration ............................................................................................

Figure 4.4 Endothelium dependent and independent arteriole dilation following MWCNT aspiration 107

Figure 4.5 Skeletal muscle venule leukocyte adhesion and rolling following lung multiwalled carbon nanotube aspiration 108

\section{Chapter 5}

Figure 5.1 Endothelium dependent vasodilation in gluteus maximus arterioles ......139

Figure 5.2 Venular leukocyte adhesion and rolling activity ................................140

Figure 5.3 Blood reactive oxygen species content .........................................141

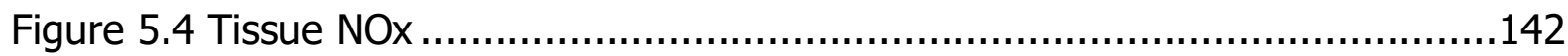

Figure 5.5 Tissue Inflammation Biomarkers …..........................................143 
Chapter 1: Purpose, Specific Aims, Introduction, and Significance 


\section{Introduction}

Purpose:

Through their modulation of blood pressure and regulation of flow to the capillaries, the arterioles play a crucial role in the maintenance of homeostasis. Arteriolar influence over these hemodynamic functions is achieved primarily through alterations in vessel diameter, which is a product of the contractile state of the smooth muscle layer. The ability of the vascular smooth muscle to react to a variety of neural, mechanical, humoral, and electrochemical stimuli is necessary to match blood flow in the capillaries to local metabolic demand. One of the most important factors influencing arteriolar reactivity is nitric oxide (NO). Produced by the vascular endothelium, NO quickly diffuses out to the smooth muscle and signals for relaxation and encouraging blood flow. An inability to produce or respond appropriately to NO can negatively impact homeostasis, and is etiologically implicated in a number of critical cardiovascular disease states, including pulmonary arterial hypertension, atherosclerosis, and peripheral and coronary vascular disease.

In addition to acute and chronic lung toxicities, pulmonary exposure to particulate matter, engineered nanomaterials, and especially multi-walled carbon nanotubes (MWCNT) have been shown to disrupt arteriolar reactivity. Evidence suggests that disruptions in NO production, signaling, and bioavailability play a role in this outcome. However, the mechanisms linking acute lung injury to peripheral changes in the vasculature have not yet been fully elucidated. The matricellular glycoprotein thrombospondin-1 (TSP-1) binding through cell surface receptors CD36 and CD47 has 
been shown to be capable of mediating NO signaling and reactivity in the vascular system through alterations in eNOS and SGC activity, and is upregulated in all of the disease states mentioned earlier. Recent evidence has put TSP-1 signaling at the forefront of possible connections between pulmonary MWCNT exposure and peripheral microvascular dysfunction.

The objective of this work was to determine the effects of TSP-1 on skeletal muscle arteriole function and its potential role in mediating microvascular dysfunction following pulmonary MWCNT exposure. The central hypothesis was that TSP-1, following pulmonary exposure to MWCNT, mediates peripheral changes in dilatory capacity.

Specific Aims:

Specific Aim 1: Develop a mouse model of skeletal muscle intravital microscopy for use in pulmonary nanomaterial exposure experiments.

Intravital microscopy is a well-established technique for examining microvascular function following nanomaterial exposure, however the bulk of this research is conducted using rats. In order to determine the involvement of TSP-1 in microvascular dysfunction, KO mice must be used. Intravital microscopy of mouse gluteus maximus muscles has been developed in other labs, but has never been used to examine the arteriolar function in the context of nanomaterial exposure or in TSP-1 knockout animals. We hypothesize that TSP-1 knockout mouse arterioles, when examined in the 
gluteus maximus using intravital microscopy, will have enhanced vasodilatory capacity, compared to WT.

\section{Specific Aim 2: Determine involvement of TSP-1 in mediating microvascular dysfunction following pulmonary MWNCT exposure}

Despite the apparent involvement of TSP-1 in influencing endothelial function and arteriolar reactivity, the effect of ENM exposure on its expression and activity and subsequent cardiovascular effects have yet to be investigated. We hypothesize that pulmonary MWCNT exposure elevates levels of TSP-1 protein in the peripheral tissues, mediating systemic arteriolar endothelial dysfunction. Mice lacking the TSP-1 gene (i.e. knockout mice) should be protected from this effect due to the lack of TSP-1 negative influence on endothelial NO production.

\section{Specific Aim 3: Determine if CD47 is responsible for mediating MWCNT/TSP- 1-induced alterations in NO signaling}

CD47 is the primary TSP-1 receptor responsible for mediating changes in vascular reactivity, with a much higher affinity and sensitivity than CD36. In some studies, CD47 appears to be necessary to mediate the anti-vasodilatory effects of TSP1. The primary objective of this aim was to determine if CD47 is involved in mediating MWCNT-induced changes in peripheral vascular function. We also sought to determine if changes in redox status in the blood and tissues were occurring that may influence 
this process, as well as identifying changes in potential inflammatory biomarkers. We hypothesize that CD47 KO animals will be protected from MWCNT-induced changes in the microvasculature, and that inflammatory markers will be elevated in WT animals, but not to the same degree as CD47 KO.

\section{Significance:}

Pulmonary exposure to particulate matter and especially nanomaterials has been shown to disrupt the critically important functions of the microvasculature. Strong evidence suggests that a disruption of vascular nitric oxide signaling is a major contributor to this dysfunction, however the mechanisms responsible for mediating this are unclear. These data will add substantially to our understanding of the role of TSP-1 in mediating peripheral microvascular dysfunction following pulmonary MWCNT exposure, and if confirmed, may offer a potential therapeutic target to prevent dysfunction in a variety of pathologies. 
Chapter 2: Literature Review 


\section{Cardiovascular Physiology}

Consisting of the heart, arteries, arterioles, capillaries, venules, veins, and the blood transported therein, proper function of the cardiovascular system contributes to the maintenance of homeostasis in health and is a source of dysfunction in disease. Through constriction, the powerful ventricles of the heart propel blood through either the pulmonary circuit where it is oxygenated in the lungs, or through the systemic circuit, where gas and nutrient exchange occurs with the tissues. Delivery of nutrients to the tissues is determined by a combination of cardiac output and peripheral control of perfusion by alterations in resistance. Cardiac output is a factor of heart rate and the volume ejected by the ventricles during each stroke (Young, 2010). Stroke volume may be influenced directly by increases in afterload caused by increased mean arterial pressure (MAP), defined below where CO = cardiac output, $\mathrm{SVR}=$ systemic vascular resistance, and CVP = central venous pressure:

$$
M A P=(C O X S V R)+C V P
$$

Changes in preload may also affect stroke volume through the Frank-Starling mechanism. Vessel diameter is a major determinant of SVR (Klabunde, 2012), this is evident in the equation below, where $R=$ resistance, $L=$ vessel length, $\eta=$ blood viscosity, and $r=$ vessel radius.

$$
R=8 L \eta /\left(\pi r^{4}\right)
$$

Due to the $4^{\text {th }}$ power relationship, vessel radius is the primary determining factor of resistance. Because their numerousness, small diameter, and relatively high muscularity, the arterioles are the greatest source of peripheral resistance in the 
circulation. Any perturbations in the ability of the arterioles to properly regulate diameter can result in improper matching of blood flow to the downstream tissues (Joussen et al., 2004), as well as alterations in systemic pressures (Landmesser and Drexler, 2007).

Of particular concern in the realm of particulate matter toxicology is the microvasculature or microcirculation, which is defined as the arterioles, capillaries, venules, and lymphatic vessels that are contained within and supply a single tissue or organ. The microvasculature is responsible for supplying nutrient and gas exchange as well as waste removal from its host tissue and must alter the rate of blood flow to match metabolic demand at any given time (Klabunde, 2012). The structure of the arterioles, capillaries, and venules help define their function.

\section{Microvascular Anatomy}

Healthy arterioles possess an intact endothelial monolayer 0.1 to $0.5 \mu \mathrm{m}$ thick, arranged longitudinally and covered on the luminal side by a thin glycocalyx (Reitsma et al., 2007). Each endothelial cell is bordered by an F-actin filament which helps transmit shear stress and mediate NO release (Fels et al., 2012). Shear stress is the force per unit area generated by the tangential force of the blood flow acting on the apical endothelial surface (Davies, 2009). Beneath the arteriolar endothelium is a basement membrane $\sim 0.1 \mu \mathrm{m}$ thick containing mostly type IV collagen, laminin, and proteoglycans (Martinez-Lemus, 2012). Surrounding the basement membrane are at least one layer of vascular smooth muscle cells, arranged perpendicular to the axis of 
blood flow. Somewhat unique to the resistance vasculature, myoendothelial junctions (MEJ) are protrusions of the endothelial cells through the basement membrane to contact the VSMCs and provide a direct mechanism of communication through gap junctions (Dora et al., 1997, Heberlein et al., 2009). The MEJ are rich in caveolae, invaginations of the lipid membrane which play an important role in mobilization and organization of signaling proteins such as $\mathrm{IP}_{3}$ receptors, ion channels, and eNOS (Straub et al., 2014). Outside of the smooth muscle is a thick layer of adventitia, composed of fibroblasts surrounded by and extracellular matrix composed primarily of collagen fibers. Nerve endings are also found embedded in the adventitia and smooth muscle. The arterioles are richly innervated by the sympathetic nervous system with many $a_{1}$ and $a_{2}$ receptors (Segal, 2005). In most vascular beds, proceeding from a main first order arteriole, the arterioles branch out into smaller successive branches into $2^{\text {nd }}, 3^{\text {rd }}$, $4^{\text {th }}$ order or until reaching the terminal arteriole immediately upstream of the capillaries. This branching scheme nomenclature is actually borrowed from the field of hydrology, where branching stream watersheds bear a striking resemblance to vascular beds (Strahler, 1957, Linderman and Boegehold, 1996). In the mouse gluteus maximus, as in most vascular beds, five branch orders can be defined by this method. In our observations, $1^{\text {st }}$ order arteriole diameter when measured under baseline conditions in the mouse gluteus maximus averaged approximately $34 \mu \mathrm{m}$, with a maximal passive diameter of about $51 \mu \mathrm{m}$, while the larger feed arterioles tended to be about $70 \mu \mathrm{m} .1^{\text {st }}$ order, also abbreviated as " $1 \mathrm{~A}$, " arterioles have higher tone, reactivity, and lumen to wall ratio compared to other vessels in the arterial tree (Pries and Secomb, 2008). 
At the distal end of the terminal capillary lies the precapillary sphincter, beyond which are many branching capillaries. In the skeletal muscle, as many as $10-15$ branching capillaries arise from a single terminal arteriole and run in parallel along skeletal muscle fibers, with occasional cross connections (Johnson, 2008). Unlike the arterioles, capillaries have no smooth muscle and consist mostly of an endothelium surrounded by basement membrane. Capillary length in skeletal muscle ranges from 100 to $400 \mu \mathrm{m}$ (Johnson, 2008). Capillary diameter tends to be smaller at the arterial side with a slight widening toward the venular side (Henquell et al., 1976). The arteriolar-side diameter tends to be just smaller than the erythrocytes, with a slight widening toward the venular side (Pries and Secomb, 2008). Capillary density can vary widely between tissues and between fiber types in skeletal muscle, with a trend toward higher density in more oxidative fibers (Qu et al., 1997). In dog gastrocnemius muscle it was observed that there were $260 \mathrm{~km}$ of capillaries, with a total area of $2 \mathrm{~m}^{2}$ per 100 g of muscle (Casley-Smith et al., 1974).

The venules mirror the arterioles in organization, although they tend to be more numerous and drain the capillary network supplied by arterioles. Venular pressures are very low, and their diameter is significantly larger than arterioles in the same order. The result of low pressure and wide vessels is generally blood velocity compared to the arterioles. The venules in skeletal muscle tend to be poorly innervated if at all, although in other tissues this is not necessarily the case. Venules represent the major site for protein exchange and leukocyte trafficking (Granger and Senchenkova, 2010a). 
Interestingly, vasodilatory and anti-vasodilatory cross-talk between venules and nearby arterioles is possible (Falcone and Bohlen, 1990).

\section{Arteriolar Function}

Owing to their distribution, anatomical size, and high smooth muscle/vessel wall ratio, the arterioles are uniquely positioned in the vascular anatomy to regulate blood flow to a greater degree than other vascular components. Flow (Q) through a single uniform vessel can be approximately described using Poiseuille's equation, where $r=$ radius, $\mathrm{I}=$ length of the vessel, $\eta=$ blood viscosity, and $\mathrm{P}=$ pressure.

$$
Q=\frac{\pi}{8} * \frac{r^{4}}{l} * \frac{1}{\eta} * \Delta P
$$

Poiseuille's equation makes several assumptions; that the vessel is uniform and cylindrical, that flow is steady and laminar, and that the fluid behaves in a Newtonian manner. Derivation of this formula reveals that flow is linearly dependent on viscosity and vessel length, but dependence on the radius is to the fourth power. Thus, changes in arterial radius can dramatically alter resistance, which is the principal method of local control of blood flow (Pries and Secomb, 2008). Under normal physiologic conditions, the arteriolar VSMCs exist in a state of constant partial contraction. The amount of VSMC contraction required to maintain this state is known as vascular tone and exists on a continuum from $0-100 \%$. Control of VSMC contraction is dependent on a complex integration of various neural, humoral, and chemical factors acting simultaneously on the endothelium and smooth muscle layers. At the most basic level, 
the contractile state of the VSMC is dependent on the concentration of intracellular free calcium ions $\left(\mathrm{Ca}^{2+}\right)$ and sensitivity of myosin light chain to those ions.

Regulation of VSMC contractile state and subsequent changes in vessel diameter is complex and dependent on a wide variety of factors and receptors. Synthesized from histidine by histidine decarboxylase, histamine stimulates hepthahelical receptors which activate PLC or adenylate cyclase (Hill et al., 1997). Prostanoids are lipid intermediates synthesized from membrane-derived arachoadonic acid by cyclooxygenases (COX) (Davidge, 2001). This family includes both prostaglandins and thromboxane.

Prostanoids interact with at least four G-protein coupled receptors, each with unique tissue distribution and biological functions including either contraction or relaxation of smooth muscle (Coleman et al., 1994). Adenosine is a nucleoside formed during ATP metabolism and exerts direct control over VSMC contraction via binding to cell membrane G-protein-coupled receptors. In skeletal muscle, vasodilation is mediated by adenosine binding to $A_{2}$ receptors, stimulating adenylate cyclase activity (Lynge and Hellsten, 2000). Adenosine may also block a-adrenoceptor mediated vasoconstriction and inhibit intrinsic tone of small arterioles (Nishigaki et al., 1991).

One of the most important local regulators of arteriolar dilation is nitric oxide (NO). NO is synthesized in the vascular endothelium from L-arginine (Palmer et al., 1988) by nitric oxide synthase (NOS). Three genes encode for distinct classes of NOS enzymes: neuronal NOS (nNOS), inducible NOS (iNOS), and endothelial NOS (eNOS). eNOS is the predominant source of NO in the vascular system and influencer of vascular function and is localized primarily in the endothelial plasma membrane when active 
(Forstermann et al., 1991, Hecker et al., 1994). Mice engineered to lack eNOS exhibit hypertension (Huang et al., 1995, Shesely et al., 1996) and impaired vasodilatory response to acetylcholine (Huang et al., 2000). eNOS is responsive to a wide range of positive and negative regulatory signals. For example, shear stress (Shen et al., 1992), acetylcholine (Stuehr et al., 1991), bradykinin (Busse et al., 1991), histamine can activate eNOS in calcium-dependent and calcium-independent pathways. Some of the principal pathways depending on calcium include the activation of transient receptor potential cation channel subfamily $V$ member 4 (TRPV4) in response to shear stress. TRPV4 activation precipitates a $\mathrm{Ca}^{2+}$ influx which can directly participate in activation of eNOS, or open calcium-activated potassium channels, generating an efflux of $\mathrm{K}^{+}$and hyperpolarization of the endothelial membrane (Mendoza et al., 2010). Hyperpolarization triggers voltage-gated calcium channels, resulting in further enhanced intracellular $\left[\mathrm{Ca}^{2+}\right]$, allowing for the formation of $\mathrm{Ca}^{2+} /$ calmodulin complex with eNOS (Busse and Mulsch, 1990). Shear stress can also activate eNOS in a calcium independent manner through the receptor tyrosine kinase vascular endothelium growth factor receptor 2 (VEGFR2) (Tzima et al., 2005), which activates the PI3K/Akt pathway, phosphorylating eNOS at serine ${ }^{1177}$ (Dimmeler et al., 1999, Fulton et al., 1999). ACh raises intracellular calcium via endothelial muscarinic receptors, whose binding activates generation of inositol 1,4,5 triphosphate (IP3) (Ishii and Kurachi, 2006), which in turn binds to receptors on the endoplasmic reticulum to release $\mathrm{Ca}^{2+}$. Acetylcholine signaling may also activate cyclooxygenase-mediated production of prostaglandins (Kellogg et al., 2005). The ultimate sensor responsible for the tight association between intracellular 
calcium and eNOS activity is the small ubiquitous protein calmodulin (CaM), which binds eNOS in a domain containing peptides 493-512 (Venema et al., 1996). Another binding protein crucial to eNOS activation is heat shock protein 90 (Hsp90). It appears that Hsp90 acts to both aid in cleavage from the inhibitory protein calveolin-1(Gratton et al., 2000), but also to prevent protease degradation during translocation to the membrane (Averna et al., 2008) Inhibition of either Hsp90 (Garcia-Cardena et al., 1998, Takahashi and Mendelsohn, 2003) or CaM (Forstermann et al., 1991) dramatically reduce eNOS activity.

Once generated, NO has an extremely short half-life in biologic systems; approximately $2 \mathrm{~ms}$. in the intravascular space, and from 0.09 to 2 seconds in the extravascular space, depending on oxygen concentration (Thomas et al., 2001). Once produced by eNOS, NO rapidly diffuses out to the surrounding smooth muscle (Malinski et al., 1993). This diffusion is aided greatly by connexin channels present in the myoendothelial junctions (Figueroa et al., 2013). Upon reaching the smooth muscle, NO activates soluble guanylate cyclase (sGC) by binding a prosthetic heme iron moiety (Ignarro et al., 1986), enhancing catalytic conversion of cyclic guanosine monophosphate (GTP) to cyclic guanosine monophosphate (cGMP) by about 400 -fold (Arnold et al., 1977, Katsuki et al., 1977). While sGC is primarily a receptor for NO, it can also be stimulated by organic nitrates and the benzimidazole derivative, YC-1 (Nossaman et al., 2012).

Raising smooth muscle cGMP concentration initiates relaxation through interaction with one of two cGMP-dependent protein groups; phosphodiesterase (PDE) 
or cGMP-dependent kinases (cGK) (Carvajal et al., 2000). cGMP also binds to some cGMP-gated ion channels which are important in other signaling processes, however their relevance in VSMC NO signaling is unclear. The principal phosphodiesterase isoform responsible for mediating VSMC relaxation is type V (PDE5). cGMP binds at the GAF A domain (Rybalkin et al., 2003) and is degraded by its constitutive action. The relationship between NO signaling, PDE5, and vasodilation is readily apparent in the mechanism of action of the erectile dysfunction drug sildenafil, which inhibits PDE5cGMP degradation, enhancing NO-mediated dilation of arterial and trabecular smooth muscle (Corbin, 2004).

Smooth muscle contractile status is modulated directly by intracellular $\left[\mathrm{Ca}^{2+}\right]$. Inositol triphosphate induces $\mathrm{Ca}^{2+}$ release from the sarcoplasmic reticulum and simultaneous influx of $\mathrm{Ca}^{2+}$ through various Ca-channels. Rising intracellular $\left[\mathrm{Ca}^{2+}\right]$ increases Ca-calmodulin interaction with myosin light chain kinase (MLCK). Now activated, MLCK is free to phosphorylate myosin light chain at serine 19 , enabling myosin-actin crossbridge formation, initiating contraction. Interestingly, Elevation of intracellular $\mathrm{Ca}^{2+}$ in the smooth muscle results in endothelial generation of $\mathrm{NO}$ in a negative feedback manner. The increasing concentration gradient drives $\mathrm{Ca}^{2+}$ through channels at the myoendothelial junction into the adjacent endothelium, stimulating NO and acting as a limit on vasoconstriction (Dora et al., 1997).

The primary pro-vasodilatory function of cGMP is mediated through binding to cGKs, which are found predominantly in lung, some brain tissues, platelets, and smooth muscle cells. cGK is activated by cGMP binding, resulting in the phosphorylation of 
several target proteins. Phosphorylation of several proteins by cGK results in an overall reduction in intracellular $\left[\mathrm{Ca}^{2+}\right]$ and a reduction in calcium sensitivity of the contractile apparatus (Carvajal et al., 2000). cGK phosphorylation drives sequestration of $\mathrm{Ca}^{2+}$ via ATP-ase calcium pumps located on the surface of the sarcoplasmic reticulum (Hurwitz et al., 1973). Additionally, cGK phosphorylation promotes opening of Ca-sensitive K channels (BK) which contributes to relaxation through hyperpolarization of the plasma membrane, reducing $\mathrm{Ca}^{2+}$ influx via voltage-gated $\mathrm{Ca}$ channels. This effect and is inhibited by tetraethylammonium and charybdotoxin, inhibitors of K-Ca channels (Archer et al., 1994). Smooth muscle sensitivity to $\mathrm{Ca}^{2+}$ can also be affected by reducing the activity of MLCK. This is achieved by dephosphorylation through myosin light chain phosphatase.

Regulation of vascular NO signaling is complex, necessary for healthy vessel function, and highly sensitive to disruption by intrinsic and extrinsic factors. Recently, growing attention has come to the effects of pulmonary exposure to particulate matter, especially that of fine $\left(\mathrm{PM}_{2.5}\right.$, diameter less than $2.5 \mu \mathrm{m}$ ) and ultrafine (one dimension smaller than $100 \mathrm{~nm}$ ) on parameters of peripheral vascular function, many of which are mediated by NO signaling.

\section{Intravital microscopy: a powerful tool for investigations of microvascular function in health and disease}

Intravital microscopy has been used extensively to study vascular responses following particulate matter exposure. Typically rats are used as the experimental 
animal of choice. Their size and tolerance of invasive surgical procedures generally is conducive to successful experiments. Vascular beds explored using rats include spinotrapezius (Linderman and Boegehold, 1996, Nurkiewicz et al., 2004, Nurkiewicz et al., 2006, Nurkiewicz et al., 2008, Nurkiewicz et al., 2009), mesentery (Minarchick et al., 2013), cremaster (Frisbee and Stepp, 2001), and uterine (Stapleton et al., 2015b). Unfortunately, genetically modified rat models are extremely limited and do not include the genes of interest for our research objectives. In order to probe molecular pathways of arteriolar function, mouse models are more readily available. Intravital microscopy of the mouse spinotrapezius has been used to study the microcirculation, but is not as well-tolerated by mice compared to rats in terms of survival rate and useful operational window (Lindner et al., 2002, Schneider et al., 2012, Guendel et al., 2013). Examination of the cremaster muscle microcirculation is much more common (Lindner et al., 2002, Bagher et al., 2011c) and has been used for transgenic studies (Bagher et al., 2011a). However, the cremaster muscle differs significantly from locomotor skeletal muscles. Electrochemically, it exhibits excessive spontaneous discharges, and the motor unit shape and firing frequency are distinct from other muscles. It also contains layers of smooth muscle interspersed between striated fibers (Kayalioglu et al., 2008). The cremaster is innervated by the following nerves; genito-femoral, ilio-inguinal, iliohypogastric, and ilio-lumbal vessels (Grant, 1966). Intravital preparation of the cremaster requires ligation of the testicular artery and vein, as well as orchiectomy, which alters pressure and flow distributions (Bagher and Segal, 2011). With these physiologic differences in mind, investigations of microvascular function in locomotor 
skeletal muscle arterioles should be considered. To date, the best preparation for this in mice is the gluteus maximus (Bearden et al., 2004, Bearden and Segal, 2004, Moore et al., 2010, Givvimani et al., 2013, Sinkler and Segal, 2014).

\section{Engineered Nanomaterials represent a novel challenge for toxicologists}

The cardiovascular implications of pulmonary exposure to particulate matter have received intense scrutiny, particularly following a position paper issued by the American Heart Association in 2004 which established particulate matter of less than $10 \mu \mathrm{m}$ $\left(\mathrm{PM}_{10}\right)$ and less than $2.5 \mu \mathrm{m}\left(\mathrm{PM}_{2.5}\right)$ in diameter as contributors to CVD (Brook et al., 2004). In particular, the paper emphasized the potency of $\mathrm{PM}_{2.5}$ in this regard owing to its ability to deposit more readily in the deep lung, compared to larger particles. The paper also noted recent research investigating ultrafine particulate matter, consisting of materials $<100 \mathrm{~nm}(0.1 \mu \mathrm{m})$ in diameter, although at the time these particles originated primarily as a byproduct of combustion processes. In the intervening years, materials science has advanced tremendously in the realm of intentionally fabricating particulate materials that fall under this ultrafine category.

Engineered nanomaterials (ENM) are a category of ultrafine particles generated industrially specifically in order to take advantage of nanoscale properties (strength/weight ratio, electrical conductivity) that are not necessarily present in their bulk counterparts. Despite not fulfilling some rather optimistic estimates of a trillion dollar market by 2015 (Roco, 2001), nanomaterials nonetheless represent an important and growing field of material and industrial science, and are increasingly being 
incorporated in a wide variety of new materials and products. The life cycle of nanomaterials includes production, use in products, and disposal, with potential exposures occurring during each (Keller et al., 2013). As such, it is vital to fully understand any toxicities that arise from ENM exposure in as many varieties and exposure routes as is feasible. As it is the most likely route of exposure to ENM in workplace settings, pulmonary exposure to inhaled, instilled, or aspirated ENM has received the most study. Other commonly investigated routes of exposure include ingestion (in part as a secondary route of exposure during clearance of inhaled ENM and dermal (commonly investigated in the context of sun screen formulations of titanium dioxide $\left(\mathrm{TiO}_{2}\right)$ and zinc oxide ( $\left.\mathrm{ZnO}\right)$ ) (Stapleton et al., 2012b).

\section{Nanomaterial exposure elicits lung toxicity and inflammation}

Because of their unique size, ENM and especially MWCNT are capable of reaching the deep lung. Pharyngeal aspiration of MWCNT results in $18 \%, 81.6 \%$, and $0.6 \%$ of MWCNT lung burden in the airway, alveolar, and sub-plural regions, respectively (Mercer et al., 2010). Once introduced to the lungs, ENM can generate toxicity through several mechanisms including reactive oxygen species (ROS) generation/oxidative stress, inflammation, perturbation of phagocytosis, and DNA damage, among others (Nel et al., 2006). Some nanoparticles are capable of rapidly ( $<1$ minutes) translocating into the circulation and depositing in organs (Nemmar et al., 2002).

Extensive work has been done to characterize the pulmonary effects of two of the most common ENM, MWCNT and nano-TiO 2 . A multi-laboratory evaluation of in 
vivo mouse and rat pulmonary responses to various forms of both MWCNT and $\mathrm{TiO}_{2}$ provides some of the best characterization of ENM lung toxicity (Bonner et al., 2013). The authors observed increased percentages of neutrophils in the bronchoalveolar lavage fluid (BALF), inflammatory lesions at alveolar duct bifurcations, and activated alveolar macrophages with numerous MWCNT inclusions in samples collected from animals exposed to both materials 1 day post-exposure. The authors noted that one of the $\mathrm{TiO}_{2}$ species in particular, the anatase nanobelt form, elicited more lung toxicity, similar to that observed in the MWCNT-exposed groups. The authors speculate that this enhanced toxicity was due in part to the higher ratio of length to width, known also as the aspect ratio, of this particular nanomaterial. It should be noted that comparisons between nanoparticles should incorporated differences in physical characteristics such as aspect ratio, surface area, or reactivity. Exposures to similar amounts of particles as measured by mass or particle number may result in different toxicities if one has a substantially different in one or more of these factors (Donaldson et al., 2011). Timecourse and exposure dose are also important factors in ENM-mediated pulmonary toxicity. Following single aspiration exposures of occupationally-relevant MWCNT aspiration doses $(10,20,40$, or $80 \mu \mathrm{g})$, BALF was collected from C57BL/6J mice at 1,7 , 28 and 56 days post-exposure. Markers of inflammation peaked at 7 days postexposure and had mostly returned to baseline by 56 days. The authors also observed fibrosis and granuloma formation as soon as 7 days post exposure and persisted through the study period. 16 weeks of repeated MWCNT exposure ( $40 \mu \mathrm{g} /$ week) in apolipoprotein $\mathrm{E}(\mathrm{ApoE})^{-/-}$mice results in BALF measurements of increased total protein, 
lactate dehydrogenase activity, surfactant protein- $D$, and mucin, as well as greatly enhanced polymorphonuclear leukocyte counts (Han, 2016). While it is apparent that exposure to ENM and especially MWCNT species generates substantial pulmonary toxicity, there is increasing evidence for secondary toxicities occurring as well, including in the cardiovascular system.

Pulmonary particulate matter exposure alters peripheral microvascular function.

While there are a limited but growing number of studies investigating the effects of pulmonary MWCNT exposure on vascular outcomes, there exists a wider body of work regarding a variety of particulate matter in this context. Exposure to mountain top mining PM (primarily $\mathrm{PM}_{10}$ ) induced a $25 \%$ and $26 \%$ inhibition of endothelium dependent vasodilation via the calcium ionophore, A23187, in mesenteric and coronary arterioles, respectively (Knuckles et al., 2013). Several studies have investigated residual oil fly ash (ROFA) and $\mathrm{TiO}_{2}$ particle exposure effects on vascular function (Nurkiewicz et al., 2004, Nurkiewicz et al., 2006, Knuckles et al., 2012). Endothelium dependent vasodilation was measured in spinotrapezius arterioles of rats exposed to ROFA, $\mathrm{TiO}_{2}$, or vehicle control. The $\mathrm{Ca}^{2+}$ ionophore elicited reduced dilation in the ROFA and $\mathrm{TiO}_{2}$ groups, while exogenous NO stimulation via sodium nitroprusside (SNP) was not inhibited (Nurkiewicz et al., 2004). Microvascular constrictive response to phenylephrine was not altered. The authors also observed enhanced myeloperoxidase (MPO) containing polymorphonuclear leukocyte adhesion and rolling activity, as well as MPO deposition in the vessel wall. These results suggest that PM exposure results in 
deficits in endothelial NO production and bioavailability while preserving smooth muscle NO sensitivity (Nurkiewicz et al., 2004, Nurkiewicz et al., 2006). In human studies, the effects of particulate matter exposure on arteriolar reactivity can be approximated by measuring blood flow during reactive hyperemia. Ultrafine particle exposure is correlated with decreased microvascular function in reactive hyperemic tests, as well as decreased lung function and increased circulating leukocytes (Olsen et al., 2014). Microvascular function is inversely related to ambient $\mathrm{PM}_{2.5}$ black carbon, NOx, and carbon monoxide and material oxidative potential (Zhang et al., 2016b).

When similar studies were performed using nanoscale versions of $\mathrm{TiO}_{2}$, inhibitions of A23187-induced arteriolar dilation were observed to be of greater severity than in previous studies with fine particles (Nurkiewicz et al., 2008). Mechanistically speaking, nanoparticle exposure reduced NO bioavailability, enhanced reactive oxygen and nitrogen species in the vessel wall, and enhanced radical generating mechanisms to a greater degree than fine particles without altering smooth muscle sensitivity to NO (Nurkiewicz et al., 2009). Similar results were observed in the sub-pericardial arterioles (LeBlanc et al., 2009, LeBlanc et al., 2010). Nano $\mathrm{TiO}_{2}$ aerosol inhalation (42.0 +/- 1.65 $\mu \mathrm{g}$ deposition), results in impaired acetylcholine responsiveness in uterine arterioles, with no change in NO sensitivity. Leukocyte adhesion and rolling activity was more than doubled (Stapleton et al., 2015b). In a follow-up study, the progeny of pregnant dams exposed to $\mathrm{TiO}_{2}$ also exhibited signs of vascular dysfunction. Coronary and uterine arteriole responsiveness to acetylcholine was significantly blunted, while the coronary arterioles also demonstrated decreased sensitivity to NO during the application of 
spermine NONOate. Mitochondrial state 3 respiration was also observed to be depressed in these vessels (Stapleton et al., 2015c).

Only a handful of studies have investigated vascular response to pulmonary MWCNT exposure. Inhalation of aerosolized MWCNT by rats resulted in changes in transmural pressure and severe impairment of acetylcholine-driven endotheliumdependent vasodilation in coronary arterioles. This impairment was maintained as long as 168 hrs. post-exposure. Responsiveness to exogenous NO and a-adrenergic activator phenylephrine remained intact (Stapleton et al., 2012a). Pulmonary exposure to MWCNT induced increases in circulating oxidative stress markers as well as procoagulant effects, with increased fibrinogen and coagulation factor VII (Luyts et al., 2014). Exposure to single-walled carbon nanotubes (SWCNT) has been shown to increase mtDNA damage and alter markers of oxidative stress (Li et al., 2007). Mouse serum obtained from animals exposed to 10 or $40 \mu \mathrm{g}$ MWCNT via oropharyngeal aspiration significantly inhibited acetylcholine responsiveness in aortic rings from naïve mice. Ablation of either CD36 in the aortas or matrix metallopeptidase 9 (MMP-9) in the exposed animals protected against this effect (Aragon et al., 2016).

Evidence for contributions of pulmonary nanotube exposure to atherosclerosis progression is mixed. A single exposure to single walled carbon nanotubes resulted in increases in markers of oxidative insults and mitochondrial DNA damage in c57BL/6 mice for as long as 60 days after exposure. Chronic biweekly exposure for 8 weeks stimulated the progression of atherosclerosis by accelerating plaque formation in $\mathrm{ApoE}^{-/-}$ mice fed an atherogenic diet 16 weeks of $40 \mu \mathrm{g} /$ week MWCNT exposure in ApoE-/- 
mice did not alter progression of atherosclerotic lesions, despite indications of lung toxicity (Li et al., 2007). Changes in diet do not seem to potentiate any apparent atherogenic effects of MWCNT exposure. 10 weeks of repeated pulmonary exposures (4 or $40 \mu \mathrm{g}$ / week) in $\mathrm{ApoE}^{-/-}$mice fed a western style resulted in increased in aortic arch thickening, suggesting artery remodeling. However, no acceleration in plaque progression in the aorta or brachiocephalic arteries were observed in histopathology sections (Christophersen et al., 2016).

As of yet it is unclear what factors relate the injury in the lung caused by exposure to nanomaterials to peripheral vascular dysfunction. Several hypotheses have been proposed to explain this phenomenon. Because of their extreme small size, ENM are capable of escaping the lung and translocating to various tissues via the circulation (Stapleton et al., 2012a). It is possible that direct interactions between ENM and the vasculature result in dysfunction. Indeed, during in vitro studies wherein vascular endothelial cells are incubated in solutions containing ENM, inflammation, reactive oxygen species generation, and NO signaling disruptions are often observed (SnyderTalkington et al., 2013, Cao et al., 2014). It should be noted, however, that only a small fraction of deposited ENM escape the lung (less than $0.001 \%$ in the case of MWCNT) (Porter et al, 2009). It is currently unclear what, if any, role this fraction of translocating ENM play in mediating microvascular dysfunction.

Another possibility is that lung exposure to ENM alters autonomic outflow to the tissues, resulting in increased sympathetic activity and a pro-vasoconstrictive environment (Stapleton et al., 2015a). Evidence for this pathway, typically in the form 
of heart rate variability alterations, exists in both experimental animals (Zheng et al., 2016) and in human cohorts (Cavallari et al., 2008a, Cavallari et al., 2008b). At this time it is unclear whether this neural activation is the result of direct interaction of inhaled particles with components of the nervous system or due to indirect circulating factors of inflammation (Stapleton et al., 2015a).

While it is almost certainly a combination of several factors, one likely pathway for microvascular dysfunction following pulmonary ENM exposure is through the spillover of inflammatory proteins into the blood, such as IL-6, IL-10, MMP-9, and others (Erdely et al., 2009). One potential mediator that is gaining attention is TSP-1.

\section{Thrombospondin-1 is a multifunctional protein with many vascular targets}

Part of the six-member thrombospondin family, TSP-1 is a large ( 450kD) glycoprotein with a variety of functions (Chen et al., 2000, Lawler, 2000). First isolated as major component of stimulated a-granules in platelets, TSP-1 was originally identified as a mediator of wound healing through its ability to enhance platelet aggregation and stabilization (Baenzige.NI et al., 1971). TSP-1 acts at the cell membrane to form complexes with cytokines and membrane proteins to regulate cell adhesion, signal transduction, and transcription (Fig. 2.1) (Lawler, 2000).

TSP-1 is a homotrimer, with each monomer joined by disulfide bonds. Each monomer is organized into discrete structural and functional segments; a terminal $\mathrm{N}$ terminal domain, a region of three types of repeated sequence motifs, and a globular terminal COOH domain (Chen et al., 2000, Lawler, 2000, Carlson et al., 2008). Thrombospondin-2 contains the same structural domains, while thrombospondins $-3,-4$, and -5 are homopentamers (Carlson et al., 2008). Each domain is associated with 
regulation of distinct functions; the $\mathrm{N}$-terminal domain are associated with heparin binding, cell attachment, spreading and migration, disruption of focal contacts, and migration (Elzie and Murphy-Ullrich, 2004). Type 1 repeats regulate protein and heparin binding, cell attachment, neurite outgrowth, tumor growth factor $\beta$ (TGF $\beta$ ) activation, as well as inhibition of proliferation and angiogenesis (Guo et al., 1997, Iruela-Arispe et al., 1999, Adams and Tucker, 2000). The region of Type 3 repeats is associated with calcium, cell binding, and apoptosis (Saumet et al., 2005), while the carboxyl terminal region is associated with cell attachment, VSMC chemotaxis and platelet aggregation, and NO signaling (Gao et al., 1996, Nesselroth et al., 2001).

\section{TSP-1 regulates smooth muscle activity through the receptor CD47}

The integrin associated protein CD47 is a receptor for the C-terminal domain of TSP-1 (Gao et al., 1996) containing an extracellular IgV domain, a transmembrane domain that crosses the membrane five times, and C-terminal tail contained in the cytoplasm. The first evidence for the regulation of NO signaling by TSP-1/CD47 was provided when it was demonstrated that muscle tissue explants from TSP-1 knockout mice exhibited enhanced angiogenesis relative to WT controls (Zhou et al., 2006), and that this response was enhanced when exogenous L-arginine or NO donors were supplied (Isenberg et al., 2005a). Further evidence for the TSP/CD47 axis was provided by studies using peptides generated from the TSP-1 peptide sequence responsible for CD47-binding, located on the TSP-1 C-terminal domain, as well as recombinant proteins 
containing the entire C-terminal domain were found to inhibit NO signaling in (Gao et al., 1996, Isenberg et al., 2006a).

Activation of the TSP/CD47 signaling pathway leads to smooth muscle cell responses. Alteration of NO signaling by TSP-1, and thus vascular tone is achieved in part via inhibition of sGC activation (Fig. 2.1). Normal circulating plasma contains 100200pM TSP-1 (Bergseth et al., 2000) and is capable of regulating NO signaling at these concentrations in VSMC, in part due to a dramatic increase in sensitivity in the presence of NO (Isenberg et al., 2006b). 2.2nM TSP-1 inhibits the ability of classic SGC activators to delay thrombin-induced platelet aggregation and abrogate VSMC contraction in vitro (Miller et al., 2010). Inhibition of SGC by TSP/CD47 appears to be dependent on an increase in cytoplasmic [ $\mathrm{Ca}^{+}{ }^{+}$(Ramanathan et al., 2011). Simultaneous stimulation of VSMCs with SGC activators and exogenous TSP-1 results in significantly reduced SGC activity and enhanced contraction compared to controls with sGC stimulators alone (Miller et al., 2010).

The anti-sGC activity of TSP-1/CD47 observed in VSMC and VEC is preserved in isolated vessels and intact tissue. TSP-1 at $2.2 \mathrm{nM}$ is unable to mediate inhibition of vasodilation in CD47 KO arterial rings, while application of exogenous TSP-1 is still capable of mediating Ach-driven vasodilation in TSP-1 KO arterial rings (Bauer et al., 2010). In isolated coronary arterioles from young (4 mo.) and old (24 mo.) rats, addition of $2.2 \mathrm{nM}$ of exogenous TSP-1 increased superoxide production in both groups, and inhibited NO mediated vasodilation by $24 \%$ in the old groups. Anti-CD47 antibody restored NO-dependent vasodilation and increased coronary flow reserve in left- 
ventricular myocardium (Nevitt et al., 2016). When treated with monoclonal TSP-1 antibody or CD47-specific morpholino oligonucleotides, ischemic myocutaneous tissue flaps demonstrated increased blood flow, reduced necrosis, and enhanced tissue survival, compared to controls (Isenberg et al., 2009c). In response to challenge with exogenous NO, both TSP-1 KO and CD47 KO mice demonstrated greater decreases in blood pressure than WT, without significant differences in heart rate (Isenberg et al., 2009b).

TSP-1/CD47 also appear to be capable of inhibiting NO signaling in cGCindependent pathways. TSP-1 has been shown to inhibit agonist-stimulated interactions between eNOS and Hsp90, thus limiting eNOS activation (Fig. 2.1). Treatment of WT vessels with physiologic concentrations $(2.2 \mathrm{nM})$ as well as the signature CD47-binding domain of TSP-1, E123CaG1, inhibits ACh-stimulated vasodilation. This inhibition persisted when the same experiments were carried out with ionomycin, a specific eNOS activating drug (Bauer et al., 2010). In arteries from CD47 KO mice, this effect was lost. In this study, CD47 KO animals also exhibited lower resting MAP, SBP, and DBP compared to WT mice (Bauer et al., 2010). Upon VEGFA protein binding, VEGFR2 increases eNOS activity via phosphorylation of serine 1177 through the PI3K/Akt and PLCY/AMPK pathways. TSP-1 is capable of inhibiting VEGF-VEGFR interaction by competitive binding, as well as directly binding to circulating VEGF and speeding its removal from the extracellular space (Anon, Gupta et al., 1999). TSP-1 may interact with VEGFR in a CD47-depdendent manner (Kaur et al., 2010) (Figure 2.1). 

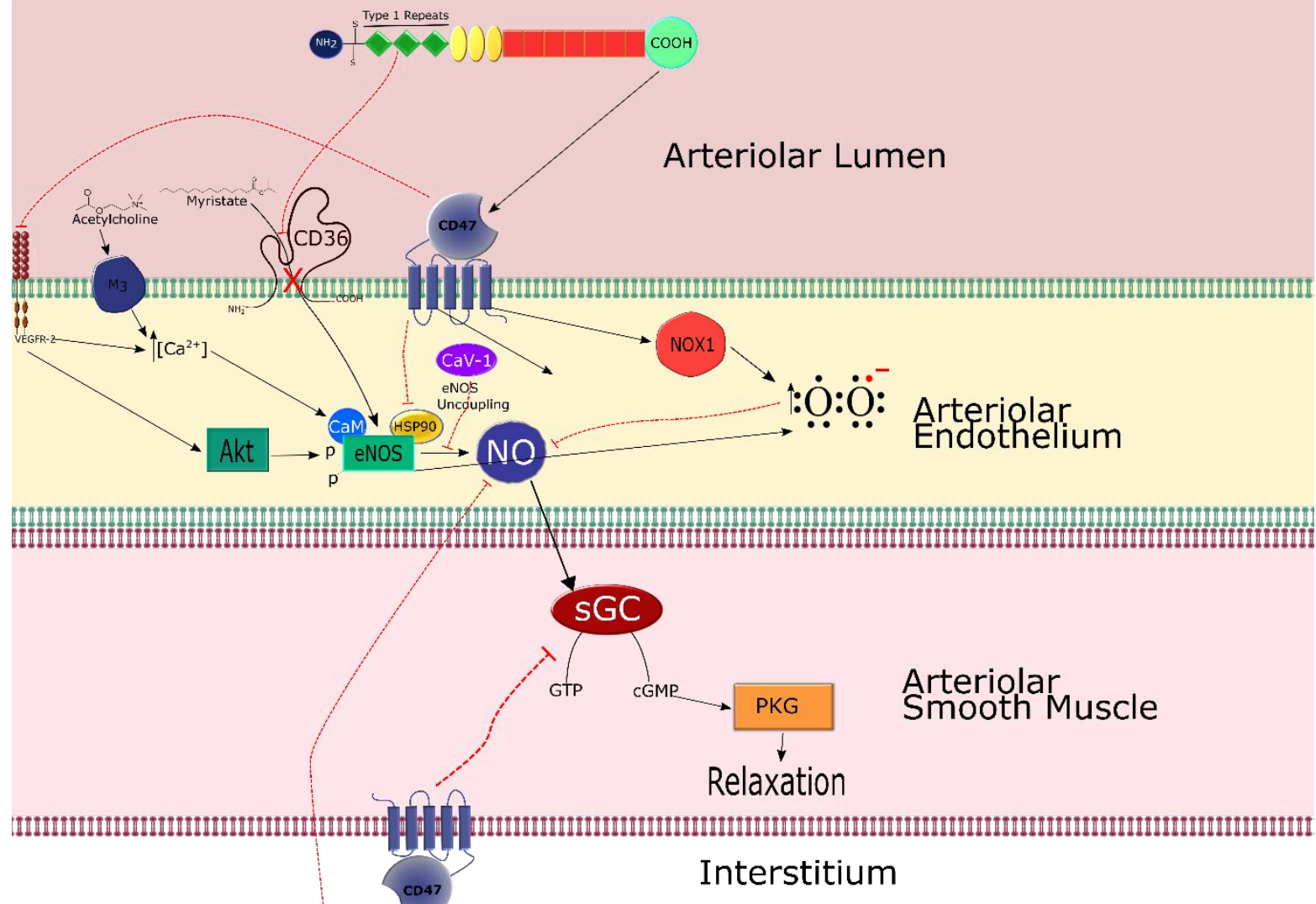

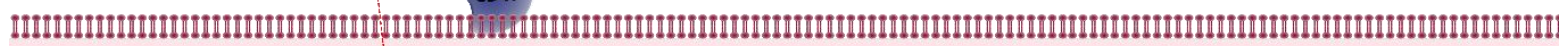
Venular Smooth Muscle

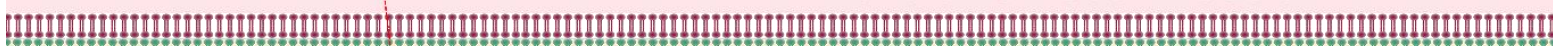

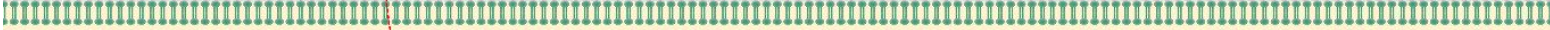

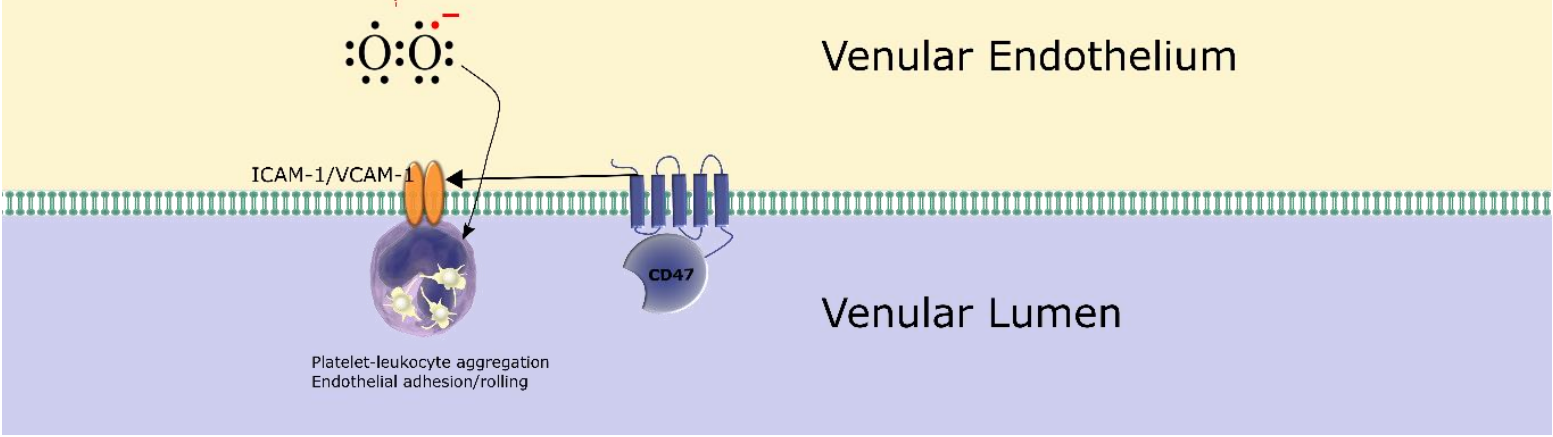


Figure 2.1. TSP-1 inhibits vascular NO signaling pathways. TSP-1 C-terminal globular domain binding to integrin associated cell surface receptor CD47 inhibits NO signaling in a variety of pathways. CD47 inhibits smooth muscle SGC activity, reducing intracellular cGMP concentrations and inhibiting relaxation. CD47 inhibits NO production by preventing HSP90 association with eNOS, causing eNOS uncoupling, as well as by inhibiting VEGFR-2 activation and inactivating circulating VEGF protein. CD47 can reduce NO bioavailability by enhancing NOX1 production of superoxide. Finally, TSP-1/CD47 can promote platelet aggregation and leukocyte adhesion to the endothelium via ICAM-1 and VCAM-1. CD36 constitutionally acts as a fatty acid translocase, allowing myristate to activate eNOS in an AMP-kinase dependent manner, TSP-1 type 1 repeat region binding inhibits this function. 
CD47 is also capable of exerting influence on vessels through non-NO pathways. Some of these potential avenues was discovered by examining the role of TSP-1 in pulmonary arterial hypertension (PAH). PAH is associated with increased expression of TSP-1 and CD47, with a more than 10x increase in circulating TSP-1 in 93 patients with PH compared to 19 control subjects (Kaiser et al., 2016). The mechanism behind the association between TSP-1/CD47 and PAH is believed to have at least two main components (Kuebler, 2017). First, it was observed that CD47 constitutively associates with caveolin-1 and that TSP-1 binding disrupts this interaction, promoting eNOS uncoupling and superoxide production (Bauer et al., 2012). Endothelial superoxide anion formation and the resultant NO scavenging contributes to increased VSMC contraction and vasoconstriction (Grunfeld et al., 1995). The second component factor tying TSP-1/CD47 to PH is through endothelin-1 (ET1) activation. ET1 and the receptor ET1 receptor A (ETA) were observed to be increased in the lungs of mice with chronic hypoxia, but not in CD47 KO mice. The authors observed that CD47 appeared to exert control of ET1 through negative regulation of the transcription factor cMyc (Bauer et al., 2010).

Finally, CD47 may also contribute to alterations in NO signaling through its capacity to act as a regulator of platelet and leukocyte recruitment. Platelet CD47 activation contributes to platelet adhesion and aggregation in times of inflammation. In VECs stimulated with TNFa, researchers were able to increase or decrease platelet adhesion by applying the CD47 agonist peptide $4 \mathrm{~N} 1 \mathrm{~K}$ or blocking monoclonal antibodies, respectively (Lagadec et al., 2003). In addition to limiting the ability of 
eNOS to generate NO, CD47 binding can cause eNOS uncoupling and production of superoxide through constitutive interaction with caveolin-1 (Bauer et al., 2012). Application of exogenous NO and/or elevation of cGMP is capable of delaying platelet aggregation during both times of high shear and static conditions. Supplying exogenous TSP-1 reverses this delay (Isenberg et al., 2008c). CD47 distributed at the apical endothelial surface is associated with upregulation of the intracellular (ICAM-1) and vascular (VCAM-1) cell adhesion molecules which promote leukocyte recruitment, adhesion, and diapedesis (Narizhneva et al., 2005). Cell adhesion molecule activation also activates NADPH oxidase-1 (NOX-1) within minutes, a necessary step of transmigration and a promoter of endothelial disorganization and permeability (DelMaschio et al., 1996, Deem and Cook-Mills, 2004).

TSP-1/CD47 signaling is capable of regulating NADPH oxidase (NOX) activity (Fig. 2.1). The NOX family of five enzyme complexes (NOX1-5) are a major generator of ROS and contribute to vascular diseases and vary in tissue distribution and activity (Cave et al., 2006, Brandes et al., 2010). In terms of vascular physiology, NOX1 and 2 are primarily sources of superoxide, while NOX4 is responsible for generating hydrogen peroxide (Dikalov et al., 2008). TSP-1 is capable of stimulating superoxide production in human VSMCs and isolated rat aortas at physiologic concentrations of TSP-1 found in humans with vascular disease $(2.2 \mathrm{nM})$ as early as 30 minutes after administration (Csanyi et al., 2012). Lower concentrations associated with healthy subjects (0.22nM) did not elicit the same response. In cells where CD47 was suppressed with antisense morpholino oligonucleotides, TSP-1-stimulated superoxide production was reduced. 
NOX1 was identified as the source of superoxide by analyzing the response of membrane fractions of VSMC with siRNA knockdown of NOX1. TSP-1 stimulated superoxide production was completely ablated in these membrane fractions. TSP-1 stimulation generated a 4-fold increase in superoxide production in endothelium-free aortic rings from WT mice compared to NOX ${ }^{-/-}$animals. Administration of NOX1 morpholino oligonucuotides in rats resulted in enhanced vasodilation and increased blood flow, even when stimulated with TSP-1, compared to vehicle control. TSP-1/CD47 activation of NOX1 appears to be dependent on surface receptor signal-regulatory protein-a (SIRP-a). TSP-1 at 2.2nM binds and activates SIRP- $a$ and its downstream signal transducer SHP1. When SIRP-a is suppressed with siRNA, TSP-1 generated superoxide was significantly reduced and vasodilation enhanced (Yao et al., 2013).

Taken together, it is apparent that TSP/CD47 signaling has wide-ranging effects on vascular NO production, signaling, bioavailability, as well as platelet aggregation and leukocyte-endothelium adhesion.

\section{TSP-1 and CD36}

While canonically recognized as the receptor associated with the antiangiogenic properties of TSP-1 (Audet et al., 2013), cell surface receptor CD36 may also play a role in the control of TSP-1 over vessel dilation. A fatty acid translocase and cholesterol uptake channel, CD36 is a ubiquitously expressed binding partner for second type I binding region of TSP-1 (Dawson et al., 1997). Like CD47, it appears that TSP-1/CD36 signaling is capable of modulating eNOS activity. Myristic acid uptake by CD36 
stimulates eNOS activity. This uptake is inhibited by $2.2 \mathrm{nM}$ TSP-1 and other CD36 binding peptides, preventing cGMP accumulation (Isenberg et al., 2007b). It is important to note, however, that CD47 sensitivity in terms of NO signaling disruption is an order of magnitude greater than that of CD36; the dissociation constant for TSP$1 / C D 47$ is $12 p M$ (Isenberg et al., 2009a) vs. $227 \pm 19 \mathrm{nM}$ for CD36.

Taken together, TSP-1 signaling through CD47 (at low concentrations), and through both CD47 and CD36 at higher concentrations appear to exert a profound antivasodilatory effect by inhibiting NO signaling, inhibiting eNOS production of NO, and by stimulating ROS generation by NOX1. It should be expected then that pathologies which generate enhanced circulating TSP-1 expression should also be associated with deficits in vascular NO function.

\section{TSP-1 in disease states}

TSP-1/CD47 signaling is enhanced in many cases etiologically linked to a variety of cardiovascular disease states. One pathology commonly associated with TSP-1 is PAH. PAH is a chronic progression of increased artery pressure, right ventricular hypertrophy, and eventually right ventricular failure, ultimately leading to death. In murine models of PAH, TSP-1 is upregulated in the lung in a hypoxia-inducible factor (HIF)-2 alpha-dependent process. When translated to clinical models, patients with endstage PAH demonstrated enhanced TSP-1 and HIF-2a protein expression in the pulmonary vasculature (Labrousse-Arias et al., 2016). In a separate study, when measured in the circulation of 93 patients with PAH, TSP-1 was observed to be more 
than 10 times higher than control $(8.64 \pm 1.05 \mathrm{nM}$ vs. $0.64 \pm 0.122 \mathrm{nM})$. Pulmonary artery pressure $(r=-0.58)$, and vascular resistance $(r=0.33)$ also correlated with TSP1 concentration. Patients who were successfully treated and survived tended to have lower levels of TSP-1, whereas TSP-1 plasma levels above $15.9 \mathrm{nM}$ was associated with all-cause mortality (Kaiser et al., 2016). TSP-1 KO mice exposed to reduced fraction of inspired oxygen (0.1) for six weeks demonstrated a resistance to development of PAH. TSP-1 KO mice had significantly lower right ventricular systolic pressures, reduced right ventricular hypertrophy, and lower right ventricular pressures in response to acute vasoconstriction compared to WT controls (Ochoa et al., 2010). It now appears that TSP-1/CD47 signaling is a major contributing factor in the development of PAH. It was observed by Rogers et al. that CD47 was upregulated in the lung and pulmonary arteries in patients with terminal PAH. CD47 KO were protected to an extent from development of right ventricular hypertension and increased right ventricular systolic pressure observed in WT animals exposed to 3 weeks of hypoxia (Rogers et al., 2017). The pathway ultimately linking upregulated TSP-1/CD47 signaling to PAH is still unclear, but evidence suggests that it is at least mediated through a constitutive interaction between CD47 and caveolin-1 (CAV1). CAV1 activation inhibits NO signaling, promotes eNOS uncoupling, and oxidative stress (Bauer et al., 2012).

TSP-1 is also considered a contributor to vascular dysfunction associated with diabetes. TSP-1 mRNA is enhanced in diabetic endothelial progenitor cells (EPCs) (Ii et al., 2006). When injected into diabetic and nondiabetic mice, EPC recruitment to the neoendothelium was reduced in both cases and associated with delayed 
reendothelialization following vascular injury. In vitro studies with diabetic EPC demonstrated decreased migration and adhesion activities, as well as reduced VEGF and eNOS expression (Ii et al., 2006). CD47 deficiency in endothelial cells improved angiogenic function and reduced endothelial cell senescence while also suppressing activation of cell cycle inhibitors and upregulating cell cycle promoters. Application of TSP-1 accelerates senescence and cell cycle arrest in a CD47-dependent manner (Gao et al., 2016).

TSP-1 is upregulated by glucose and angiotensin II and is an activator of TGF- $\beta$. In diabetic rats, development of cardiomyopathy generated by abdominal aortic coarction was abrogated by the application of a peptide antagonist of TSP-1 dependent TGF- $\beta$ activation. The progression of cardiac fibrosis was prevented and improved cardiac function was observed (Belmadani et al., 2007). In humans, higher plasma TSP1 levels are associated with diabetes and coronary artery disease. In 374 participants who had undergone coronary angiography, TSP-1 levels were higher in the diabetes and coronary artery disease group than any other group (Choi et al., 2012).

Obesity and metabolic syndrome also appear to be associated with TSP-1. In human adipose tissues and serum obtained from obese and non-obese subjects, TSP-1 expression was found to be predominantly expressed in visceral adipose, relative to the subcutaneous adipose. Visceral adiposity is a hallmark of obesity and metabolic syndrome (Wajchenberg, 2000, Varma et al., 2008). Furthermore, TSP-1 expression level was associated with abdominal obesity, hyperglycemia, and hypertension (Matsuo et al., 2015). Co-culture of adipocytes with macrophages enhances TSP-1 expression in 
each cell type, while pioglitazone treatment results in a 54\% decrease in adipose TSP-1 expression (Varma et al., 2008). The link between obesity and TSP-1 may be related to adipokines secreted by adipose tissue during obesity and inflammation. The adipokine leptin is capable of regulating TSP-1 expression in JAK2/ERK/JNK-dependent mechanism (Chavez et al., 2012). Mice fed a high fat diet (60\% fat, $20 \%$ carbohydrate) had significantly higher TSP-1 expression in perigonadal fat than in low fat diet controls. Interestingly, TSP-1 gene disruption attenuated weight gain and fat accumulation in both groups (Kong et al., 2013). This suggests that TSP-1 is not just a biomarker upregulated by obesity, but may potentiate it in a feed-forward manner.

Vessel wall injury and proliferation and migration of VSMCs are critical in the etiology of atherosclerosis. TSP-1 and CD47 expression is increased in the vessel wall in several cardiovascular diseases including obesity, diabetes, and abdominal aortic aneurysm and atherosclerosis (Stenina et al., 2003, Moura et al., 2008, Liu et al., 2015, Kojima et al., 2016). It has become clear that TSP-1 plays an active role in atheroma formation by stimulating VSMC chemotaxis and proliferation; when the carboxylterminal CD47-binding agonist peptide $4 \mathrm{n} 1 \mathrm{~K}$ is applied to both human and rat aortic smooth muscle cells, migration is stimulated. When monoclonal antibodies against CD47 were applied, chemotaxis was specifically blocked (Wang and Frazier, 1998). Concentrations of 0.1 to $30 \mu \mathrm{g} / \mathrm{mL}$ TSP-1 were sufficient to generate concomitant increases in DNA synthesis and proliferation of VSMC in a serum free environment (Patel et al., 1997). In cultured VSMCs, the presence of TSP-1 upregulated genes in 13 canonical pathways associated with atherosclerosis and an additional 4 related to 
vascular disease. When combined with hyperglycemia, 53 canonical pathways were altered (Maier et al., 2010). It appears that TSP-1-induced VSMC migration and proliferation are dependent on microRNA-21. In VSMCs incubated in miR-21 inhibitor for 20 minutes, TSP-1-induced migration was ablated and extracellular signaling kinase-1/2 phosphorylation was enhanced (Stein et al., 2014). The statin drug Pravastatin is capable of inhibiting TSP-1-induced chemotaxis, while diethylenetriamine NONOate and SNAP impeded the statin effect (Seymour et al., 2014). Dyslipidemia, often associated with atherosclerotic progression (Alberti et al., 2009), is also capable of acting synergistically with TSP-1 to promote atheroma formation. LDL at a concentration of $(175 \mu \mathrm{g} / \mathrm{ml})$ augmented chemotaxis by $30 \pm 10.4 \%$, while $\mathrm{HDL}$ at $\mathrm{HDL}(75 \mu \mathrm{g} / \mathrm{ml})$ inhibited chemotaxis by $50.9 \pm 8 \%$ in VSMCs cultured with TSP-1 (Desai et al., 2015). In diet-induced hypercholesteremic rabbits who had undergone intraluminal aortic injury, arterial wall TSP-1 was elevated 8-10 weeks later. Immunohistochemistry revealed that elevated TSP-1 was found in the extracellular matrix, and that hyperplasia was associated with levels of TSP-1 (Roth et al., 1998). TSP-1 is upregulated in human and experimental abdominal aortic aneurism, while monocytes/macrophages lacking TSP1 display reduced adhesion and migration capability. Reductions in monocyte adhesion/motility were returned to normal through the exogenous delivery of recombinant TSP-1 at a concentration of 50nM (Liu et al., 2015). When translated from in vitro work to in vivo studies, the links between TSP-1 and vascular disease persist. In plasma collected from patients with peripheral artery disease (PAD), there is a linear correlation between PAD and TSP-1 concentration (Smadja et al., 2011). TSP-1 was 
measured in 140 end stage renal disease patients and prospectively followed for 34.7 months. TSP-1 was higher in patients with preexisting clinical evidence of cardiovascular diseases. Patients in the highest tertile of circulating TSP-1 levels experienced a 6-fold higher risk of cardiovascular mortality than those in the lowest tertile (Huang et al., 2015). Administration of anti-CD47 antibodies have been shown to restore normal efferocytosis and ameliorate atherosclerosis in several mouse models. This effect appears to be driven by the pro-atherosclerotic factor TNF-a (Kojima et al., 2016).

In summary, proper arteriolar function, and especially reactivity to NO signaling is vitally important for the maintenance of homeostasis. Disruption of this signaling cascade is etiologically linked to a variety of pathologies. Pulmonary exposure to particulate matter and especially nanomaterials has been shown to disrupt arteriolar reactivity and significant evidence supports that this is mediated in a NO-dependent manner. TSP-1 is capable of mediating NO production, bioavailability, and signaling at a variety of points through its receptors CD47 and CD36. In many diseases where microvascular dysfunction, NO signaling deficits, inflammation, and reactive oxygen stress are hallmarks, TSP-1 is upregulated and anti-TSP-1 interventions have been observed to reduce symptoms. Recent investigations have put TSP-1 at the forefront of potential mediators of microvascular dysfunction following MWCNT or other particulate matter exposure. 


\section{References}

Adams, J.C. \& Tucker, R.P., 2000. The Thrombospondin Type 1 Repeat (Tsr) Superfamily: Diverse Proteins With Related Roles In Neuronal Development. Developmental Dynamics, 218, 280-299.

Alberti, K., Eckel, R.H., Grundy, S.M., Zimmet, P.Z., Cleeman, J.I., Donato, K.A., Fruchart, J.C., James, W.P.T., Loria, C.M. \& Smith, S.C., 2009. Harmonizing The Metabolic Syndrome A Joint Interim Statement Of The International Diabetes Federation Task Force On Epidemiology And Prevention; National Heart, Lung, And Blood Institute; American Heart Association; World Heart Federation; International Atherosclerosis Society; And International Association For The Study Of Obesity. Circulation, 120, 1640-1645.

Aragon, M., Erdely, A., Bishop, L., Salmen, R., Weaver, J., Liu, J., Hall, P., Eye, T., Kodali, V., Zeidler-Erdely, P., Stafflinger, J.E., Ottens, A.K. \& Campen, M.J., 2016. Mmp-9-Dependent Serum-Borne Bioactivity Caused By Multiwalled Carbon Nanotube Exposure Induces Vascular Dysfunction Via The Cd36 Scavenger Receptor. Toxicological Sciences, 150, 488-498.

Aragon, M.J., Topper, L., Tyler, C.R., Sanchez, B., Zychowski, K., Young, T., Herbert, G., Hall, P., Erdely, A., Eye, T., Bishop, L., Saunders, S.A., Muldoon, P.P., Ottens, A.K. \& Campen, M.J., 2017. Serum-Borne Bioactivity Caused By Pulmonary Multiwalled Carbon Nanotubes Induces Neuroinflammation Via Blood-Brain Barrier Impairment. Proceedings Of The National Academy Of Sciences, 114, E1968.

Archer, S.L., Huang, J.M.C., Hampl, V., Nelson, D.P., Shultz, P.J. \& Weir, E.K., 1994. Nitric-Oxide And Cgmp Cause Vasorelaxation By Activation Of A CharybdotoxinSensitive K-Channel By Cgmp-Dependent Protein-Kinase. Proceedings Of The National Academy Of Sciences Of The United States Of America, 91, 7583-7587.

Arnold, W.P., Mittal, C.K., Katsuki, S. \& Murad, F., 1977. Nitric-Oxide Activates Guanylate Cyclase And Increases Guanosine 3'-5'-Cyclic Monophosphate Levels In Various Tissue Preparations. Proceedings Of The National Academy Of Sciences Of The United States Of America, 74, 3203-3207.

Audet, G.N., Fulks, D., Stricker, J.C. \& Olfert, I.M., 2013. Chronic Delivery Of A Thrombospondin-1 Mimetic Decreases Skeletal Muscle Capillarity In Mice. Plos One, 8.

Averna, M., Stifanese, R., De Tullio, R., Passalacqua, M., Salamino, F., Pontremoli, S. \& Melloni, E., 2008. Functional Role Of Hsp90 Complexes With Endothelial NitricOxide Synthase (Enos) And Calpain On Nitric Oxide Generation In Endothelial Cells. Journal Of Biological Chemistry, 283, 29069-29076.

Baenzige.NI, Brodie, G.N. \& Majerus, P.W., 1971. Thrombin-Sensitive Protein Of Human Platelet Membranes. Proceedings Of The National Academy Of Sciences Of The United States Of America, 68, 240-\&.

Bagher, P., Davis, M.J. \& Segal, S.S., 2011a. Visualizing Calcium Responses To Acetylcholine Convection Along Endothelium Of Arteriolar Networks In 
Cx40(Bac)-Gcamp2 Transgenic Mice. American Journal Of Physiology-Heart And Circulatory Physiology, 301, H794-H802.

Bagher, P., Polo-Parada, L. \& Segal, S.S., 2011b. Microiontophoresis And Micromanipulation For Intravital Fluorescence Imaging Of The Microcirculation. Jove-Journal Of Visualized Experiments, 5.

Bagher, P. \& Segal, S.S., 2011. The Mouse Cremaster Muscle Preparation For Intravital Imaging Of The Microcirculation. Journal Of Visualized Experiments : Jove, 2874.

Bauer, E.M., Qin, Y., Miller, T.W., Bandle, R.W., Csanyi, G., Pagano, P.J., Bauer, P.M., Schnermann, J., Roberts, D.D. \& Isenberg, J.S., 2010. Thrombospondin-1 Supports Blood Pressure By Limiting Enos Activation And Endothelial-Dependent Vasorelaxation. Cardiovascular Research, 88, 471-481.

Bauer, P.M., Bauer, E.M., Rogers, N.M., Yao, M.Y., Feijoo-Cuaresma, M., Pilewski, J.M., Champion, H.C., Zuckerbraun, B.S., Calzada, M.J. \& Isenberg, J.S., 2012. Activated Cd47 Promotes Pulmonary Arterial Hypertension Through Targeting Caveolin-1. Cardiovascular Research, 93, 682-693.

Bearden, S.E., Payne, G.W., Chisty, A. \& Segal, S.S., 2004. Arteriolar Network Architecture And Vasomotor Function With Ageing In Mouse Gluteus Maximus Muscle. Journal Of Physiology-London, 561, 535-545.

Bearden, S.E. \& Segal, S.S., 2004. Motor Nerve Distribution Determines Feed Artery Control: Evidence From Mouse Gluteus Maximus Muscle. Faseb Journal, 18, Abst. 437.4-Abst. 437.4.

Belmadani, S., Bernal, J., Wei, C.C., Pallero, M.A., Dell'italia, L., Murphy-Ullrich, J.E. \& Berecek, K.H., 2007. A Thrombospondin-1 Antagonist Of Transforming Growth Factor-Beta Activation Blocks Cardiomyopathy In Rats With Diabetes And Elevated Angiotensin Ii. American Journal Of Pathology, 171, 777-789.

Bergseth, G., Lappegard, K.T., Videm, V. \& Mollnes, T.E., 2000. A Novel Enzyme Immunoassay For Plasma Thrombospondin: Comparison With BetaThromboglobulin As Platelet Activation Marker In Vitro And In Vivo. Thrombosis Research, 99, 41-50.

Bonner, J.C., Silva, R.M., Taylor, A.J., Brown, J.M., Hilderbrand, S.C., Castranova, V., Porter, D., Elder, A., Oberdorster, G., Harkema, J.R., Bramble, L.A., Kavanagh, T.J., Botta, D., Nel, A. \& Pinkerton, K.E., 2013. Interlaboratory Evaluation Of Rodent Pulmonary Responses To Engineered Nanomaterials: The Niehs Nano Go Consortium. Environmental Health Perspectives, 121, 676-682.

Brandes, R.P., Weissmann, N. \& Schroder, K., 2010. Nadph Oxidases In Cardiovascular Disease. Free Radical Biology And Medicine, 49, 687-706.

Brook, R.D., Franklin, B., Cascio, W., Hong, Y.L., Howard, G., Lipsett, M., Luepker, R., Mittleman, M., Samet, J., Smith, S.C. \& Tager, I., 2004. Air Pollution And Cardiovascular Disease - A Statement For Healthcare Professionals From The Expert Panel On Population And Prevention Science Of The American Heart Association. Circulation, 109, 2655-2671.

Busse, R., Luckhoff, A. \& Mulsch, A., 1991. Cellular Mechanisms Controlling Edrf No Formation In Endothelial-Cells. Basic Research In Cardiology, 86, 7-16. 
Busse, R. \& Mulsch, A., 1990. Calcium-Dependent Nitric-Oxide Synthesis In Endothelial Cytosol Is Mediated By Calmodulin. Febs Letters, 265, 133-136.

Cao, Y., Jacobsen, N.R., Danielsen, P.H., Lenz, A.G., Stoeger, T., Loft, S., Wallin, H., Roursgaard, M., Mikkelsen, L. \& Moller, P., 2014. Vascular Effects Of Multiwalled Carbon Nanotubes In Dyslipidemic Apoe(/) Mice And Cultured Endothelial Cells. Toxicological Sciences, 138, 104-116.

Carlson, C.B., Lawler, J. \& Mosher, D.F., 2008. Structure Of Thrombospondins. Cellular And Molecular Life Sciences, 65, 672-686.

Carvajal, J.A., Germain, A.M., Huidobro-Toro, J.P. \& Weiner, C.P., 2000. Molecular Mechanism Of Cgmp-Mediated Smooth Muscle Relaxation. Journal Of Cellular Physiology, 184, 409-420.

Casley-Smith, J.R., Green, H., Harris, J. \& P, W., 1974. The Quantitative Morphology Of Skeletal Muscle Capillaries In Relation To Permeability. Microvascular Research, 10, 43-64.

Cavallari, J.M., Eisen, E.A., Fang, S.C., Schwartz, J., Hauser, R., Herrick, R.F. \& Christiani, D.C., 2008a. Pm(2.5) Metal Exposures And Nocturnal Heart Rate Variability: A Panel Study Of Boilermaker Construction Workers. Environmental Health, 7.

Cavallari, J.M., Fang, S.C., Eisen, E.A., Schwartz, J., Hauser, R., Herrick, R.F. \& Christiani, D.C., 2008b. Time Course Of Heart Rate Variability Decline Following Particulate Matter Exposures In An Occupational Cohort. Inhalation Toxicology, 20, 415-422.

Cave, A.C., Brewer, A.C., Narayanapanicker, A., Ray, R., Grieve, D.J., Walker, S. \& Shah, A.M., 2006. Nadph Oxidases In Cardiovascular Health And Disease. Antioxidants \& Redox Signaling, 8, 691-728.

Chavez, R.J., Haney, R.M., Cuadra, R.H., Ganguly, R., Adapala, R.K., Thodeti, C.K. \& Raman, P., 2012. Upregulation Of Thrombospondin-1 Expression By Leptin In Vascular Smooth Muscle Cells Via Jak2- And Mapk-Dependent Pathways. American Journal Of Physiology-Cell Physiology, 303, C179-C191.

Chen, H., Herndon, M.E. \& Lawler, J., 2000. The Cell Biology Of Thrombospondin-1. Matrix Biology, 19, 597-614.

Choi, K.Y., Kim, D.B., Kim, M.J., Kwon, B.J., Chang, S.Y., Jang, S.W., Cho, E.J., Rho, T.H. \& Kim, J.H., 2012. Higher Plasma Thrombospondin-1 Levels In Patients With Coronary Artery Disease And Diabetes Mellitus. Korean Circulation Journal, 42, 100-106.

Christophersen, D.V., Jacobsen, N.R., Andersen, M.H.G., Connell, S.P., Barfod, K.K., Thomsen, M.B., Miller, M.R., Duffin, R., Lykkesfeldt, J., Vogel, U., Wallin, H., Loft, S., Roursgaard, M. \& Moller, P., 2016. Cardiovascular Health Effects Of Oral And Pulmonary Exposure To Multi-Walled Carbon Nanotubes In Apoe-Deficient Mice. Toxicology, 371, 29-40.

Coleman, R.A., Smith, W.L. \& Narumiya, S., 1994. International Union Of Pharmacology Classification Of Prostanoid Receptors - Properties, Distribution, And Structure Of The Receptors And Their Subtypes. Pharmacological Reviews, 46, 205-229. 
Corbin, J.D., 2004. Mechanisms Of Action Of Pde5 Inhibition In Erectile Dysfunction. International Journal Of Impotence Research, 16, S4-S7.

Csanyi, G., Yao, M., Rodriguez, A.I., Al Ghouleh, I., Sharifi-Sanjani, M., Frazziano, G., Huang, X., Kelley, E.E., Isenberg, J.S. \& Pagano, P.J., 2012. Thrombospondin-1 Regulates Blood Flow Via Cd47 Receptor Mediated Activation Of Nadph Oxidase 1. Arteriosclerosis Thrombosis And Vascular Biology, 32, 2966-+.

Davidge, S.T., 2001. Prostaglandin H Synthase And Vascular Function. Circulation Research, 89, 650-660.

Davies, P.F., 2009. Hemodynamic Shear Stress And The Endothelium In Cardiovascular Pathophysiology. Nat Clin Pract Cardiovasc Med, 6, 16-26.

Dawson, D.W., Pearce, S.F.A., Zhong, R.Q., Silverstein, R.L., Frazier, W.A. \& Bouck, N.P., 1997. Cd36 Mediates The In Vitro Inhibitory Effects Of Thrombospondin-1 On Endothelial Cells. Journal Of Cell Biology, 138, 707-717.

Deem, T.L. \& Cook-Mills, J.M., 2004. Vascular Cell Adhesion Molecule 1 (Vcam-1) Activation Of Endothelial Cell Matrix Metalloproteinases: Role Of Reactive Oxygen Species. Blood, 104, 2385-2393.

Delmaschio, A., Zanetti, A., Corada, M., Rival, Y., Ruco, L., Lampugnani, M.G. \& Dejana, E., 1996. Polymorphonuclear Leukocyte Adhesion Triggers The Disorganization Of Endothelial Cell-To-Cell Adherens Junctions. Journal Of Cell Biology, 135, 497510.

Desai, P., Stein, J.J., Siddiqui, S.A., Maier, K.G. \& Gahtan, V., 2015. Dyslipidemia Regulates Thrombospondin-1-Induced Vascular Smooth Muscle Cell Chemotaxis. Molecular And Cellular Biochemistry, 410, 85-91.

Dikalov, S.I., Dikalova, A.E., Bikineyeva, A.T., Schmidt, H., Harrison, D.G. \& Griendling, K.K., 2008. Distinct Roles Of Nox1 And Nox4 In Basal And Angiotensin IiStimulated Superoxide And Hydrogen Peroxide Production. Free Radical Biology And Medicine, 45, 1340-1351.

Dimmeler, S., Fleming, I., Fisslthaler, B., Hermann, C., Busse, R. \& Zeiher, A.M., 1999. Activation Of Nitric Oxide Synthase In Endothelial Cells By Akt-Dependent Phosphorylation. Nature, 399, 601-605.

Donaldson, K., Murphy, F., Schinwald, A., Duffin, R. \& Poland, C.A., 2011. Identifying The Pulmonary Hazard Of High Aspect Ratio Nanoparticles To Enable Their Safety-By-Design. Nanomedicine, 6, 143-156.

Dora, K.A., Doyle, M.P. \& Duling, B.R., 1997. Elevation Of Intracellular Calcium In Smooth Muscle Causes Endothelial Cell Generation Of No In Arterioles. Proceedings Of The National Academy Of Sciences Of The United States Of America, 94, 6529-6534.

Elzie, C.A. \& Murphy-Ullrich, J.E., 2004. The N-Terminus Of Thrombospondin: The Domain Stands Apart. International Journal Of Biochemistry \& Cell Biology, 36, 1090-1101.

Erdely, A., Hulderman, T., Salmen, R., Liston, A., Zeidler-Erdely, P.C., Schwegler-Berry, D., Castranova, V., Koyama, S., Kim, Y.A., Endo, M. \& Simeonova, P.P., 2009. Cross-Talk Between Lung And Systemic Circulation During Carbon Nanotube Respiratory Exposure. Potential Biomarkers. Nano Letters, 9, 36-43. 
Falcone, J.C. \& Bohlen, H.G., 1990. Edrf From Rat Intestine And Skeletal-Muscle Venules Causes Dilation Of Arterioles. American Journal Of Physiology, 258, $\mathrm{H} 1515-\mathrm{H} 1523$.

Fels, J., Jeggle, P., Kusche-Vihrog, K. \& Oberleithner, H., 2012. Cortical Actin Nanodynamics Determines Nitric Oxide Release In Vascular Endothelium. Plos One, 7, 11.

Figueroa, X.F., Lillo, M.A., Gaete, P.S., Riquelme, M.A. \& Saez, J.C., 2013. Diffusion Of Nitric Oxide Across Cell Membranes Of The Vascular Wall Requires Specific Connexin-Based Channels. Neuropharmacology, 75, 471-478.

Forstermann, U., Pollock, J.S., Schmidt, H., Heller, M. \& Murad, F., 1991. CalmodulinDependent Endothelium-Derived Relaxing Factor Nitric-Oxide Synthase Activity Is Present In The Particulate And Cytosolic Fractions Of Bovine Aortic EndothelialCells. Proceedings Of The National Academy Of Sciences Of The United States Of America, 88, 1788-1792.

Frisbee, J.C. \& Stepp, D.W., 2001. Impaired No-Dependent Dilation Of Skeletal Muscle Arterioles In Hypertensive Diabetic Obese Zucker Rats. American Journal Of Physiology-Heart And Circulatory Physiology, 281, H1304-H1311.

Fulton, D., Gratton, J.P., Mccabe, T.J., Fontana, J., Fujio, Y., Walsh, K., Franke, T.F., Papapetropoulos, A. \& Sessa, W.C., 1999. Regulation Of Endothelium-Derived Nitric Oxide Production By The Protein Kinase Akt. Nature, 399, 597-601.

Gao, A.G., Lindberg, F.P., Finn, M.B., Blystone, S.D., Brown, E.J. \& Frazier, W.A., 1996. Integrin-Associated Protein Is A Receptor For The C-Terminal Domain Of Thrombospondin. Journal Of Biological Chemistry, 271, 21-24.

Gao, Q., Chen, K.X., Gao, L., Zheng, Y. \& Yang, Y.G., 2016. Thrombospondin-1 Signaling Through Cd47 Inhibits Cell Cycle Progression And Induces Senescence In Endothelial Cells. Cell Death \& Disease, 7.

Garcia-Cardena, G., Fan, R., Shah, V., Sorrentino, R., Cirino, G., Papapetropoulos, A. \& Sessa, W.C., 1998. Dynamic Activation Of Endothelial Nitric Oxide Synthase By Hsp90. Nature, 392, 821-824.

Givvimani, S., Narayanan, N., Armaghan, F., Pushpakumar, S. \& Tyagi, S.C., 2013. Attenuation Of Conducted Vasodilation In Skeletal Muscle Arterioles During Hyperhomocysteinemia. Pharmacology, 91, 287-296.

Granger, D. \& Senchenkova, E., 2010. Anatomical Considerations. Inflammation And The Microcirculation. San Rafael, Ca: Morgan \& Claypool Life Sciences.

Grant, R.T., 1966. The Effects Of Denervation On Skeletal Muscle Blood Vessels (Rat Cremaster). Journal Of Anatomy, 100, 305-316.

Gratton, J.P., Fontana, J., O'connor, D.S., Garcia-Cardena, G., Mccabe, T.J. \& Sessa, W.C., 2000. Reconstitution Of An Endothelial Nitric-Oxide Synthase (Enos), Hsp90, And Caveolin-1 Complex In Vitro - Evidence That Hsp90 Facilitates Calmodulin Stimulated Displacement Of Enos From Caveolin-1. Journal Of Biological Chemistry, 275, 22268-22272.

Grunfeld, S., Hamilton, C.A., Mesaros, S., Mcclain, S.W., Dominiczak, A.F., Bohr, D.F. \& Malinski, T., 1995. Role Of Superoxide In The Depressed Nitric-Oxide Production 
By The Endothelium Of Genetically Hypertensive Rats. Hypertension, 26, 854857.

Guendel, A.M., Martin, K.S., Cutts, J., Foley, P.L., Bailey, A.M., Mac Gabhann, F., Cardinal, T.R. \& Peirce, S.M., 2013. Murine Spinotrapezius Model To Assess The Impact Of Arteriolar Ligation On Microvascular Function And Remodeling. JoveJournal Of Visualized Experiments.

Guo, N.H., Krutzsch, H.C., Inman, J.K. \& Roberts, D.D., 1997. Thrombospondin 1 And Type I Repeat Peptides Of Thrombospondin 1 Specifically Induce Apoptosis Of Endothelial Cells. Cancer Research, 57, 1735-1742.

Gupta, K., Gupta, P., Wild, R., Ramakrishnan, S. \& Hebbel, R.P., 1999. Binding And Displacement Of Vascular Endothelial Growth Factor (Vegf) By Thrombospondin: Effect On Human Microvascular Endothelial Cell Proliferation And Angiogenesis. Angiogenesis, 3, 147-158.

Han, S.G., 2016. Effects Of Multi-Walled Carbon Nanotubes In The Lungs And Aortas Of Apoe-Deficient Mice Fed A Normal Diet. Journal Of Nanoscience And Nanotechnology, 16, 8019-8024.

Heberlein, K., Straub, A. \& Isakson, B.E., 2009. The Myoendothelial Junction: Breaking Through The Matrix? Microcirculation (New York, N.Y. : 1994), 16, 307-322.

Hecker, M., Mulsch, A., Bassenge, E., Forstermann, U. \& Busse, R., 1994. SubcellularLocalization And Characterization Of Nitric-Oxide Synthase(S) In Endothelial-Cells - Physiological Implications. Biochemical Journal, 299, 247-252.

Henquell, L., Lacelle, P.L. \& Honig, C.R., 1976. Capillary Diameter In Rat Heart In Situ: Relation To Erythrocyte Deformability, O2 Transport, And Transmural O2 Gradients. Microvascular Research, 12, 259-274.

Hill, S.J., Ganellin, C.R., Timmerman, H., Schwartz, J.C., Shankley, N.P., Young, J.M., Schunack, W., Levi, R. \& Haas, H.L., 1997. International Union Of Pharmacology .13. Classification Of Histamine Receptors. Pharmacological Reviews, 49, 253278.

Huang, A., Sun, D., Smith, C.J., Connetta, J.A., Shesely, E.G., Koller, A. \& Kaley, G., 2000. In Enos Knockout Mice Skeletal Muscle Arteriolar Dilation To Acetylcholine Is Mediated By Edhf. American Journal Of Physiology-Heart And Circulatory Physiology, 278, H762-H768.

Huang, C.L., Jong, Y.S., Wu, Y.W., Wang, W.J., Hsieh, A.R., Chao, C.L., Chen, W.J. \& Yang, W.S., 2015. Association Of Plasma Thrombospondin-1 Level With Cardiovascular Disease And Mortality In Hemodialysis Patients. Acta Cardiologica Sinica, 31, 113-119.

Huang, P.L., Huang, Z.H., Mashimo, H., Bloch, K.D., Moskowitz, M.A., Bevan, J.A. \& Fishman, M.C., 1995. Hypertension In Mice Lacking The Gene For Endothelial Nitric-Oxide Synthase. Nature, 377, 239-242.

Hurwitz, L., Fitzpatrick, D.F., Debbas, G. \& Landon, E.J., 1973. Localization Of Calcium Pump Activity In Smooth-Muscle. Science, 179, 384-386.

Ignarro, L.J., Adams, J.B., Horwitz, P.M. \& Wood, K.S., 1986. Activation Of Soluble Guanylate-Cyclase By No-Hemoproteins Involves No-Heme Exchange - 
Comparison Of Heme-Containing And Heme-Deficient Enzyme Forms. Journal Of Biological Chemistry, 261, 4997-5002.

Ii, M., Takenaka, H., Asai, J., Ibusuki, K., Mizukami, Y., Maruyama, K., Yoon, Y.S., Wecker, A., Luedemann, C., Eaton, E., Silver, M., Thorne, T. \& Losordo, D.W., 2006. Endothelial Progenitor Thrombospondin-1 Mediates Diabetes-Induced Delay In Reendothelialization Following Arterial Injury. Circulation Research, 98, 697-704.

Iruela-Arispe, M.L., Lombardo, M., Krutzsch, H.C., Lawler, J. \& Roberts, D.D., 1999. Inhibition Of Angiogenesis By Thrombospondin-1 Is Mediated By 2 Independent Regions Within The Type 1 Repeats. Circulation, 100, 1423-1431.

Isenberg, J.S., Annis, D.S., Pendrak, M.L., Ptaszynska, M., Frazier, W.A., Mosher, D.F. \& Roberts, D.D., 2009a. Differential Interactions Of Thrombospondin-1,-2, And-4 With Cd47 And Effects On Cgmp Signaling And Ischemic Injury Responses. Journal Of Biological Chemistry, 284, 1116-1125.

Isenberg, J.S., Jia, Y.F., Fukuyama, J., Switzer, C.H., Wink, D.A. \& Roberts, D.D., 2007. Thrombospondin-1 Inhibits Nitric Oxide Signaling Via Cd36 By Inhibiting Myristic Acid Uptake. Journal Of Biological Chemistry, 282, 15404-15415.

Isenberg, J.S., Qin, Y., Maxhimer, J.B., Sipes, J.M., Despres, D., Schnermann, J., Frazier, W.A. \& Roberts, D.D., 2009b. Thrombospondin-1 And Cd47 Regulate Blood Pressure And Cardiac Responses To Vasoactive Stress. Matrix Biology, 28, 110-119.

Isenberg, J.S., Ridnour, L.A., Dimitry, J., Frazier, W.A., Wink, D.A. \& Roberts, D.D., 2006a. Cd47 Is Necessary For Inhibition Of Nitric Oxide-Stimulated Vascular Cell Responses By Thrombospondin-1. Journal Of Biological Chemistry, 281, 2606926080.

Isenberg, J.S., Ridnour, L.A., Perruccio, E.M., Espey, M.G., Wink, D.A. \& Roberts, D.D., 2005. Thrombospondin-1 Inhibits Endothelial Cell Responses To Nitric Oxide In A Cgmp-Dependent Manner. Proceedings Of The National Academy Of Sciences Of The United States Of America, 102, 13141-13146.

Isenberg, J.S., Romeo, M.J., Yu, C., Yu, C.K., Nghiem, K., Monsale, J., Rick, M.E., Wink, D.A., Frazier, W.A. \& Roberts, D.D., 2008. Thrombospondin-1 Stimulates Platelet Aggregation By Blocking The Antithrombotic Activity Of Nitric Oxide/Cgmp Signaling. Blood, 111, 613-623.

Isenberg, J.S., Shiva, S. \& Gladwin, M., 2009c. Thrombospondin-1-Cd47 Blockade And Exogenous Nitrite Enhance Ischemic Tissue Survival, Blood Flow And Angiogenesis Via Coupled No-Cgmp Pathway Activation. Nitric Oxide-Biology And Chemistry, 21, 52-62.

Isenberg, J.S., Wink, D.A. \& Roberts, D.D., 2006b. Thrombospondin-1 Antagonizes Nitric Oxide-Stimulated Vascular Smooth Muscle Cell Responses. Cardiovascular Research, 71, 785-793.

Ishii, M. \& Kurachi, Y., 2006. Muscarinic Acetylcholine Receptors. Current Pharmaceutical Design, 12, 3573-3581. 
Johnson, P.C., 2008. Overview Of The Microcirculation A2 - Tuma, Ronald F. In W.N. Durán \& K. Ley (Eds.) Microcirculation (Second Edition). San Diego: Academic Press, Xi-Xxiv.

Joussen, A.M., Poulaki, V., Le, M.L., Koizumi, K., Esser, C., Janicki, H., Schraermeyer, U., Kociok, N., Fauser, S., Kirchhof, B., Kern, T.S. \& Adamis, A.P., 2004. A Central Role For Inflammation In The Pathogenesis Of Diabetic Retinopathy. The Faseb Journal, 18, 1450-1452.

Kaiser, R., Frantz, C., Bals, R. \& Wilkens, H., 2016. The Role Of Circulating Thrombospondin-1 In Patients With Precapillary Pulmonary Hypertension. Respiratory Research, 17.

Katsuki, S., Arnold, W., Mittal, C. \& Murad, F., 1977. Stimulation Of Guanylate Cyclase By Sodium Nitroprusside, Nitroglycerin And Nitric-Oxide In Various Tissue Preparations And Comparison To Effects Of Sodium Azide And Hydroxylamine. Journal Of Cyclic Nucleotide Research, 3, 23-35.

Kaur, S., Martin-Manso, G., Pendrak, M.L., Garfield, S.H., Isenberg, J.S. \& Roberts, D.D., 2010. Thrombospondin-1 Inhibits Vegf Receptor-2 Signaling By Disrupting Its Association With Cd47. Journal Of Biological Chemistry, 285, 38923-38932.

Kayalioglu, G., Altay, B., Uyaroglu, F.G., Bademkiran, F., Uludag, B. \& Ertekin, C., 2008. Morphology And Innervation Of The Human Cremaster Muscle In Relation To Its Function. Anatomical Record-Advances In Integrative Anatomy And Evolutionary Biology, 291, 790-796.

Keller, A.A., Mcferran, S., Lazareva, A. \& Suh, S., 2013. Global Life Cycle Releases Of Engineered Nanomaterials. Journal Of Nanoparticle Research, 15, 1692.

Kellogg, D.L., Jr., Zhao, J.L., Coey, U. \& Green, J.V., 2005. Acetylcholine-Induced Vasodilation Is Mediated By Nitric Oxide And Prostaglandins In Human Skin. $J$ Appl Physiol (1985), 98, 629-32.

Klabunde, R.E., 2012. Cardiovascular Physiology Concepts, 2nd Ed. Philadelphia, Pa: Lippincott Williams \& Wilkins/Wolters Kluwer.

Knuckles, T.L., Stapleton, P.A., Minarchick, V.C., Esch, L., Mccawley, M., Hendryx, M. \& Nurkiewicz, T.R., 2013. Air Pollution Particulate Matter Collected From An Appalachian Mountaintop Mining Site Induces Microvascular Dysfunction. Microcirculation, 20, 158-169.

Knuckles, T.L., Yi, J.H., Frazer, D.G., Leonard, H.D., Chen, B.T., Castranova, V. \& Nurkiewicz, T.R., 2012. Nanoparticle Inhalation Alters Systemic Arteriolar Vasoreactivity Through Sympathetic And Cyclooxygenase-Mediated Pathways. Nanotoxicology, 6, 724-735.

Kojima, Y., Volkmer, J.P., Mckenna, K., Civelek, M., Lusis, A.J., Miller, C.L., Direnzo, D., Nanda, V., Ye, J.Q., Connolly, A.J., Schadt, E.E., Quertermous, T., Betancur, P., Maegdefessel, L., Matic, L.P., Hedin, U., Weissman, I.L. \& Leeper, N.J., 2016. Cd47-Blocking Antibodies Restore Phagocytosis And Prevent Atherosclerosis. Nature, 536, 86-+.

Kong, P., Gonzalez-Quesada, C., Li, N., Cavalera, M., Lee, D.W. \& Frangogiannis, N.G., 2013. Thrombospondin-1 Regulates Adiposity And Metabolic Dysfunction In DietInduced Obesity Enhancing Adipose Inflammation And Stimulating Adipocyte 
Proliferation. American Journal Of Physiology-Endocrinology And Metabolism, 305, E439-E450.

Kuebler, W.M., 2017. What Mediates The Effects Of Thrombospondin-1 In Pulmonary Hypertension? New Evidence For A Dual-Pronged Role Of Cd47. Cardiovascular Research, 113, 3-5.

Labrousse-Arias, D., Castillo-Gonzalez, R., Rogers, N.M., Torres-Capelli, M., Barreira, B., Aragones, J., Cogolludo, A., Isenberg, J.S. \& Calzada, M.J., 2016. Hif-2 AlphaMediated Induction Of Pulmonary Thrombospondin-1 Contributes To HypoxiaDriven Vascular Remodelling And Vasoconstriction. Cardiovascular Research, 109, 115-130.

Lagadec, P., Dejoux, O., Ticchioni, M., Cottrez, F., Johansen, M., Brown, E.J. \& Bernard, A., 2003. Involvement Of A Cd47-Dependent Pathway In Platelet Adhesion On Inflamed Vascular Endothelium Under Flow. Blood, 101, 4836-4843.

Landmesser, U. \& Drexler, H., 2007. Endothelial Function And Hypertension. Current Opinion In Cardiology, 22, 316-320.

Lawler, J., 2000. The Functions Of Thrombospondin-1 And-2. Current Opinion In Cell Biology, 12, 634-640.

Leblanc, A.J., Cumpston, J.L., Chen, B.T., Frazer, D., Castranova, V. \& Nurkiewicz, T.R., 2009. Nanoparticle Inhalation Impairs Endothelium-Dependent Vasodilation In Subepicardial Arterioles. Journal Of Toxicology And Environmental Health-Part ACurrent Issues, 72, 1576-1584.

Leblanc, A.J., Moseley, A.M., Chen, B.T., Frazer, D., Castranova, V. \& Nurkiewicz, T.R., 2010. Nanoparticle Inhalation Impairs Coronary Microvascular Reactivity Via A Local Reactive Oxygen Species-Dependent Mechanism. Cardiovascular Toxicology, 10, 27-36.

Li, Z., Hulderman, T., Salmen, R., Chapman, R., Leonard, S.S., Young, S.H., Shvedova, A., Luster, M.I. \& Simeonova, P.P., 2007. Cardiovascular Effects Of Pulmonary Exposure To Single-Wall Carbon Nanotubes. Environmental Health Perspectives, $115,377-382$.

Linderman, J.R. \& Boegehold, M.A., 1996. Arteriolar Network Growth In Rat Striated Muscle During Juvenile Maturation. International Journal Of MicrocirculationClinical And Experimental, 16, 232-239.

Lindner, J.R., Song, J., Jayaweera, A.R., Sklenar, J. \& Kaul, S., 2002. Microvascular Rheology Of Definity Microbubbles After Intra-Arterial And Intravenous Administration. Journal Of The American Society Of Echocardiography, 15, 396403.

Liu, Z., Morgan, S., Ren, J., Wang, Q., Annis, D.S., Mosher, D.F., Zhang, J., Sorenson, C.M., Sheibani, N. \& Liu, B., 2015. Thrombospondin-1 (Tsp1) Contributes To The Development Of Vascular Inflammation By Regulating Monocytic Cell Motility In Mouse Models Of Abdominal Aortic Aneurysm. Circulation Research, 117, 129141.

Luyts, K., Smulders, S., Napierska, D., Van Kerckhoven, S., Poels, K., Scheers, H., Hemmeryckx, B., Nemery, B., Hoylaerts, M.F. \& Hoet, P.H.M., 2014. Pulmonary And Hemostatic Toxicity Of Multi-Walled Carbon Nanotubes And Zinc Oxide 
Nanoparticles After Pulmonary Exposure In Bmal1 Knockout Mice. Particle And Fibre Toxicology, 11, 15.

Lynge, J. \& Hellsten, Y., 2000. Distribution Of Adenosine A(1), A(2a) And A(2b)

Receptors In Human Skeletal Muscle. Acta Physiologica Scandinavica, 169, 283290.

Maier, K.G., Han, X., Sadowitz, B., Gentile, K.L., Middleton, F.A. \& Gahtan, V., 2010. Thrombospondin-1: A Proatherosclerotic Protein Augmented By Hyperglycemia. Journal Of Vascular Surgery, 51, 1238-1247.

Malinski, T., Taha, Z., Grunfeld, S., Patton, S., Kapturczak, M. \& Tomboulian, P., 1993. Diffusion Of Nitric-Oxide In The Aorta Wall Monitored In-Situ By Porphyrinic Microsensors. Biochemical And Biophysical Research Communications, 193, 1076-1082.

Martinez-Lemus, L.A., 2012. The Dynamic Structure Of Arterioles. Basic \& Clinical Pharmacology \& Toxicology, 110, 5-11.

Matsuo, Y., Tanaka, M., Yamakage, H., Sasaki, Y., Muranaka, K., Hata, H., Ikai, I., Shimatsu, A., Inoue, M., Chun, T.H. \& Satoh-Asahara, N., 2015. Thrombospondin 1 As A Novel Biological Marker Of Obesity And Metabolic Syndrome. MetabolismClinical And Experimental, 64, 1490-1499.

Mendoza, S.A., Fang, J., Gutterman, D.D., Wilcox, D.A., Bubolz, A.H., Li, R., Suzuki, M. \& Zhang, D.X., 2010. Trpv4-Mediated Endothelial Ca2+ Influx And Vasodilation In Response To Shear Stress. American Journal Of Physiology-Heart And Circulatory Physiology, 298, H466-H476.

Mercer, R.R., Hubbs, A.F., Scabilloni, J.F., Wang, L.Y., Battelli, L.A., Schwegler-Berry, D., Castranova, V. \& Porter, D.W., 2010. Distribution And Persistence Of Pleural Penetrations By Multi-Walled Carbon Nanotubes. Particle And Fibre Toxicology, 7.

Miller, T.W., Isenberg, J.S. \& Roberts, D.D., 2010. Thrombospondin-1 Is An Inhibitor Of Pharmacological Activation Of Soluble Guanylate Cyclase. British Journal Of Pharmacology, 159, 1542-1547.

Minarchick, V.C., Stapleton, P.A., Porter, D.W., Wolfarth, M.G., Ciftyurek, E., Barger, M., Sabolsky, E.M. \& Nurkiewicz, T.R., 2013. Pulmonary Cerium Dioxide Nanoparticle Exposure Differentially Impairs Coronary And Mesenteric Arteriolar Reactivity. Cardiovascular Toxicology, 13, 323-337.

Moore, A.W., Bearden, S.E. \& Segal, S.S., 2010. Regional Activation Of Rapid Onset Vasodilatation In Mouse Skeletal Muscle: Regulation Through AlphaAdrenoreceptors. Journal Of Physiology-London, 588, 3321-3331.

Moura, R., Tjwa, M., Vandervoort, P., Van Kerckhoven, S., Holvoet, P. \& Hoylaerts, M.F., 2008. Thrombospondin-1 Deficiency Accelerates Atherosclerotic Plaque Maturation In Apoe(-/-) Mice. Circulation Research, 103, 1181-U259.

Narizhneva, N.V., Razorenova, O.V., Podrez, E.A., Chen, J.H., Chandrasekharan, U.M., Dicorleto, P.E., Plow, E.F., Topol, E.J. \& Byzova, T.V., 2005. Thrombospondin-1 Up-Regulates Expression Of Cell Adhesion Molecules And Promotes Monocyte Binding To Endothelium. Faseb Journal, 19, 1158-+.

Nel, A., Xia, T., Madler, L. \& Li, N., 2006. Toxic Potential Of Materials At The Nanolevel. Science, 311, 622-627. 
Nemmar, A., Hoet, P.H.M., Vanquickenborne, B., Dinsdale, D., Thomeer, M., Hoylaerts, M.F., Vanbilloen, H., Mortelmans, L. \& Nemery, B., 2002. Passage Of Inhaled Particles Into The Blood Circulation In Humans. Circulation, 105, 411-414. Nesselroth, S.M., Willis, A.I., Fuse, S., Olson, E.T., Lawler, J., Sumpio, B.E. \& Gahtan, V., 2001. The C-Terminal Domain Of Thrombospondin-1 Induces Vascular Smooth Muscle Cell Chemotaxis. Journal Of Vascular Surgery, 33, 595-600.

Nevitt, C., Mckenzie, G., Christian, K., Austin, J., Hencke, S., Hoying, J. \& Leblanc, A., 2016. Physiological Levels Of Thrombospondin-1 Decrease No-Dependent Vasodilation In Coronary Microvessels From Aged Rats. American Journal Of Physiology-Heart And Circulatory Physiology, 310, H1842-H1850.

Nishigaki, K., Faber, J.E. \& Ohyanagi, M., 1991. Interactions Between AlphaAdrenoceptors And Adenosine Receptors On Microvascular Smooth-Muscle. American Journal Of Physiology, 260, H1655-H1666.

Nossaman, B., Pankey, E. \& Kadowitz, P., 2012. Stimulators And Activators Of Soluble Guanylate Cyclase: Review And Potential Therapeutic Indications. Critical Care Research And Practice, 2012.

Nurkiewicz, T.R., Porter, D.W., Barger, M., Castranova, V. \& Boegehold, M.A., 2004. Particulate Matter Exposure Impairs Systemic Microvascular EndotheliumDependent Dilation. Environmental Health Perspectives, 112, 1299-1306. Nurkiewicz, T.R., Porter, D.W., Barger, M., Millecchia, L., Rao, K.M.K., Marvar, P.J., Hubbs, A.F., Castranova, V. \& Boegehold, M.A., 2006. Systemic Microvascular Dysfunction And Inflammation After Pulmonary Particulate Matter Exposure. Environmental Health Perspectives, 114, 412-419.

Nurkiewicz, T.R., Porter, D.W., Hubbs, A.F., Cumpston, J.L., Chen, B.T., Frazer, D.G. \& Castranova, V., 2008. Nanoparticle Inhalation Augments Particle-Dependent Systemic Microvascular Dysfunction. Particle And Fibre Toxicology, 5.

Nurkiewicz, T.R., Porter, D.W., Hubbs, A.F., Stone, S., Chen, B.T., Frazer, D.G., Boegehold, M.A. \& Castranova, V., 2009. Pulmonary Nanoparticle Exposure Disrupts Systemic Microvascular Nitric Oxide Signaling. Toxicological Sciences, 110, 191-203.

Ochoa, C.D., Yu, L.Y., Al-Ansari, E., Hales, C.A. \& Quinn, D.A., 2010. Thrombospondin-1 Null Mice Are Resistant To Hypoxia-Induced Pulmonary Hypertension. Journal Of Cardiothoracic Surgery, 5, 7.

Olsen, Y., Karottki, D.G., Jensen, D.M., Beko, G., Kjeldsen, B.U., Clausen, G., Hersoug, L.G., Holst, G.J., Wierzbicka, A., Sigsgaard, T., Linneberg, A., Moller, P. \& Loft, S., 2014. Vascular And Lung Function Related To Ultrafine And Fine Particles Exposure Assessed By Personal And Indoor Monitoring: A Cross-Sectional Study. Environmental Health, 13, 10.

Palmer, R.M.J., Ashton, D.S. \& Moncada, S., 1988. Vascular Endothelial-Cells Synthesize Nitric-Oxide From L-Arginine. Nature, 333, 664-666.

Patel, M.K., Lymn, J.S., Clunn, G.F. \& Hughes, A.D., 1997. Thrombospondin-1 Is A Potent Mitogen And Chemoattractant For Human Vascular Smooth Muscle Cells. Arteriosclerosis Thrombosis And Vascular Biology, 17, 2107-2114. 
Pries, A.R. \& Secomb, T.W., 2008. Chapter 1 - Blood Flow In Microvascular Networks A2 - Tuma, Ronald F. In W.N. Durán \& K. Ley (Eds.) Microcirculation (Second Edition). San Diego: Academic Press, 3-36.

Qu, Z.Q., Andersen, J.L. \& Zhou, S., 1997. Visualisation Of Capillaries In Human Skeletal Muscle. Histochemistry And Cell Biology, 107, 169-174.

Ramanathan, S., Mazzalupo, S., Boitano, S. \& Montfort, W.R., 2011. Thrombospondin-1 And Angiotensin Ii Inhibit Soluble Guanylyl Cyclase Through An Increase In Intracellular Calcium Concentration. Biochemistry, 50, 7787-7799.

Reitsma, S., Slaaf, D.W., Vink, H., Van Zandvoort, M. \& Egbrink, M., 2007. The Endothelial Glycocalyx: Composition, Functions, And Visualization. Pflugers Archiv-European Journal Of Physiology, 454, 345-359.

Roco, M.C., 2001. International Strategy For Nanotechnology Research And Development. Journal Of Nanoparticle Research, 3, 353-360.

Rogers, N.M., Sharifi-Sanjani, M., Yao, M.Y., Ghimire, K., Bienes-Martinez, R., Mutchler, S.M., Knupp, H.E., Baust, J., Novelli, E.M., Ross, M., St Croix, C., Kutten, J.C., Czajka, C.A., Sembrat, J.C., Rojas, M., Labrousse-Arias, D., Bachman, T.N., Vanderpool, R.R., Zuckerbraun, B.S., Champion, H.C., Mora, A.L., Straub, A.C., Bilonick, R.A., Calzada, M.J. \& Isenberg, J.S., 2017. TSP1-CD47 signaling is upregulated in clinical pulmonary hypertension and contributes to pulmonary arterial vasculopathy and dysfunction. Cardiovascular Research, 113, 15-29.

Roth, J.J., Gahtan, V., Brown, J.L., Gerhard, C., Swami, V.K., Rothman, V.L., Tulenko, T.N. \& Tuszynski, G.P., 1998. Thrombospondin-1 Is Elevated With Both Intimal Hyperplasia And Hypercholesterolemia. Journal Of Surgical Research, 74, 11-16.

Rybalkin, S.D., Yan, C., Bornfeldt, K.E. \& Beavo, J.A., 2003. Cyclic Gmp Phosphodiesterases And Regulation Of Smooth Muscle Function. Circulation Research, 93, 280-291.

Saumet, A., Ben Slimane, M., Lanotte, M., Lawler, J. \& Dubernard, V., 2005. Type 3 Repeat/C-Terminal Domain Of Thrombospondin-1 Triggers Caspase-Independent Cell Death Through Cd47/Alpha V Beta 3 In Promyelocytic Leukemia Nb4 Cells. Blood, 106, 658-667.

Schneider, M., Broillet, A., Tardy, I., Pochon, S., Bussat, P., Bettinger, T., Helbert, A., Costa, M. \& Tranquart, F., 2012. Use Of Intravital Microscopy To Study The Microvascular Behavior Of Microbubble-Based Ultrasound Contrast Agents. Microcirculation, 19, 245-259.

Segal, S.S., 2005. Regulation Of Blood Flow In The Microcirculation. Microcirculation, $12,33-45$.

Seymour, K., Stein, J., Han, X., Maier, K.G. \& Gahtan, V., 2014. Statins And Nitric Oxide Donors Affect Thrombospondin 1-Induced Chemotaxis. Vascular And Endovascular Surgery, 48, 470-475.

Shen, J., Luscinskas, F.W., Connolly, A., Dewey, C.F. \& Gimbrone, M.A., 1992. Fluid Shear-Stress Modulates Cytosolic Free Calcium In Vascular Endothelial-Cells. American Journal Of Physiology, 262, C384-C390. 
Shesely, E.G., Maeda, N., Kim, H.S., Desai, K.M., Krege, J.H., Laubach, V.E., Sherman, P.A., Sessa, W.C. \& Smithies, O., 1996. Elevated Blood Pressures In Mice Lacking Endothelial Nitric Oxide Synthase. Proceedings Of The National Academy Of Sciences Of The United States Of America, 93, 13176-13181.

Sinkler, S.Y. \& Segal, S.S., 2014. Aging Alters Reactivity Of Microvascular Resistance Networks In Mouse Gluteus Maximus Muscle. American Journal Of PhysiologyHeart And Circulatory Physiology, 307, H830-H839.

Smadja, D.M., D'audigier, C., Bieche, I., Evrard, S., Mauge, L., Dias, J.V., Labreuche, J., Laurendeau, I., Marsac, B., Dizier, B., Wagner-Ballon, O., Boisson-Vidal, C., Morandi, V., Duong-Van-Huyen, J.P., Bruneval, P., Dignat-George, F., Emmerich, J. \& Gaussem, P., 2011. Thrombospondin-1 Is A Plasmatic Marker Of Peripheral Arterial Disease That Modulates Endothelial Progenitor Cell Angiogenic Properties. Arteriosclerosis Thrombosis And Vascular Biology, 31, 551-559.

Snyder-Talkington, B.N., Schwegler-Berry, D., Castranova, V., Qian, Y. \& Guo, N.L., 2013. Multi-Walled Carbon Nanotubes Induce Human Microvascular Endothelial Cellular Effects In An Alveolar-Capillary Co-Culture With Small Airway Epithelial Cells. Particle And Fibre Toxicology, 10, 14.

Stapleton, P.A., Abukabda, A.B., Hardy, S.L. \& Nurkiewicz, T.R., 2015a. Xenobiotic Pulmonary Exposure And Systemic Cardiovascular Response Via Neurological Links. American Journal Of Physiology-Heart And Circulatory Physiology, 309, $\mathrm{H} 1609-\mathrm{H} 1620$.

Stapleton, P.A., Mcbride, C.R., Yi, J. \& Nurkiewicz, T.R., 2015b. Uterine Microvascular Sensitivity To Nanomaterial Inhalation: An In Vivo Assessment. Toxicology And Applied Pharmacology, 288, 420-428.

Stapleton, P.A., Minarchick, V.C., Cumpston, A.M., Mckinney, W., Chen, B.T., Sager, T.M., Frazer, D.G., Mercer, R.R., Scabilloni, J., Andrew, M.E., Castranova, V. \& Nurkiewicz, T.R., 2012a. Impairment Of Coronary Arteriolar EndotheliumDependent Dilation After Multi-Walled Carbon Nanotube Inhalation: A TimeCourse Study. International Journal Of Molecular Sciences, 13, 13781-13803.

Stapleton, P.A., Minarchick, V.C., Mccawley, M., Knuckles, T.L. \& Nurkiewicz, T.R., 2012b. Xenobiotic Particle Exposure And Microvascular Endpoints: A Call To Arms. Microcirculation, 19, 126-142.

Stapleton, P.A., Nichols, C.E., Yi, J., Mcbride, C.R., Minarchick, V.C., Shepherd, D.L., Hollander, J.M. \& Nurkiewicz, T.R., 2015c. Microvascular And Mitochondrial Dysfunction In The Female F1 Generation After Gestational Tio2 Nanoparticle Exposure. Nanotoxicology, 9, 941-951.

Stein, J.J., Iwnchukwu, C., Maier, K.G. \& Gahtan, V., 2014. Thrombospondin-1-Induced Vascular Smooth Muscle Cell Migration And Proliferation Are Functionally Dependent On Microrna-21. Surgery, 155, 228-233.

Stenina, O.I., Krukovets, I., Wang, K., Zhou, Z.M., Forudi, F., Penn, M.S., Topol, E.J. \& Plow, E.F., 2003. Increased Expression Of Thrombospondin-1 In Vessel Wall Of Diabetic Zucker Rat. Circulation, 107, 3209-3215.

Strahler, A.N., 1957. Quantitative Analysis Of Watershed Geomorphology. Eos, Transactions American Geophysical Union, 913. 
Straub, A.C., Zeigler, A.C. \& Isakson, B.E., 2014. The Myoendothelial Junction: Connections That Deliver The Message. Physiology, 29, 242-249.

Stuehr, D.J., Fasehun, O.A., Kwon, N.S., Gross, S.S., Gonzalez, J.A., Levi, R. \& Nathan, C.F., 1991. Inhibition Of Macrophage And Endothelial-Cell Nitric-Oxide Synthase By Diphenyleneiodonium And Its Analogs. Faseb Journal, 5, 98-103.

Takahashi, S. \& Mendelsohn, M.E., 2003. Calmodulin-Dependent And -Independent Activation Of Endothelial Nitric-Oxide Synthase By Heat Shock Protein 90. Journal Of Biological Chemistry, 278, 9339-9344.

Thomas, D.D., Liu, Z.P., Kantrow, S.P. \& Lancaster, J.R., 2001. The Biological Lifetime Of Nitric Oxide: Implications For The Perivascular Dynamics Of No And 0-2. Proceedings Of The National Academy Of Sciences Of The United States Of America, 98, 355-360.

Tzima, E., Irani-Tehrani, M., Kiosses, W.B., Dejana, E., Schultz, D.A., Engelhardt, B., Cao, G.Y., Delisser, H. \& Schwartz, M.A., 2005. A Mechanosensory Complex That Mediates The Endothelial Cell Response To Fluid Shear Stress. Nature, 437, 426431.

Varma, V., Yao-Borengasser, A., Bodles, A.M., Rasouli, N., Phanavanh, B., Nolen, G.T., Kern, E.M., Nagarajan, R., Spencer, H.J., Lee, M.J., Fried, S.K., Mcgehee, R.E., Peterson, C.A. \& Kern, P.A., 2008. Thrombospondin-1 Is An Adipokine Associated With Obesity, Adipose Inflammation, And Insulin Resistance. Diabetes, 57, 432439.

Venema, R.C., Sayegh, H.S., Kent, J.D. \& Harrison, D.G., 1996. Identification, Characterization, And Comparison Of The Calmodulin-Binding Domains Of The Endothelial And Inducible Nitric Oxide Synthases. Journal Of Biological Chemistry, 271, 6435-6440.

Wajchenberg, B.L., 2000. Subcutaneous And Visceral Adipose Tissue: Their Relation To The Metabolic Syndrome. Endocrine Reviews, 21, 697-738.

Wang, X.Q. \& Frazier, W.A., 1998. The Thrombospondin Receptor Cd47 (Iap) Modulates And Associates With Alpha 2 Beta 1 Integrin In Vascular Smooth Muscle Cells. Molecular Biology Of The Cell, 9, 865-874.

Yao, M., Csanyi, G., Rodriguez, A., Roberts, D., Pagano, P. \& Isenberg, J., 2013. Thrombospondin-1 (Tsp1) Impairs Vasorelaxation Via Signal Regulatory Protein (Sirp)-Alpha-Mediated Activation Of Nadph Oxidase 1 (Nox1). Faseb Journal, 27.

Young, D.B., 2010. Control Of Cardiac Output. San Rafael (Ca).

Zhang, X., Staimer, N., Tjoa, T., Gillen, D.L., Schauer, J.J., Shafer, M.M., Hasheminassab, S., Pakbin, P., Longhurst, J., Sioutas, C. \& Delfino, R.J., 2016. Associations Between Microvascular Function And Short-Term Exposure To Traffic-Related Air Pollution And Particulate Matter Oxidative Potential. Environmental Health, 15, 16.

Zheng, W., Mckinney, W., Kashon, M., Salmen, R., Castranova, V. \& Kan, H., 2016. The Influence Of Inhaled Multi-Walled Carbon Nanotubes On The Autonomic Nervous System. Particle And Fibre Toxicology, 13. 
Zhou, L., Isenberg, J.S., Cao, Z. \& Roberts, D.D., 2006. Type I Collagen Is A Molecular Target For Inhibition Of Angiogenesis By Endogenous Thrombospondin-1. Oncogene, 25, 536-545.

Chapter 3: Development of a mouse gluteus maximus intravital microscopy protocol for use in ENM exposure experiments. 


\section{Introduction}

To investigate the role TSP-1 and CD47 play in influencing microvascular reactivity following pulmonary MWCNT exposure, we decided to utilize an IVM technique to directly image intact arterioles and venules in living tissue in animals with genes for TSP-1 and CD47 knocked out. Typically, similar experiments are carried out in rats, examining the vessels of tissues including the spinotrapezius (Nurkiewicz et al., 2009, Knuckles et al., 2012), mesentery (Knuckles et al., 2013, Minarchick et al., 2015), or uterus (Stapleton et al., 2015b). However, because TSP-1 and CD47 KO animals are only available in mice, we decided to adapt a technique for IVM in the mouse gluteus maximus muscle for our purposes. IVM of the arterioles of the mouse gluteus maximus has previously been established by the Segal group (Bearden et al., 2004, Bearden and Segal, 2004, Moore et al., 2010, Sinkler and Segal, 2014). Arteriolar reactivity in TSP-1 and CD47 KO animals has been previously characterized in isolated vessels by the Isenberg group (Isenberg et al., 2006a, Isenberg et al., 2006b, Bauer et al., 2012, Csanyi et al., 2012), but reactivity using these KO strains of mice in an IVM setting has yet to be examined. In order to investigate the involvement of TSP-1 and CD47 in mediating changes in microvascular function following MWCNT exposure, it was first necessary to establish that we could satisfactorily perform IVM of the gluteus maximus microvasculature, and that we could demonstrate phenotypic differences between $\mathrm{KO}$ and WT animals. The following protocol and preliminary data represent our development of this technique at WVU. 


\section{Protocol for Intravital Microscopy}

\section{1) Anesthesia}

Anesthetization should be performed carefully, as mice are very sensitive to this barbiturate drug and it is extremely easy to overdose either to death or, much more easily, to depress the CV system enough that IVM measurements are impossible. With this in mind, going slowly with several small doses spread out over as much as 30 minutes yields good results.

a) Thiobutabarbital (Inactin, $100 \mathrm{mg} / \mathrm{kg}$, i.p.)

i) $10 \mathrm{mg}$ per $\mathrm{ml}$ of saline $=.3 \mathrm{ml}$ of solution

b) Likely interval dosing over a 30 minute period

c) $1 \mathrm{ml}$ syringe and 27 gauge for introduction of anesthesia

d) $0.05 \mathrm{ml}$ increments, wait 10 minutes between doses

\section{2) 2: Tools/Equipment}
a) \#7 forceps (extremely important for tracheostomy)
b) Vannas scissors for cutting thin muscle layers.
c) Polyethylene tubing (PE90) inch length, bevel edges
d) Silk 4-0 or 5-0 suture wire 


\section{3) Tracheostomy}

a) Use heated stage to 37 degrees Celsius

b) Shave anterior chest and neck

c) Incision with skin scissors from below chin midline to upper extremities

i) Deep to the skin there will be a thin transparent fascial layer that needs to be cut through

ii) Blunt dissect down to trachea (should be able to see cartilaginous rings in microscope)

(1) Abduct lobes of fat to expose muscle sheath around trachea

(2) Blunt dissect muscle to expose trachea

d) Suture wire 4-0 make a small loop and hold between needle driver/hemostat

e) Use \#7 forceps and go posterior to the trachea (between muscle layer and trachea) and grab the loop from the needle drivers

i) Suture wire posterior to trachea (fig. 3.1)

f) Make small incision between cartilage rings

g) Use PE90 tube, insert into trachea (about $1 \mathrm{~cm}$ )

i) Make sure both lungs are inflating by observing equal chest rise

h) Tie PE 90 tube with posterior placed suture wire

i) Cut PE90 tube to chin length

j) Suture superficial fat/skin to avoid insensible loss of fluids 


\section{4) Gluteal Maximus Externalization}

a) Position the animal as indicated in figure 3.2

b) Make an Incision slightly lateral to midline, working anteriorly first

i) Running from hind limb, up towards midline $\sim 2 \mathrm{~cm}$, and then back lateral (see fig 3.3)

c) Dissect small facial plane, avoid dissection into muscle (small amount of sub-Q tissue)

d) Can also keep things moist with saline

e) Make incision $1 \mathrm{~mm}$ medial to spine into tendon (white structure in picture)

i) Vertical incision following medial incision plane

f) Gluteus maximus striations runs laterally, with layering 1-2 mm in thickness (striations can be observed under microscope)

g) Anchoring the gluteal muscles:

i) Landmark (central vessel with branches off

ii) 1-2 $\mathrm{mm}$ from edge of incision place suture

(1) Centrally located (fig. 3.3)

(2) Tie suture with small loop so it ties onto itself rather than the muscle

(3) Secure muscle into stage and apply some traction in order to dissect farther down

(a) Avoid large blood vessels while dissecting

iii) Place 1 sutures superior to previous suture, again make a small loop during the tying process 
(1) Place the superior aspect of the muscle into traction on the stand iv) Dissect any fat away from the superior surface of the gluteal muscle in order to create as much translucency as possible

h) While dissecting the inferior medial aspect of the gluteal muscle avoid the neurovascular bundle

i) Place suture \#3 in the inferior medial aspect of the gluteal muscle (fig. 3.4)

i) While the muscle is in traction, blunt dissect the fascia away (anatomically inferior to the gluteal muscle)

i) Intermuscular fat between layers is often most highly vascular and best region to do IV microscopy

(1) While dissecting will likely rupture some small vessels

(2) 3 discrete vessel bundles to work with

(a) Superior, medial (fig. 3.5), and inferior

(b) Superior and medial are best to get images from, inferior tends to be too deep in the muscle tissue to adequately image

j) Anatomically deep (now on the exposed surface) to the muscle is a fascial layer that need to be teased away (appears like cob webs under microscope)

i) Will interfere with micro-pipet tip placement

ii) Extremely gently scrape away layer, avoid tearing muscle and vessels

k) Place stopcock grease along the edges to seal the bath

i) Using 10cc syringe and blunt tip, fill 


\section{5) Microiontophoresis and Micromanipulation}

a) Make and filter PSS solution ahead of time

i) $\left(119 \mathrm{mM} \mathrm{NaCl}, 25 \mathrm{mM} \mathrm{NaHCO}_{3}, 6 \mathrm{mM} \mathrm{KCl}\right.$, and $\left.3.6 \mathrm{mM} \mathrm{CaCl}_{2}\right)$

ii) $\mathrm{CaCl}_{2}$ must be added at the time of experiment or will fall out of solution

iii) Bubble with $5 \% \mathrm{CO}_{2} 90 \% \mathrm{~N}_{2}$ for 3 minutes prior to start of experiment

(1) Continue bubbling throughout experiment

b) Prepare glass micropipettes according to instructions using Dr. Nurkiewicz's electronic pipette puller and grinder.

i) Should have a $\sim 1 \mu \mathrm{m}$ opening.

ii) Make 1M ACh solution dissolved in double distilled water

iii) Use micro capillary needle to backfill, ensure no air in tip (should be able to see a tiny bubble of solution form on the tip of the pipette)

iv) Prepare at least 2 of these pipettes per animal, more if still learning.

c) Secure micropipette in micromanipulator

d) Insert platinum electrode wire in the blunt end

e) Place lead wire from the current programmer into the PSS bath and turn on holding current ( 200nA for 1M ACh)

i) Fault alarm should sound, gently place pipette tip in the PSS, if alarm stops, a circuit is achieved and it is good to proceed forward. If alarm doesn't stop, begin troubleshooting. 
f) Raise objective until it is at the very surface of the PSS. The more room between the objective and muscle the better at this point.

i) Using micromanipulator controls, carefully insert pipette tip into the bath and under the objective

(a) 45 degree angle

ii) At this point, the pipette tip is probably both not directly under the objective and not in focus, so it has to be found

(1) First step is to get the tip in the field of view of the objective. This is achieved by slowly sweeping the tip under the objective while looking through the eyepiece. A faint "shadow" should appear when the tip is in the field of view. This may take practice to be able to identify the shadow. (2) Once the tip is in the field of view, adjust focus until the tip is resolved. (3) Simultaneously lower tip and objective, keeping everything in focus, until the tip is at the surface of the muscle.

g) Carefully place the micropipette tip adjacent to the target vessel

i) Leave a few micrometers distance to prevent irritating the vessel when it dilates or spasms.

ii) There may be invisible connective tissue making it difficult to place the tip exactly where it is wanted

(1) Sometimes it is possible to work the tip through the tissue, other times it will need to be backed out and placed somewhere else

h) At the end of the 20min equilibration period, start running curves 
i) Raise the ejection current to achieve the desired total current and hold for 5 minutes.

(1) (Holding current - ejection current $=$ total current)

(2) i.e. $20,40,80,120 \mathrm{nA}$ seems to work well for ACh and SNP

(3) Apply in random order.

(a) Some solutes (SNP) have opposite polarity and currents will need to be reversed.

(4) Take 3 images at each time point over the course of a minute and average to account for any minor fluctuations in vessel diameter (a) If vessel is spasming, wait.

(5) 5 minute washout period following each ejection period.

(6) Following final washout period add $30 \mathrm{~mL}$ syringe of PSS plus $10^{\wedge}-4 \mathrm{M}$ adenosine to line. (In place of large bottle).

i) Analyze saved images to determine vessel diameter

i) Use equations provided in chapters 4 and 5

ii) 300 pixels $=100 \mathrm{uM}$ 


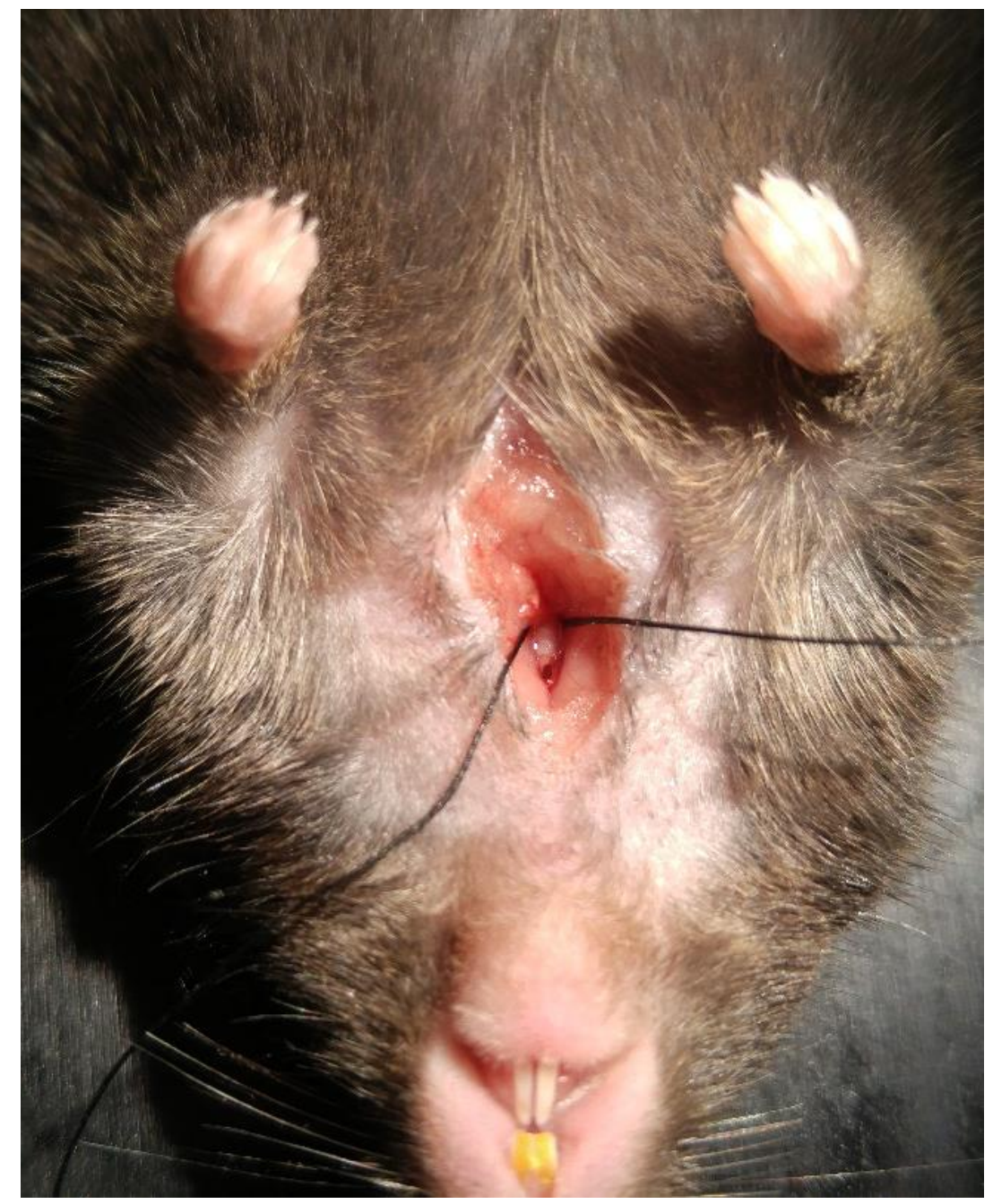

Figure 3.1 Mouse Tracheostomy. View of tracheostomy before placement of PE90 tubing. Note the placement of suture wire behind the trachea, between the muscle sheath. 


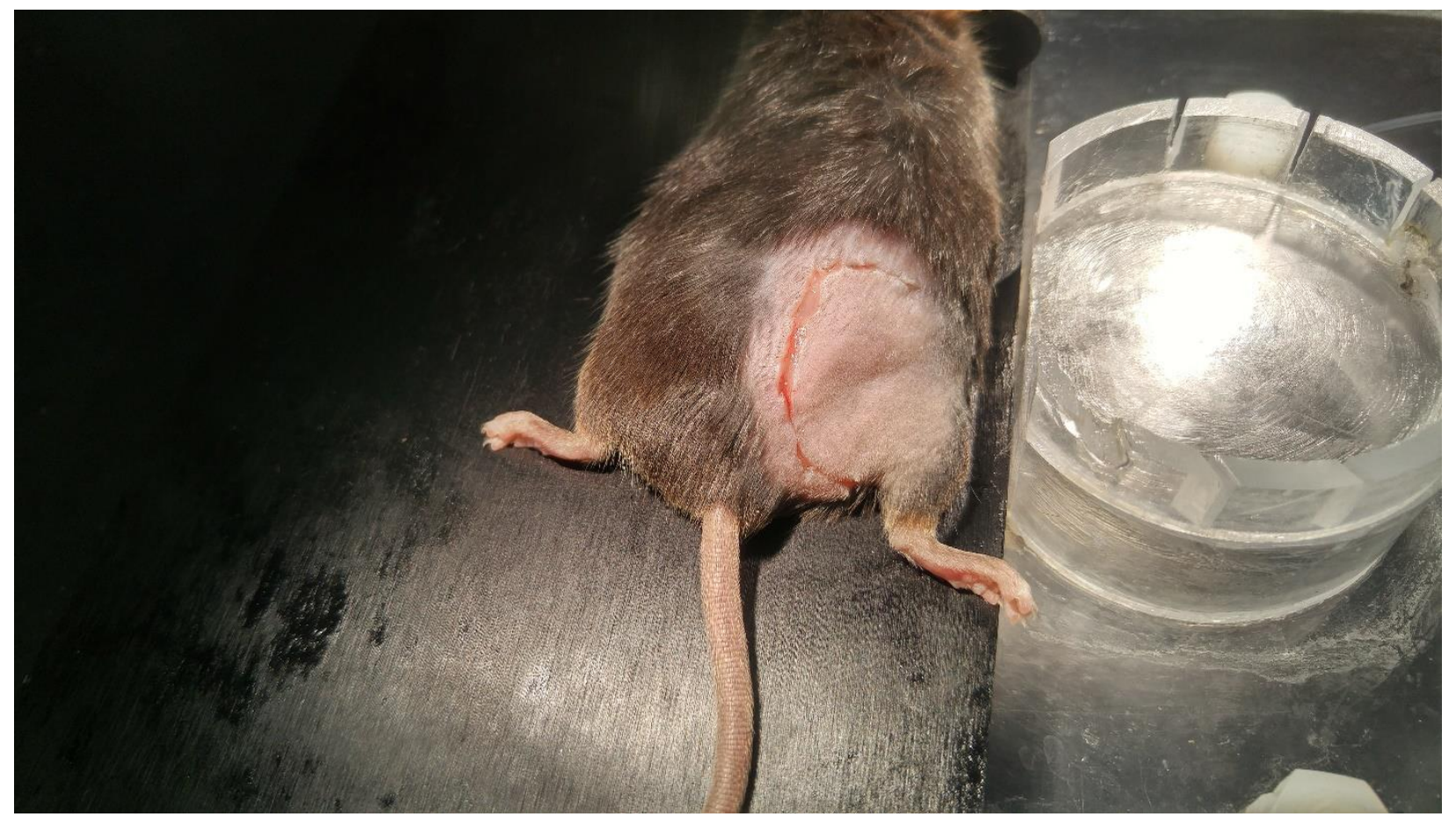

Figure 3.2. Skin incisions to expose the right gluteus maximus. 


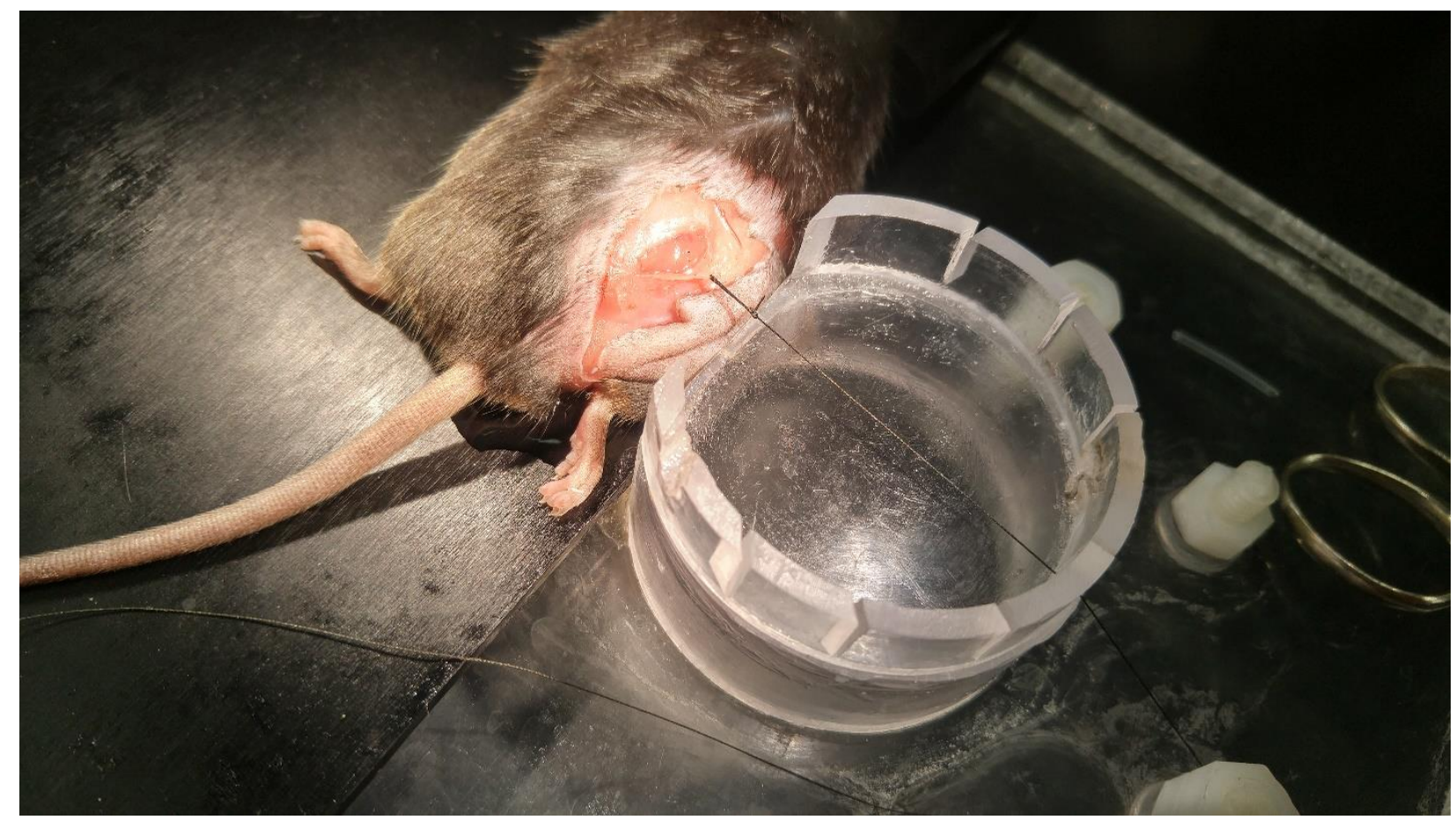

Figure 3.3. Externalization and fixation of the right gluteus maximus. The first suture is tied in at the medial apex of the externalized muscle flap. 


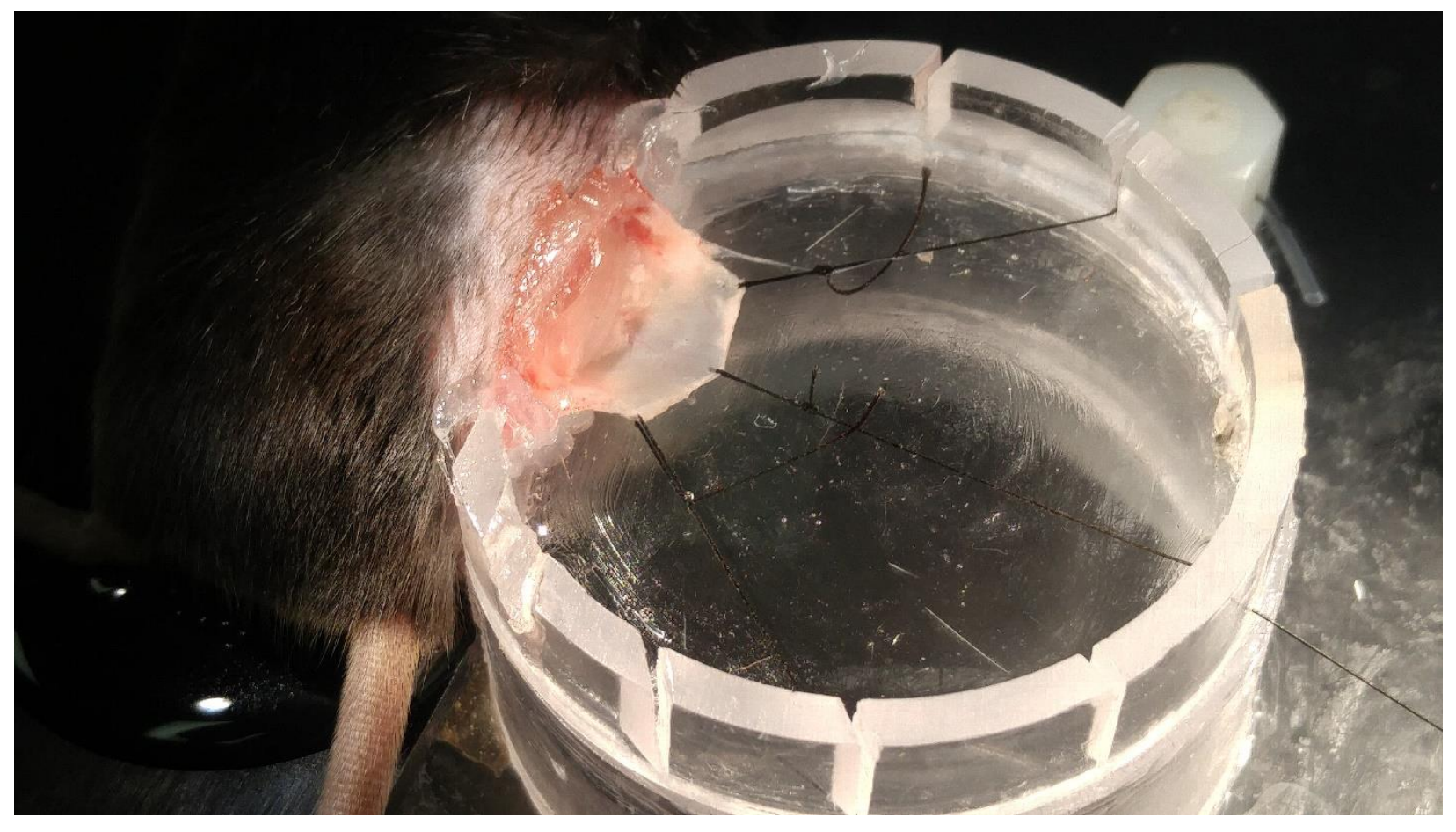

Figure 3.4. Fully externalized right gluteus maximus. Three suture wires secure the muscle flap in a PSS bath which is sealed with stopcock grease. 


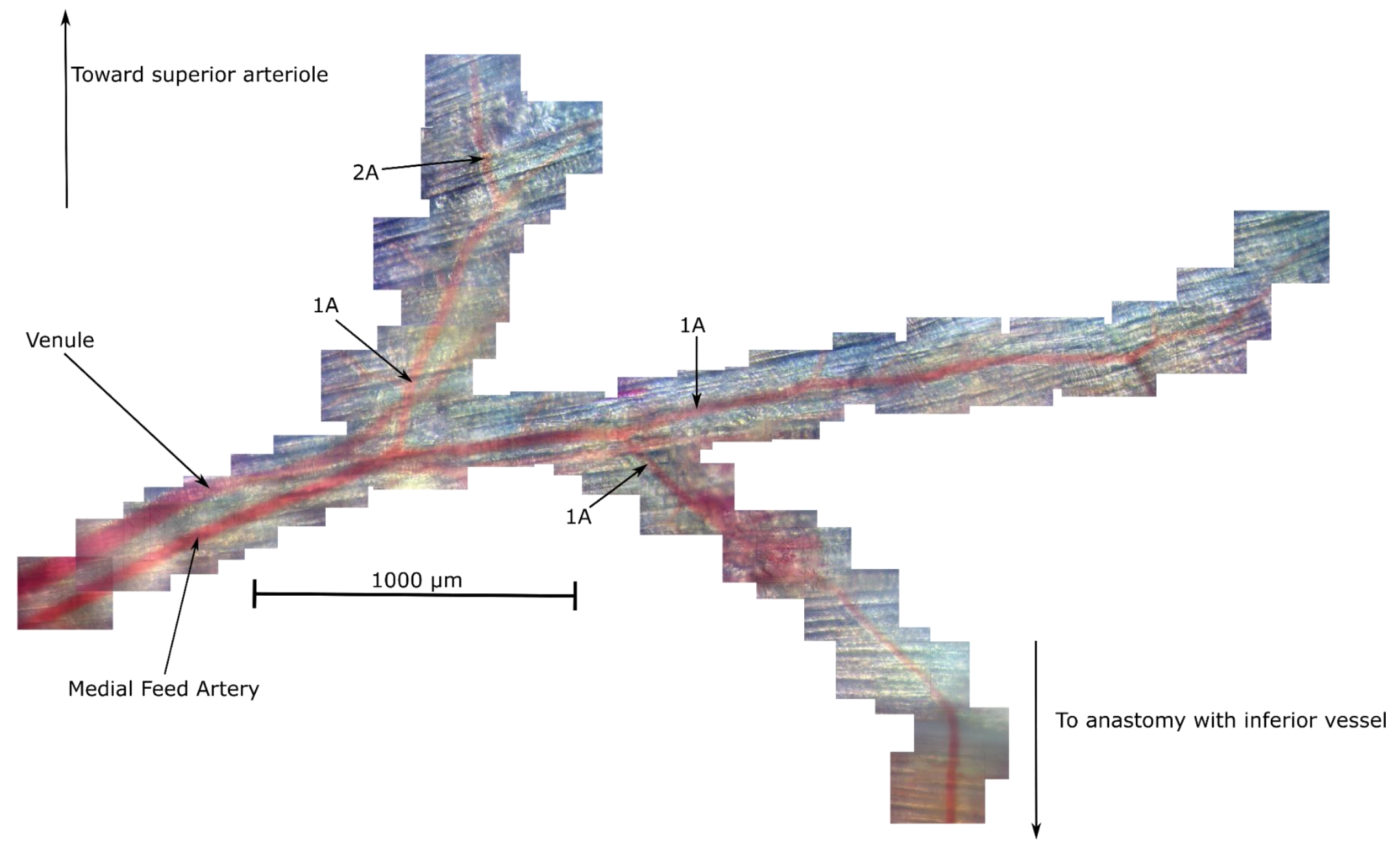

Figure 3.5. Representative image of medial arteriolar tree. Target $1 \mathrm{~A}$ vessels for iontophoretic stimulation. 


\section{Preliminary Results}

Arteriolar responses to acetylcholine, and phenylephrine were assessed in skeletal muscle arterioles of global TSP-1 knockout (KO) and wildtype (WT) littermate mice using IVM of the gluteus maximus. KO animals demonstrated $10.7 \%, 18.6 \%$, $16.5 \%$ greater vasodilatory response to acetylcholine concentrations of $10^{-6}, 10^{-4}$, and $10^{-2} \mathrm{M}(\mathrm{p}<0.05)$ (Fig 3.6). WT vessels demonstrated $23.8 \%$ greater constriction than $\mathrm{KO}$ at the highest concentration of phenylephrine, $10^{-3} \mathrm{M}(\mathrm{p}<0.05)$ (Fig. 3.7).

The results of this preliminary work established that our TSP-1 KO animals indeed exhibited phenotypically different arteriolar reactivities from WT controls, and that we were capable of reliably manipulating this system, thus establishing the groundwork for Aims 2 and 3. 


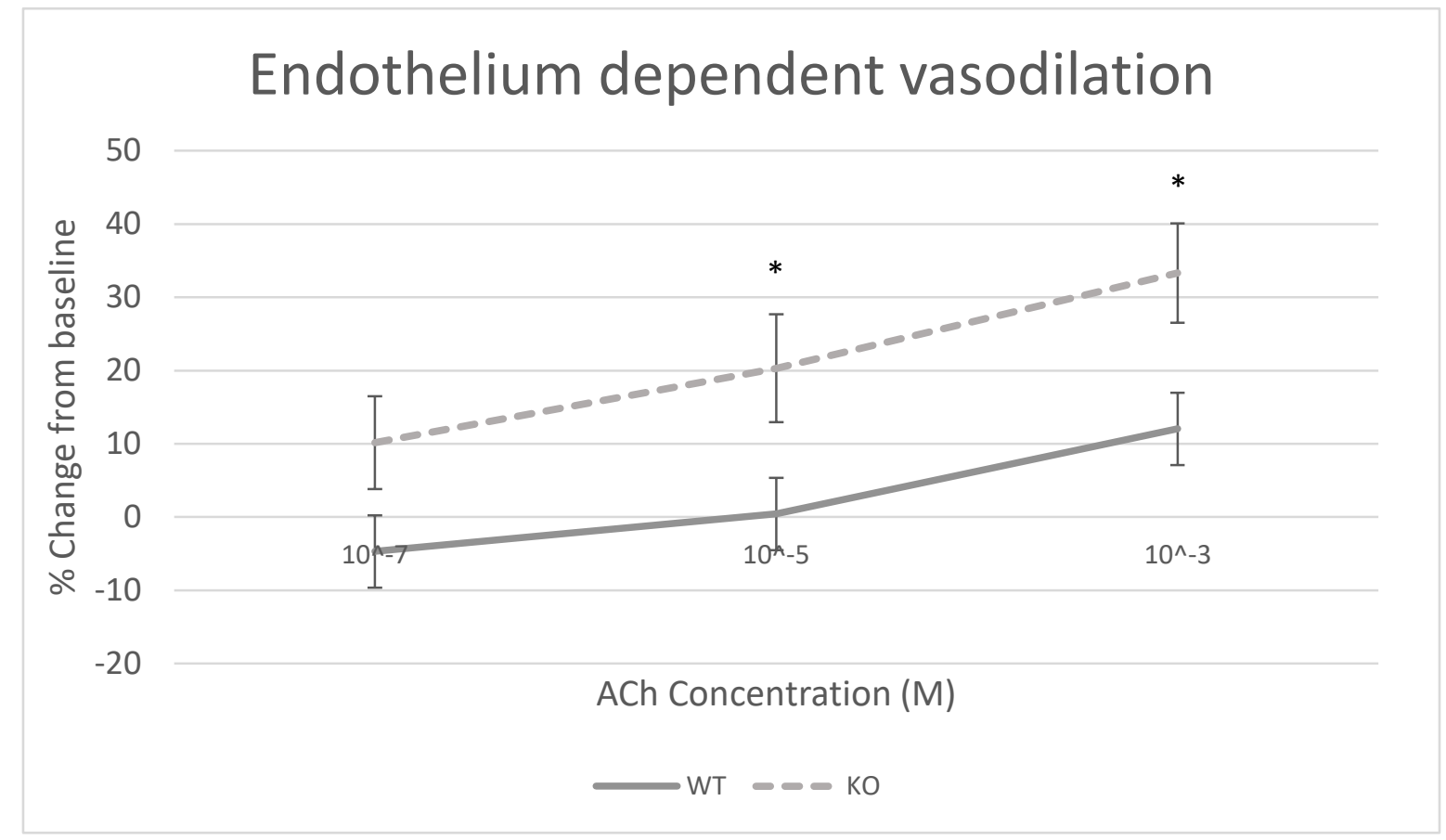

Figure 3.6. Endothelium dependent vasodilation. Arteriolar \% change from baseline following administration of acetylcholine (ACh) from wildtype (WT, $n=13)$ and thrombospondin-1 KO $(K O, n=13)$ groups. $(*)$ indicates difference between genotype. Values are mean $\pm S E . n$ represents number of vessels studied. 


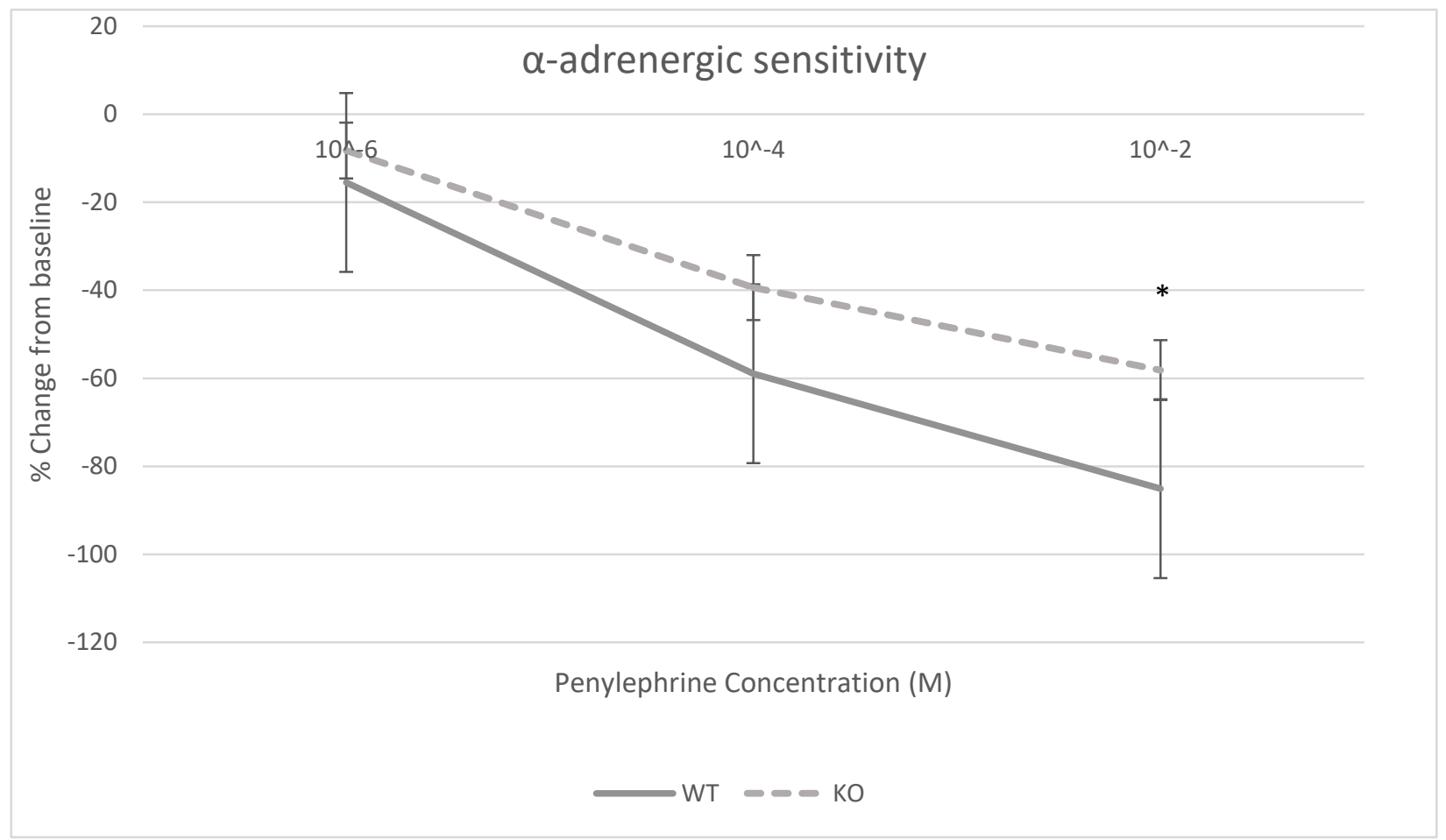

Figure 3.7. $\alpha$-adrenergic sensitivity. Arteriolar $\%$ change from baseline following administration of phenylephrine (PE) from wildtype $(\mathrm{WT}, \mathrm{n}=6)$ and thrombospondin-1 KO (KO, $\mathrm{n}=8$.) groups. $\left({ }^{*}\right)$ indicates difference between genotype. Values are mean \pm SE. $n$ represents number of vessels studied. 


\section{References}

Bauer, P.M., Bauer, E.M., Rogers, N.M., Yao, M.Y., Feijoo-Cuaresma, M., Pilewski, J.M., Champion, H.C., Zuckerbraun, B.S., Calzada, M.J. \& Isenberg, J.S., 2012. Activated CD47 Promotes Pulmonary Arterial Hypertension Through Targeting Caveolin-1. Cardiovascular Research, 93, 682-693.

Bearden, S.E., Payne, G.W., Chisty, A. \& Segal, S.S., 2004. Arteriolar Network Architecture And Vasomotor Function With Ageing In Mouse Gluteus Maximus Muscle. Journal Of Physiology-London, 561, 535-545.

Bearden, S.E. \& Segal, S.S., 2004. Motor Nerve Distribution Determines Feed Artery Control: Evidence From Mouse Gluteus Maximus Muscle. FASEB Journal, 18, Abst. 437.4-Abst. 437.4.

Csanyi, G., Yao, M., Rodriguez, A.I., Al Ghouleh, I., Sharifi-Sanjani, M., Frazziano, G., Huang, X., Kelley, E.E., Isenberg, J.S. \& Pagano, P.J., 2012. Thrombospondin-1 Regulates Blood Flow Via CD47 Receptor Mediated Activation Of NADPH Oxidase 1. Arteriosclerosis Thrombosis And Vascular Biology, 32, 2966-+.

Isenberg, J.S., Ridnour, L.A., Dimitry, J., Frazier, W.A., Wink, D.A. \& Roberts, D.D., 2006a. CD47 Is Necessary For Inhibition Of Nitric Oxide-Stimulated Vascular Cell Responses By Thrombospondin-1. Journal Of Biological Chemistry, 281, 2606926080.

Isenberg, J.S., Wink, D.A. \& Roberts, D.D., 2006b. Thrombospondin-1 Antagonizes Nitric Oxide-Stimulated Vascular Smooth Muscle Cell Responses. Cardiovascular Research, 71, 785-793.

Knuckles, T.L., Stapleton, P.A., Minarchick, V.C., Esch, L., Mccawley, M., Hendryx, M. \& Nurkiewicz, T.R., 2013. Air Pollution Particulate Matter Collected From An Appalachian Mountaintop Mining Site Induces Microvascular Dysfunction. Microcirculation, 20, 158-169.

Knuckles, T.L., Yi, J.H., Frazer, D.G., Leonard, H.D., Chen, B.T., Castranova, V. \& Nurkiewicz, T.R., 2012. Nanoparticle Inhalation Alters Systemic Arteriolar Vasoreactivity Through Sympathetic And Cyclooxygenase-Mediated Pathways. Nanotoxicology, 6, 724-735.

Minarchick, V.C., Stapleton, P.A., Sabolsky, E.M. \& Nurkiewicz, T.R., 2015. Cerium Dioxide Nanoparticle Exposure Improves Microvascular Dysfunction And Reduces Oxidative Stress In Spontaneously Hypertensive Rats. Frontiers In Physiology, 6, 12.

Moore, A.W., Bearden, S.E. \& Segal, S.S., 2010. Regional Activation Of Rapid Onset Vasodilatation In Mouse Skeletal Muscle: Regulation Through AlphaAdrenoreceptors. Journal Of Physiology-London, 588, 3321-3331.

Nurkiewicz, T.R., Porter, D.W., Hubbs, A.F., Stone, S., Chen, B.T., Frazer, D.G., Boegehold, M.A. \& Castranova, V., 2009. Pulmonary Nanoparticle Exposure Disrupts Systemic Microvascular Nitric Oxide Signaling. Toxicological Sciences, 110, 191-203. 
Sinkler, S.Y. \& Segal, S.S., 2014. Aging Alters Reactivity Of Microvascular Resistance Networks In Mouse Gluteus Maximus Muscle. American Journal Of PhysiologyHeart And Circulatory Physiology, 307, H830-H839.

Stapleton, P.A., Mcbride, C.R., Yi, J. \& Nurkiewicz, T.R., 2015. Uterine Microvascular Sensitivity To Nanomaterial Inhalation: An In Vivo Assessment. Toxicology And Applied Pharmacology, 288, 420-428. 


\section{Chapter 4: Thrombospondin-1 mediates Multi-Walled Carbon Nanotube induced impairment of arteriolar dilation.}

W. Kyle Mandler ${ }^{1}$, Timothy R. Nurkiewicz ${ }^{2,3}$, Dale W. Porter ${ }^{4}$, I. Mark Olfert ${ }^{1,3}$

${ }^{1}$ Division of Exercise Physiology, West Virginia University School of Medicine, Morgantown, WV 26506

2Department of Physiology and Pharmacology, West Virginia University School of Medicine, Morgantown, WV 26506

${ }^{3}$ Center for Cardiovascular \& Respiratory Sciences, West Virginia University, Robert C. Byrd Health Sciences Center, Morgantown, WV 26506

${ }^{4}$ National Institute for Occupational Safety and Health, Morgantown, WV 26505

Word count: 5,026

Short title: TSP-1, Multi Walled Carbon Nanotubes, and endothelial dysfunction

Corresponding Author:

Mark Olfert, PhD, FAHA

West Virginia University School of Medicine

One Medical Center Dr.

PO BOX 9227

Morgantown, WV 26506

304-293-7597

imolfert@hsc.wvu.edu

Disclaimer: The findings and conclusions in this report are those of the authors and do not necessarily represent the views of the National Institute for Occupational Safety and Health. Mention of any company name or product does not constitute endorsement by NIOSH. 


\section{Abstract}

Pulmonary exposure to multi-walled carbon nanotubes (MWCNT) has been shown to disrupt endothelium-dependent arteriolar dilation in the peripheral microcirculation. The molecular mechanisms behind these arteriolar disruptions have yet to be fully elucidated. The secreted matricellular matrix protein thrombospondin-1 (TSP-1) is capable of moderating arteriolar vasodilation by inhibiting soluble guanylate cyclase activity. We hypothesized that TSP-1 may be a link between nanomaterial exposure and observed peripheral microvascular dysfunction. To test this hypothesis, wild-type C57B6] (WT) and TSP-1 knockout (KO) mice were exposed via lung aspiration to $50 \mu \mathrm{g}$ MWCNT or a sham dispersion medium control. Following exposure (24hrs), arteriolar characteristics and reactivity were measured in the gluteus maximus muscle using intravital microscopy (IVM) coupled with microiontophoretic delivery of acetylcholine (ACh) or sodium nitroprusside (SNP). In WT mice exposed to MWCNT, skeletal muscle TSP-1 protein increased $>5$-fold compared to sham exposed, and exhibited a $39 \%$ and $47 \%$ decrease in endothelium-dependent and independent vasodilation, respectively. In contrast, TSP-1 protein was not increased following MWCNT exposure in KO mice and exhibited no loss in dilatory capacity. Microvascular leukocyte leukocyte-endothelium interactions were measured by leukocyte adhesion and rolling activity in assessing third order venules. The WT+MWCNT group demonstrated $223 \%$ higher leukocyte rolling compared to WT+SHAM controls. TSP-1 KO animals exposed to MWCNT showed no differences from WT+SHAM control. These data provide evidence that TSP-1 is likely a 
central mediator of the systemic microvascular dysfunction that follows pulmonary MWCNT exposure.

Keywords

Inflammation, endothelial dysfunction, microvascular dysfunction, nitric oxide 


\section{Introduction}

Anthropogenic materials designed and manufactured to take advantage of unique properties displayed at the nanoscale (less than $100 \mathrm{~nm}$ in one dimension) are known as engineered nanomaterials (ENM) (Borm and Mueller-Schulte, 2006). With applications ranging from aerospace to biomedicine, ENM are rapidly becoming an integral part of our global economy. It is becoming increasingly apparent that ENM exposure, especially via the lungs, can result in significant toxicities in both the pulmonary and cardiovascular systems. Because of their growing ubiquitous commercial and industrial use and wide ranges of ENM characteristics (e.g. size, shape, redox status, surface functionalization, and rate of dissolution), there is critical need to better understand the biological responses of ENM in mammals. At present, however, the molecular signaling events underpinning cardiovascular ENM toxicity are not completely understood.

Of particular concern are ENM-mediated changes in microvascular function, specifically the ability of the arterioles to maintain appropriate resistance and reactivity through paracrine signaling from the vascular endothelium. The arterioles, in contrast to larger conduit and compliance vessels, are particularly important in blood flow regulation as they are the principal source of active peripheral resistance in the systemic vasculature. There is increasing evidence that exposure to ENM, especially via pulmonary routes, can result in disruption of normal arteriolar function. In vitro studies have demonstrated changes in autophagic cell death (Yamawaki and Iwai, 2006, Stern et al., 2012, Zhang et al., 2016a) in human vascular endothelial cells following nanomaterial exposure. A significant pro-inflammatory shift (Gojova et al., 2007, Corbalan et al., 2011, Zhu et al., 
2011) in endothelial cells has also been noted, and may be the result of generation of reactive oxygen species (Liu and Sun, 2010). Reduced capacity for arteriolar (Stapleton et al., 2012a) endothelium-dependent vascular relaxation, as well as enhanced coronary arterial vasoconstriction (Thompson et al., 2014) following pulmonary exposure to multiwalled carbon nanotubes (MWCNT) has been documented. While oxidative stress has been observed in these studies, the underlying molecular mechanisms for many of these changes remain to be fully defined.

Arteriolar diameter is the result of a complex interplay between neural, endocrine, paracrine, and local metabolic factors. By responding to these inputs via changes in contractile activation of myosin and actin in the vascular smooth muscle cells, the arterioles contribute to the maintenance of homeostasis by altering resistance to match blood flow to local metabolic demand while simultaneously adapting to systemic pressure gradient changes. In this manner, adequate tissue perfusion is maintained and capillary damage due to excess pressure and flow is prevented. One of the most important factors in this regulatory system is the ability of the vascular endothelium to stimulate smooth muscle relaxation via nitric oxide (NO). The vascular endothelium is responsible for detecting changes in physical influences such as shear stress, as well as humoral influences including the neurotransmitters acetylcholine (ACh) and norepinephrine. These signals are transduced through activation of endothelial nitric oxide synthase (eNOS) via both calcium dependent and calcium independent pathways (Fleming et al., 1997). First discovered in the context of its role in wound healing, TSP-1 comprises the bulk of the protein found in the alpha granules of platelets (Baenzige.NI et 
al., 1971). Part of a larger six member thrombospondin family, TSP-1 is a $450 \mathrm{kD}$ homotrimeric glycoprotein shown to be an important regulator of multiple points in the NO signaling pathways. This control is achieved primarily through binding to cell surface receptor CD47 in the vascular smooth muscle, and limiting cGMP production (Isenberg et al., 2005a) by inhibiting sGC activity (Isenberg et al., 2006b). TSP-1/CD47 can also inhibit eNOS activity by altering $\mathrm{Ca}^{2+}$ transients in endothelial cells, blocking phosphorylation of eNOS at serine ${ }^{1177}$ (Bauer et al., 2010). Another receptor for TSP-1 more commonly associated with regulation of angiogenesis, CD36, may also play a role in inhibiting eNOS by displacing it from intracellular caveolae (Shaul, 2003). The TSP-1/CD47/CD36 signaling axis has been implicated as a contributing factor, or in the etiology, to a number of disease states, including coronary artery disease (Celermajer et al., 1992, Schachinger et al., 2000), obesity and metabolic syndrome (Steinberg et al., 1996, Varma et al., 2008, Matsuo et al., 2015), hypertension (Panza et al., 1990, Boulanger, 1999), peripheral arterial disease (Smadja et al., 2011), diabetes mellitus (Cai and Harrison, 2000), stroke (Cho, 2012) among others (Roberts et al., 2012, Kong et al., 2013).

Despite the apparent involvement of TSP-1 in influencing endothelial function and arteriolar reactivity, the effect of ENM exposure on its expression and activity and subsequent cardiovascular effects have yet to be investigated. In the current study, we hypothesize that pulmonary MWCNT exposure elevates peripheral expression of TSP-1 protein, mediating systemic arteriolar endothelial dysfunction; and that mice lacking the TSP-1 gene (i.e. knockout mice) will be protected from this effect due to the lack of TSP1 negative influence on endothelial NO production. 


\section{Methods}

\section{Animals}

C57BL/6] background TSP-1 knockout (TSP-1 KO) and C57BL/6] animals (WT) were purchased from Jackson Labs, Bar Harbor, ME (Stock\# 006141 and 000644, respectively) and used to develop an in house breeding colony. Mice were housed according to NIH Use of Laboratory Animals, Animal Welfare Act, and all experiments were approved by the West Virginia University Institutional Animal Care and Use Committee. Mice were kept on a 12 hour light/dark cycle, and provided food and tap water ad libitum. Experiments were scheduled so that data collection occurred at approximately 16 weeks of age. At this age, the mice are sexually and developmentally mature and the gluteus maximus muscle is of sufficient size and strength to undergo intravital microscopy (Flurkey et al., 2007). In order to reduce the number of waste animals generated, both male and female WT and KO animals were used in all experiments in approximately equal numbers (Table 1). We have observed no significant sex differences in vessel baseline diameter, maximal passive diameter, or reactivity before and after nanomaterial exposure.

[Table 1 near here]

\section{Nanomaterial exposure details}

MWCNT are catalytically grown by chemical vapor deposition processes and were obtained from Mitsui \& Co (MWNT-7, Lot \# 061220-31, Ibaraki, Japan). Mitsui-7 MWCNTs were designated as the ENM of choice for this project based on the wide number of 
potential applications, observed toxicity, and prior investigations by our group (See below for physico-chemical details) Oropharyngeal aspiration, as opposed to intratracheal instillation or aerosol inhalation was chosen as the method of exposure for this study due to the high degree of flexibility and scalability compared to inhalation, and is less traumatic for the animals than intratracheal instillation (Rao et al., 2003). Aspiration of this dose of MWCNT in dispersion medium generates pulmonary response analogous to instillation (Bonner et al., 2013) and inhalation (Shvedova et al., 2008, Mercer et al., 2011). Similar patterns of altered systemic vascular reactivity and NO signaling were observed in this study when compared to similar measurements following inhalation of aerosolized MWCNT (Nurkiewicz et al., 2009). While anesthetized with isoflurane, solution of $50 \mu \mathrm{g}$ MWCNT suspended and dispersed using ultrasonication in a phospho-buffered saline/purified mouse serum albumin (PBS/MSA) medium was placed in oropharynx with the tongue manually extended. Upon release, the tongue retracts and the mouse reflexively inhales, aspirating the material and depositing in the lung(Rao et al., 2003). This dose of MWCNT has been shown to cause lung injury and inflammation using the same exposure method as used in this study (Porter et al., 2010). Control animals received a sham aspiration of medium without nanomaterials. This dispersion medium (DM) has been shown to be both biocompatible, no immunogenic, and effective at dispersing MWCNT (Porter et al., 2008, Porter et al., 2010). 


\section{Nanomaterial Physico-chemical properties}

Extensive characterization of the MWCNT has been previously reported (Porter et al., 2010). In summary, the overall MWCNT trace metal contamination was determined to be $0.78 \%$, with sodium and iron being the major metal contaminants and is free of endotoxin. Because of the presence of iron, reactive oxygen species generation from MWCNT was investigated using electron spin resonance (ESR) spectroscopy. No hydroxyl radical generation was detected from MWCNT in acellular ESR experiments, indicating the iron present in MWCNT was not capable of generation reactive oxygen species. X-ray photoelectron spectroscopy measurements indicated the formation of the hydroxyl groups on the nanotube surface, which was consistent with the $-11 \mathrm{mV}$ zeta potential of the MWCNT in DM. High resolution transmission electron microscopy determined the number of walls ranged from 20 to 50 . MWCNT width distribution followed a normal distribution with count mean width of $49 \pm 13.4$ (S.D.) nm, while MWCNT length distribution was log normal with median length of $3.86 \mu$ m (GSD 1.94).

\section{Intravital microscopy and iontophoresis of mouse gluteus maximus arterioles}

[Figure 1 near here.]

At 24-h post-aspiration, KO or WT mice were anesthetized using thiobutabarbital (Inactin, $100 \mathrm{mg} / \mathrm{kg}$, i.p.) and placed on a thermo-probe regulated heating pad to maintain a $37^{\circ} \mathrm{C}$ core temperature. The trachea was intubated to ensure a patent airway. The right gluteus maximus muscle was surgically exteriorized for microscopic observation, leaving its innervation and all feed vessels intact. This site of observation was chosen as 
skeletal muscle is the largest global reservoir of the microvasculature and the largest source of active peripheral resistance. After exteriorization, the muscle was secured over an optical pedestal with 5-0 suture wire at its in situ length. The pedestal was sealed with grease to create a superfusate bath. Throughout the surgery and following experimental period, the muscle was continuously superfused with an electrolyte solution (119 mM $\mathrm{NaCl}, 25 \mathrm{mM} \mathrm{NaHCO}_{3}, 6 \mathrm{mM} \mathrm{KCl}$, and $3.6 \mathrm{mM} \mathrm{CaCl} 2$ ), warmed to $35^{\circ} \mathrm{C}$, and equilibrated with $95 \% \mathrm{~N}_{2}-5 \% \mathrm{CO}_{2}(\mathrm{pH}=7.35-7.40)$. Superfusate flow rate was maintained at 4 $\mathrm{ml} / \mathrm{min}$ to minimize equilibration with atmospheric oxygen.

The animals were transferred to an intravital microscope coupled to a digital video camera. Observations were made with a 20X water immersion objective. A micropipette with an opening of $1-2 \mu \mathrm{m}$ was filled with a $10^{-2} \mathrm{M}$ acetylcholine (to measure endothelium dependent vasodilation) or sodium nitroprusside (to measure endothelium independent vasodilation) solutions. The tip of the pipette was guided under microscope to a point adjacent to the adventitia of the target arteriole. During this time a holding current of $200 \mathrm{nA}$ or $200 \mathrm{nA}$, respectively, was applied to the bath to prevent ACh/SNP leakage from the pipette. Once in place and following a 20-minute equilibration period, ejection currents of $20 n A, 40 n A$ and $80 n A$, and $120 n A$ (for ACh) or $-20 n A,-40 n A$ and $-80 n A$, and -120 nA (for SNP) were applied to the bath for 5 minutes each in a randomized order, with a 5-minute washout period between each step. Following the final ACh/SNP washout period, adenosine was added to the superfusate at a concentration of $10^{-2} \mathrm{M}$ to determine vessel maximal passive diameter. Images were displayed on a high-resolution computer monitor and digitally captured in the last minute of each step for later analysis using 
Image] (Image] 1.46v, National Institutes of Health). One to three first order arterioles were studied per mouse. Measurements of vessel diameter were made from recorded images. Vessel reactivity is reported as percent of maximal diameter achieved. Resting vascular tone was calculated for each vessel as follows:tone $=\left[\frac{D \text { pass }-D c}{D \text { pass }}\right] \times 100$, where $D$ is arteriolar diameter, in micrometers, and $D_{\text {pass }}$ is passive diameter under $A D O$, and $D_{c}$ is the diameter measured during the control period. $D_{c}$ was taken following a 20 -minute equilibration and only if no change in vessel diameter had been observed for at least 1 minute prior. Percent relaxation was calculated at each ACh or SNP ejection current as follows: \% relaxation $=\left[\frac{\text { Dpass }- \text { Dion }}{\text { Dpass }-D C}\right] \times 100$, where $D_{\text {ion }}$ is the vessel diameter following 5 minutes of drug delivery via iontophoresis.

\section{Leukocyte adhesion and rolling}

Prior to the start of iontophoresis, venules three branch orders from the capillaries, approximately $50 \mu \mathrm{m}$ in diameter, and within $100 \mu \mathrm{m}$ of target arterioles (typically the paired venule) were identified and video recordings made. Adhering or rolling leukocytes were quantified to characterize microvascular inflammation. Leukocytes that were either stationary or moving but in constant contact with the venular wall for at least $200 \mu \mathrm{m}$ were counted for $1 \mathrm{~min}$ in each venule studied. Following the completion of iontophoresis studies and after a 20 minute washout period, $10^{-4} \mathrm{M}$ apocynin was added to the superfusate to examine contributions of NADPH oxidase generated reactive oxygen species. Following a 30 minute apocynin incubation period, the measurements were repeated. 


\section{$\underline{\text { TSP-1 tissue protein }}$}

Following the IVM procedure, animals were sacrificed and triceps surae muscles (gastrocnemius, soleus, and plantaris) were harvested and immediately snap frozen in liquid nitrogen. Homogenates were centrifuged at $4{ }^{\circ} \mathrm{C}$, at $8,000 \mathrm{~g}$ for 10 minutes, and supernatants removed and placed in new tubes. Total protein was measured by Bradford assay (\#23236 Pierce Coomassie Plus Protein Assay Kit, Thermo Scientific, Rockford, IL, USA). Muscle samples were denatured (36 $\mu \mathrm{g} \mathrm{GA}$ ) and separated on 26-well 8\% Bis-Tris Midi SDS-PAGE Gels (Novex WG1003BX10, Carlsbad, CA, USA) for 45 min at $120 \mathrm{~V}$ and blotted onto a $0.45 \mu \mathrm{m}$ nitrocellulose membrane (nitrocellulose membrane no. 88018, Thermo Scientific, Rockford, IL, USA) for 90 min at $50 \mathrm{~V}$. Total lane protein was determined using Ponceau S stain (0.1\% (w/v) in 5\% acetic acid, P7170 Sigma-Alrdrich, St. Louis, MO). The membrane was blocked with $5 \%$ fat free milk in Tris buffered saline and probed with antibodies: TSP-1 (1:500, A6.1, sc-59887, Santa Cruz Biotechnology, Santa Cruz, CA, USA). Chemiluminescence detection allowed for visualization of proteins (Pierce ECL Western Blotting Substrate, no. 32209, Rockford, IL, USA), images of blots were taken using Genesnap software (version 7.01; Syngene, Frederick, MD, USA) after 25 min exposure. Densitometry was measured using Image Studio Lite Version 5.2 (LiCor Biosciences, Lincoln, NE, USA). Values are reported as target protein densitometry normalized to total lane protein. 


\section{Statistics and calculations}

A D'Agostino \& Pearson omnibus normality test was used to assess homogeneity of variance. In cases where non-normal datasets were detected, statistical comparisons were made with Kruskal-Wallis tests and post-hoc Wilcoxon rank-sum tests for between subject effects where appropriate. A $2 \times 2$ factorial ANOVA was performed to compare the main effects of exposure (sham vs MWCNT) and genotype (WT vs KO) for each variable. When significant main effects were observed a Sidak post-hoc test was used to determine where significant differences existed between the groups. In all cases, $p<$ 0.05 was used to establish statistical significance. All data are reported as mean $\pm \mathrm{SEM}$, unless otherwise noted.

\section{Results}

\section{$\underline{\text { TSP-1 Skeletal Muscle Protein }}$}

[Figure 2 near here] TSP-1 protein was assessed in skeletal muscle 24 hrs. postaspiration. TSP-1 protein increased by $512 \pm 21 \%$ in the WT+MWCNT group compared to sham controls (Fig. 2). TSP-1 protein in both TSP-1 KO groups was significantly lower than control and did not change with treatment. The interaction of Exposure and Genotype was significant $(F(1,19)=8.5, p=0.009)$.

\section{Baseline and maximal passive diameter}


There were no differences between any groups or genders in baseline vessel diameter, maximal passive diameter, or spontaneous vessel tone (Table 2). [Table 2 near here]

\section{Endothelium-dependent vasodilation}

Significant differences in absolute arteriole diameter were noted between groups with both ACh and SNP stimulation. (Fig. 3). [Figure 3 near here]. However, to normalize for variation in baseline diameter, \% relaxation will be used henceforth as a measure of arteriolar reactivity (Fig. 3). Full vessel diameter curves for each group are presented in supplemental figures 1 and 2. At 120 nA ACh ejection current, TSP-1 KO+SHAM animals demonstrated enhanced vasodilatory capacity compared to WT+SHAM $(125 \pm 19 \%$ vs $90 \pm 3 \%)$. Enhanced dilatory capacity was maintained in the TSP-1 KO + MWCNT exposure (121 $\pm 8 \%)$. In contrast, WT+MWCNT animals exhibited a significantly blunted response to ACh compared to control and TSP-1 KO $(61 \pm 9 \%)$. A similar response was noted at 80 nA ejection current; the KO+SHAM group was able to dilate to a greater extent than WT+SHAM $(97 \pm 6 \%$ vs $56 \pm 9 \%)$. Dilatory capacity was again maintained in the KO+MWCNT group $(110 \pm 7 \%)$ while the WT+MWCNT group was limited to $39 \pm 14 \%$. At $40 \mathrm{nA}$, both $\mathrm{KO}+\mathrm{SHAM}$ and $\mathrm{KO}+\mathrm{MWCNT}$ groups were able to dilate more than WT+MWCNT (45 $\pm 16 \%, 54 \pm 9 \%$, respectively vs $15 \pm 11 \%)$. The KO+MWCNT was able to dilate greater than WT+SHAM control $45 \pm 17 \%)$. No significant differences were observed at the $20 \mathrm{nA}$ acetylcholine ejection current level. There was no observed interaction between Ejection Current and Genotype $(F(15,141)=1.585, P=0.0850)$ Area under the curve for each group is as follows in units of $\%$ dilation $x$ ejection current; 
WT+SHAM， 5777 $\pm 215 ，$ WT+MWCNT， 3224 $\pm 452.5 * ， K O+S H A M *+， 7698 \pm 213 ，$ KO+MWCNT $8584 \pm 483 * \dagger .\left(^{*}\right)$ indicates difference from WT+SHAM, $\left({ }^{\dagger}\right)$ indicates difference from WT+MWCNT.

\section{Endothelium-independent vasodilation}

[Figure 4 near here]

When stimulated with the membrane dependent NO donor SNP, the WT Sham group and both TSP-1 KO groups were able to achieve similar levels of vasodilation at

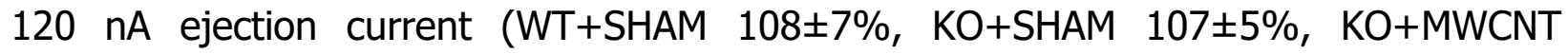

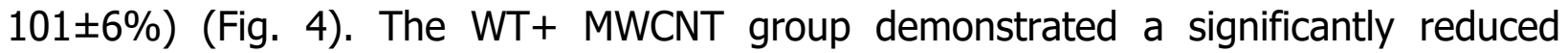
vasodilatory response compared to the other groups, (61士7\%). At the $80 \mathrm{nA}$ ejection current the KO+SHAM group demonstrated enhanced dilation compared to WT+MWCNT ( $108 \pm 7 \%$ vs. $66 \pm 7 \%)$. There was no observed interaction between Ejection Current and Genotype $(F(5,45)=1.230, P=0.3110)$ Area under the curve for each group is as follows in units of $\%$ dilation $x$ ejection current; WT+SHAM, 10478+ WT+MWCNT,

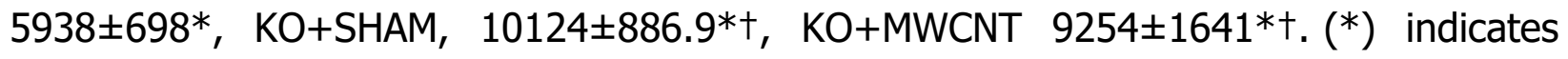
difference from WT+SHAM, $\left({ }^{\dagger}\right)$ indicates difference from WT+MWCNT.

\section{Leukocyte adhesion and rolling}

[Figure 5 near here]

A component of vascular inflammation, leukocyte adhesion was assessed by measuring leukocyte flux (CPM) through third order venules during intravital experiments (Fig. 5) For the WT+SHAM group, the mean flux was $79.3 \pm 6.9$ CPM. The WT+MWCNT 
group demonstrated an $81 \%$ increase in leukocyte flux compared to sham control $(176.7 \pm 13.5$ CPM). The KO+MWCNT did not demonstrate significantly increased leukocyte flux compared to the WT+SHAM group, but was lower than WT+MWCNT (100.3 $\pm 9.1 \mathrm{CPM})$. Following 30 minutes of incubation with $10^{-4} \mathrm{M}$ apocynin in the physiologic saline solution (PSS) superfusate, repeat measurements were made on the same vessels. All groups demonstrated significant reductions in leukocyte flux, compared

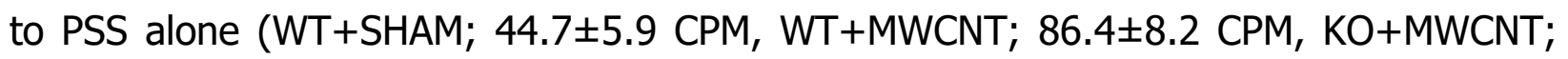
55.5 \pm 5.2 CPM). Both WT+SHAM and KO+MWCNT were lower than WT+MWCNT group following apocynin incubation. Apocynin decreased leukocyte flux in the WT+MWCNT group to a level similar to the WT+SHAM group with PSS alone.

\section{Discussion}

The key finding of the current study is the apparent involvement of TSP-1 in mediating pulmonary MWCNT exposure induced disruption in peripheral vascular function, as demonstrated by the protection offered to the TSP-1 KO group following MWCNT exposure.TSP-1 KO animals were able to achieve similar dilation after MWCNT exposure in response to both ACh and SNP stimulation compared to sham controls. These data represent the first evidence that TSP-1 may act as a key mediator in the microvascular dysfunction that follows MWCNT exposure. Intravital microscopy and iontophoresis in the mouse gluteus maximus microvasculature has been well established (Bearden and Segal, 2004, Jackson and Segal, 2006, Sinkler and Segal, 2014), and compared to preparations using isolated vessels, mesentery artery, hamster cheek pouch, 
or cremaster, the gluteus maximus preparation allows visualization of fully perfused and innervated microvasculature in locomotive skeletal muscle. The results of this MWCNT exposure were obtained by using a powerful technique for the assessment of microvascular function in vivo combined with a well-characterized KO model. This study also represents one of a handful of attempts to use $\mathrm{KO}$ animals to investigate molecular mechanisms of nanotoxicity (Bagher et al., 2011b, Louro et al., 2014, Chen et al., 2015, Aragon et al., 2016), and is the first in the area of the peripheral microcirculation.

Our findings of impaired vasodilation agree with previously undertaken investigations of arteriolar reactivity following MWCNT as well as other ENM exposures. As a large endocrine, paracrine, and autocrine organ, the vascular endothelium is a smooth monolayer of cells lining interior vessel lumen and has a number of responsibilities aside from regulating vessel diameter, including balancing pro- and anti-inflammatory factors, regulating vascular permeability, and moderating leukocyte adhesion and rolling (Inagami et al., 1995). Stapleton et al. demonstrated significant inhibition of endothelium dependent dilation in response to increasing concentrations of ACh $\left(10^{-9} \mathrm{M}-10^{-4} \mathrm{M}\right) 24$ hrs. post MWCNT inhalation in isolated rat coronary arterioles. This inhibition was maintained out as far as 168 hours post-exposure. Impaired responsiveness to the calcium ionophore A23187 was also observed at 24 hours, and persisted to a lesser degree at longer time points (Stapleton et al., 2012a). In a separate study, this group has also presented evidence that lung nanomaterial exposure generates peripheral microvascular ROS stress and decreased NO bioavailability (Nurkiewicz et al., 2009). The authors reported that endothelium dependent vasodilation (via A23187 incubation) was 
inhibited, however vascular smooth muscle sensitivity to exogenous NO donation was not impaired. When vessels were incubated TEMPOL/catalase, apocynin, or ABAH, normal endothelial function was partially restored. In contrast, in our study when stimulated with SNP, all groups except for the WT+ MWCNT were able to achieve similar levels of dilation, indicating deficits in smooth muscle NO responsiveness. The discrepancy here might be explained by several differences between the studies, such as different species and microvascular bed. Keeping in mind our observed concomitant increase in TSP-1 protein levels, it follows that the WT+MWCNT would still exhibit blunted endotheliumindependent vasodilation, as TSP-1 primarily acts to limit vasodilation by inhibiting the NO-sGC-cGMP pathway downstream of sGC at the cGMP-dependent kinase (Miller et al., 2010). We also observed that TSP-1 KO animals were able to achieve robust vasodilation in response to increasing doses of ACh. The role of the TSP-1/CD47 axis in mediating smooth muscle contractile activity has been extensively characterized (Isenberg et al., 2005b, Isenberg et al., 2006a, Isenberg et al., 2007a). Most relevant to the current study was work published in 2010 by Bauer et al. (Bauer et al., 2010). In this study, the authors report similar levels of relaxation ( $125 \%$ of maximal passive diameter) in response to ACh in TSP-1 null animal vessels. Furthermore, when exogenous TSP-1 was supplied, "normal" relaxation was restored. The authors observed a similar disruption in dilatory capacity in CD47 KO animals, however when exogenous TSP-1 was supplied, the impaired dilation remained, suggesting that CD47 receptor is an important pathway involved with these TSP-1 mediated effects. But it is likely that TSP-1 signaling following nanomaterial exposure is not limited to CD47. 
Recently, a role for CD36 has been described in mediating vascular dysfunction following MWCNT inhalation (Aragon et al., 2016). The authors report that endothelial cells incubated in serum collected from mice post exposure generated significantly less NO, independent of NO scavenging. Furthermore, isolated aortic rings incubated in exposed serum exhibited decreased vasodilatory responses to acetylcholine. When the same procedure was repeated on aortic rings isolated from CD36 KO animals, the impairment was abolished, suggesting that factors released in lungs exposed to MWCNT are able to exert peripheral effects. In this case, the authors report elevated matrix metallopeptidase-9 (MMP-9) as one potential culprit, as serum from MMP-9 KO mice induced a modest right-shift effect on the concentration response to ACh, but did not completely reduce the overall magnitude of relaxation.

While there is strong evidence for disruption of endothelium dependent vasodilation by direct action of TSP-1 on the NO signaling pathway, it is also possible that TSP-1 may also interfere with NO signaling through endothelium independent mechanisms. For example, activated leukocytes released from the lung adhering to and rolling on the venular walls is known to increase ROS activity (Stapleton et al., 2007). Our observation that leukocyte adhesion and rolling was enhanced in WT mice after pulmonary MWCNT exposure is in agreement with previous work with pulmonary exposure to other particulate matter (Nurkiewicz et al., 2004, Stapleton et al., 2015b). Our data represent the first demonstration that the loss of TSP-1 afforded protection to the microvasculature, as evidenced by reduced leukocyte rolling in TSP-1 KO compared to WT MWCNT exposed mice. At present, the molecular signaling responsible for the 
observed increase in skeletal muscle TSP-1 protein remains unclear, however we speculate that the pathway likely involves lung injury and innate immune response, leading to the activation of leukocytes. Evidence supporting this is robust as MWCNT are capable of being injurious and pro-inflammatory to the pulmonary epithelium (Porter et al., 2010, Porter et al., 2013) including increasing permeability (Thompson et al., 2016) and enhancing levels of plasma inflammatory cytokines (Crouzier et al., 2010). Indeed, TSP-1 is strongly expressed in leukocytes (Wight et al., 1985) and platelets (Brown and Frazier, 2001) which are activated the lung (Morimoto et al., 2013) and in the blood (Bihari et al., 2010) and form circulating conjugates following nanotube exposure-related lung injury (Nemmar et al., 2007). TSP-1 activation of its CD47 receptors is also well known as positive regulators of leukocyte-endothelium adhesion (Brown and Frazier, 2001, Narizhneva et al., 2005), stimulating expression of cell adhesion molecules ICAM1 and VCAM-1 (Narizhneva et al., 2005). Therefore, it is possible that TSP-1 may act to encourage leukocyte adhesion to the vessel wall, promoting NO quenching while simultaneously disrupting NO signaling through CD47 signaling.

The present study was focused on the acute time-frame to determine if TSP-1 was an important factor involved in peripheral vascular dysfunction following exposure to MWCNT. Future work investigating the role of TSP-1 at time points past $24 \mathrm{hrs}$ are necessary. The long-term consequences of pulmonary exposure to MWCNT are unclear, but there is increasing evidence support a link between MWCNT exposure and development of atherosclerosis. Apolipoprotein E -/- mice fed a western (high fat) diet in conjunction with chronic exposure to MWCNT have been observed exhibiting modest 
pulmonary inflammation (Han, 2016), oxidative damage to lung DNA, aortic remodeling (Christophersen et al., 2016), accelerated plaque progression (Cao et al., 2014), and enhanced expression of adhesion molecules (Suzuki et al., 2016). Human microvascular endothelial cells exposed to MWCNT either directly(Cao et al., 2014, Suzuki et al., 2016) or indirectly through co-culture(Snyder-Talkington et al., 2013) with lung epithelial cells respond with increased expression of adhesion molecules and oxidative stress. TSP-1 is a regulator of the cell adhesion molecules ICAM-1 and VCAM-1 (Narizhneva et al., 2005). These CAMs are involved in several important steps of atheroma formation (Obrien et al., 1993, Nakai et al., 1995, Nakashima et al., 1998, Choi et al., 2012) and elevated circulating TSP-1 is associated with development of atherosclerosis. (Choi et al., 2012). With these results in mind, it seems likely that TSP-1 may play a role in MWCNT-induced atherosclerosis which requires further investigation.

\section{Conclusions}

We have demonstrated for the first time that TSP-1 is a link between pulmonary nanomaterial exposure and peripheral changes in arteriolar function. In our study, lung MWCNT exposure in WT mice yielded increases in peripheral TSP-1 protein, enhanced leukocyte adhesion and rolling activity, and decreased arteriolar vasodilatory capacity.TSP-1 KO animals were largely protected from loss of vasodilatory response observed in arterioles of WT mice. This protection afforded to TSP-1 KO animals may be due to a combination of reduced leukocyte adhesion, enhanced NO signaling, reduced ROS stress, or some combination of all these mechanisms. Taken together, these data 
indicate a significant role for TSP-1 in mediating impaired peripheral microvascular reactivity following MWCNT exposure. More investigation is required to determine precisely what factors in the TSP-1 signaling pathway are involved, but likely it is the receptors CD47 and CD36. It will also be valuable to evaluate the role of TSP-1 over a period of days or weeks following MWCNT and other ENM exposure, especially in the context of atherosclerosis and other forms of CVD. 
Acknowledgements

This work was supported by the following sources: National Institutes of Health R01-ES015022 (TRN), the National Science Foundation Cooperative Agreement-1003907 (TRN), and DGE-1144676 (WKM). The authors would like to thank Carroll McBride and Michael Wolfarth for their expert technical assistance.

Declaration of interest

We have read and understood Nanotoxicology Journal policy on declaration of interests and declare that we have no competing interests. 


\section{References}

Aragon, M., Erdely, A., Bishop, L., Salmen, R., Weaver, J., Liu, J., Hall, P., Eye, T., Kodali, V., Zeidler-Erdely, P., Stafflinger, J.E., Ottens, A.K. \& Campen, M.J., 2016. Mmp9-Dependent Serum-Borne Bioactivity Caused By Multiwalled Carbon Nanotube Exposure Induces Vascular Dysfunction Via The Cd36 Scavenger Receptor. Toxicological Sciences, 150, 488-498.

Baenzige.NI, Brodie, G.N. \& Majerus, P.W., 1971. Thrombin-Sensitive Protein Of Human Platelet Membranes. Proceedings Of The National Academy Of Sciences Of The United States Of America, 68, 240-\&.

Bagher, P., Polo-Parada, L. \& Segal, S.S., 2011. Microiontophoresis And Micromanipulation For Intravital Fluorescence Imaging Of The Microcirculation. Journal Of Visualized Experiments : Jove.

Bauer, E.M., Qin, Y., Miller, T.W., Bandle, R.W., Csanyi, G., Pagano, P.J., Bauer, P.M., Schnermann, J., Roberts, D.D. \& Isenberg, J.S., 2010. Thrombospondin-1 Supports Blood Pressure By Limiting Enos Activation And Endothelial-Dependent Vasorelaxation. Cardiovascular Research, 88, 471-481.

Bearden, S.E. \& Segal, S.S., 2004. Motor Nerve Distribution Determines Feed Artery Control: Evidence From Mouse Gluteus Maximus Muscle. Faseb Journal, 18, Abst. 437.4-Abst. 437.4.

Bihari, P., Holzer, M., Praetner, M., Fent, J., Lerchenberger, M., Reichel, C.A., Rehberg, M., Lakatos, S. \& Krombach, F., 2010. Single-Walled Carbon Nanotubes Activate Platelets And Accelerate Thrombus Formation In The Microcirculation. Toxicology, 269, 148-154.

Bonner, J.C., Silva, R.M., Taylor, A.J., Brown, J.M., Hilderbrand, S.C., Castranova, V., Porter, D., Elder, A., Oberdorster, G., Harkema, J.R., Bramble, L.A., Kavanagh, T.J., Botta, D., Nel, A. \& Pinkerton, K.E., 2013. Interlaboratory Evaluation Of Rodent Pulmonary Responses To Engineered Nanomaterials: The Niehs Nano Go Consortium. Environmental Health Perspectives, 121, 676-682.

Borm, P.J.A. \& Mueller-Schulte, D., 2006. Nanoparticles In Drug Delivery And Environmental Exposure: Same Size, Same Risks? Nanomedicine, 1, 235-249.

Boulanger, C.M., 1999. Secondary Endothelial Dysfunction: Hypertension And Heart Failure. Journal Of Molecular And Cellular Cardiology, 31, 39-49.

Brown, E.J. \& Frazier, W.A., 2001. Integrin-Associated Protein (Cd47) And Its Ligands. Trends In Cell Biology, 11, 130-135.

Cai, H. \& Harrison, D.G., 2000. Endothelial Dysfunction In Cardiovascular Diseases - The Role Of Oxidant Stress. Circulation Research, 87, 840-844.

Cao, Y., Jacobsen, N.R., Danielsen, P.H., Lenz, A.G., Stoeger, T., Loft, S., Wallin, H., Roursgaard, M., Mikkelsen, L. \& Moller, P., 2014. Vascular Effects Of Multiwalled Carbon Nanotubes In Dyslipidemic Apoe(/) Mice And Cultured Endothelial Cells. Toxicological Sciences, 138, 104-116.

Celermajer, D.S., Sorensen, K.E., Gooch, V.M., Spiegelhalter, D.J., Miller, O.I., Sullivan, I.D., Lloyd, J.K. \& Deanfield, J.E., 1992. Noninvasive Detection Of Endothelial 
Dysfunction In Children And Adults At Risk Of Atherosclerosis. Lancet, 340, 11111115.

Chen, J.-T., Sun, H.-Q., Wang, W.-L., Xu, W.-M., He, Q., Shen, S., Qian, J. \& Gao, H.-L., 2015. Polyethylene Glycol Modification Decreases The Cardiac Toxicity Of Carbonaceous Dots In Mouse And Zebrafish Models. Acta Pharmacologica Sinica, $36,1349-1355$.

Cho, S., 2012. Cd36 As A Therapeutic Target For Endothelial Dysfunction In Stroke. Current Pharmaceutical Design, 18, 3721-3730.

Choi, K.Y., Kim, D.B., Kim, M.J., Kwon, B.J., Chang, S.Y., Jang, S.W., Cho, E.J., Rho, T.H. \& Kim, J.H., 2012. Higher Plasma Thrombospondin-1 Levels In Patients With Coronary Artery Disease And Diabetes Mellitus. Korean Circulation Journal, 42, 100-106.

Christophersen, D.V., Jacobsen, N.R., Andersen, M.H.G., Connell, S.P., Barfod, K.K., Thomsen, M.B., Miller, M.R., Duffin, R., Lykkesfeldt, J., Vogel, U., Wallin, H., Loft, S., Roursgaard, M. \& Moller, P., 2016. Cardiovascular Health Effects Of Oral And Pulmonary Exposure To Multi-Walled Carbon Nanotubes In Apoe-Deficient Mice. Toxicology, 371, 29-40.

Corbalan, J.J., Medina, C., Jacoby, A., Malinski, T. \& Radomski, M.W., 2011. Amorphous Silica Nanoparticles Trigger Nitric Oxide/Peroxynitrite Imbalance In Human Endothelial Cells: Inflammatory And Cytotoxic Effects. International Journal Of Nanomedicine, 6, 2821-2835.

Crouzier, D., Follot, S., Gentilhomme, E., Flahaut, E., Arnaud, R., Dabouis, V., Castellarin, C. \& Debouzy, J.C., 2010. Carbon Nanotubes Induce Inflammation But Decrease The Production Of Reactive Oxygen Species In Lung. Toxicology, 272, 39-45.

Fleming, I., Bauersachs, J. \& Busse, R., 1997. Calcium-Dependent And CalciumIndependent Activation Of The Endothelial No Synthase. Journal Of Vascular Research, 34, 165-174.

Flurkey, K., Currer, J. \& Harrison, D., 2007. The Mouse In Aging Research.

Gojova, A., Guo, B., Kota, R.S., Rutledge, J.C., Kennedy, I.M. \& Barakat, A.I., 2007. Induction Of Inflammation In Vascular Endothelial Cells By Metal Oxide Nanoparticles: Effect Of Particle Composition. Environmental Health Perspectives, 115, 403-409.

Han, S.G., 2016. Effects Of Multi-Walled Carbon Nanotubes In The Lungs And Aortas Of Apoe-Deficient Mice Fed A Normal Diet. Journal Of Nanoscience And Nanotechnology, 16, 8019-8024.

Inagami, T., Naruse, M. \& Hoover, R., 1995. Endothelium - As An Endocrine Organ. Annual Review Of Physiology, 57, 171-189.

Isenberg, J.S., Hyodo, F., Matsumoto, K.I., Romeo, M.J., Abu-Asab, M., Tsokos, M., Kuppusamy, P., Wink, D.A., Krishna, M.C. \& Roberts, D.D., 2007. Thrombospondin-1 Limits Ischemic Tissue Survival By Inhibiting Nitric OxideMediated Vascular Smooth Muscle Relaxation. Blood, 109, 1945-1952.

Isenberg, J.S., Ridnour, L.A., Dimitry, J., Frazier, W.A., Wink, D.A. \& Roberts, D.D., 2006a. Cd47 Is Necessary For Inhibition Of Nitric Oxide-Stimulated Vascular Cell 
Responses By Thrombospondin-1. Journal Of Biological Chemistry, 281, 2606926080.

Isenberg, J.S., Ridnour, L.A., Perruccio, E.M., Espey, M.G., Wink, D.A. \& Roberts, D.D., 2005a. Thrombospondin-1 Inhibits Endothelial Cell Responses To Nitric Oxide In A Cgmp-Dependent Manner. Proceedings Of The National Academy Of Sciences Of The United States Of America, 102, 13141-13146.

Isenberg, J.S., Ridnour, L.A., Perruccio, E.M., Espey, M.G., Wink, D.A. \& Roberts, D.D., 2005b. Thrombospondin-1 Inhibits Endothelial Cell Responses To Nitric Oxide In A Cgmp-Dependent Manner. Proceedings Of The National Academy Of Sciences Of The United States Of America, 102.

Isenberg, J.S., Wink, D.A. \& Roberts, D.D., 2006b. Thrombospondin-1 Antagonizes Nitric Oxide-Stimulated Vascular Smooth Muscle Cell Responses. Cardiovascular Research, 71, 785-793.

Jackson, D.N. \& Segal, S.S., 2006. Sex And Aging Interact To Modulate The Rapid Onset Of Arteriolar Dilation In Mouse Skeletal Muscle. Faseb Journal, 20, A271-A271.

Kong, P., Gonzalez-Quesada, C., Li, N., Cavalera, M., Lee, D.W. \& Frangogiannis, N.G., 2013. Thrombospondin-1 Regulates Adiposity And Metabolic Dysfunction In DietInduced Obesity Enhancing Adipose Inflammation And Stimulating Adipocyte Proliferation. American Journal Of Physiology-Endocrinology And Metabolism, 305, E439-E450.

Liu, X. \& Sun, J.A., 2010. Endothelial Cells Dysfunction Induced By Silica Nanoparticles Through Oxidative Stress Via Jnk/P53 And Nf-Kappa B Pathways. Biomaterials, 31, 8198-8209.

Louro, H., Tavares, A., Vital, N., Costa, P.M., Alverca, E., Zwart, E., De Jong, W.H., Fessard, V., Lavinha, J. \& Silva, M.J., 2014. Integrated Approach To The In Vivo Genotoxic Effects Of A Titanium Dioxide Nanomaterial Using Lacz Plasmid-Based Transgenic Mice. Environmental And Molecular Mutagenesis, 55, 500-509.

Matsuo, Y., Tanaka, M., Yamakage, H., Sasaki, Y., Muranaka, K., Hata, H., Ikai, I., Shimatsu, A., Inoue, M., Chun, T.H. \& Satoh-Asahara, N., 2015. Thrombospondin 1 As A Novel Biological Marker Of Obesity And Metabolic Syndrome. MetabolismClinical And Experimental, 64, 1490-1499.

Mercer, R.R., Hubbs, A.F., Scabilloni, J.F., Wang, L.Y., Battelli, L.A., Friend, S., Castranova, V. \& Porter, D.W., 2011. Pulmonary Fibrotic Response To Aspiration Of Multi-Walled Carbon Nanotubes. Particle And Fibre Toxicology, 8, 11.

Miller, T.W., Isenberg, J.S. \& Roberts, D.D., 2010. Thrombospondin-1 Is An Inhibitor Of Pharmacological Activation Of Soluble Guanylate Cyclase. British Journal Of Pharmacology, 159, 1542-1547.

Morimoto, Y., Horie, M., Kobayashi, N., Shinohara, N. \& Shimada, M., 2013. Inhalation Toxicity Assessment Of Carbon-Based Nanoparticles. Accounts Of Chemical Research, 46, 770-781.

Nakai, K., Itoh, C., Kawazoe, K., Miura, Y., Sotoyanagi, H., Hotta, K., Itoh, T., Kamata, J. \& Hiramori, K., 1995. Concentration Of Soluble Vascular Cell-Adhesion Molecule-1 (Vcam-1) Correlated With Expression Of Vcam-1 Messenger-Rna In The Human Atherosclerotic Aorta. Coronary Artery Disease, 6, 497-502. 
Nakashima, Y., Raines, E.W., Plump, A.S., Breslow, J.L. \& Ross, R., 1998. Upregulation Of Vcam-1 And Icam-1 At Atherosclerosis-Prone Sites On The Endothelium In The Apoe-Deficient Mouse. Arteriosclerosis Thrombosis And Vascular Biology, 18, 842851.

Narizhneva, N.V., Razorenova, O.V., Podrez, E.A., Chen, J.H., Chandrasekharan, U.M., Dicorleto, P.E., Plow, E.F., Topol, E.J. \& Byzova, T.V., 2005. Thrombospondin-1 Up-Regulates Expression Of Cell Adhesion Molecules And Promotes Monocyte Binding To Endothelium. Faseb Journal, 19, 1158-+.

Nemmar, A., Hoet, P.H.M., Vandervoort, P., Dinsdale, D., Nemery, B. \& Hoylaerts, M.F., 2007. Enhanced Peripheral Thrombogenicity After Lung Inflammation Is Mediated By Platelet-Leukocyte Activation: Role Of P-Selectin. Journal Of Thrombosis And Haemostasis, 5, 1217-1226.

Nurkiewicz, T.R., Porter, D.W., Barger, M., Castranova, V. \& Boegehold, M.A., 2004. Particulate Matter Exposure Impairs Systemic Microvascular EndotheliumDependent Dilation. Environmental Health Perspectives, 112, 1299-1306.

Nurkiewicz, T.R., Porter, D.W., Hubbs, A.F., Stone, S., Chen, B.T., Frazer, D.G., Boegehold, M.A. \& Castranova, V., 2009. Pulmonary Nanoparticle Exposure Disrupts Systemic Microvascular Nitric Oxide Signaling. Toxicological Sciences, 110, 191-203.

Obrien, K.D., Allen, M.D., Mcdonald, T.O., Chait, A., Harlan, J.M., Fishbein, D., Mccarty, J., Ferguson, M., Hudkins, K., Benjamin, C.D., Lobb, R. \& Alpers, C.E., 1993. Vascular Cell-Adhesion Molecule-1 Is Expressed In Human Coronary Atherosclerotic Plaques - Implications For The Mode Of Progression Of Advanced Coronary Atherosclerosis. Journal Of Clinical Investigation, 92, 945-951.

Panza, J.A., Quyyumi, A.A., Brush, J.E. \& Epstein, S.E., 1990. Abnormal EndotheliumDependent Vascular Relaxation In Patients With Essential-Hypertension. New England Journal Of Medicine, 323, 22-27.

Porter, D., Sriram, K., Wolfarth, M., Jefferson, A., Schwegler-Berry, D., Andrew, M. \& Castranova, V., 2008. A Biocompatible Medium For Nanoparticle Dispersion. Nanotoxicology, 2, 144-154.

Porter, D.W., Hubbs, A.F., Chen, B.T., Mckinney, W., Mercer, R.R., Wolfarth, M.G., Battelli, L., Wu, N., Sriram, K., Leonard, S., Andrew, M., Willard, P., Tsuruoka, S., Endo, M., Tsukada, T., Munekane, F., Frazer, D.G. \& Castranova, V., 2013. Acute Pulmonary Dose-Responses To Inhaled Multi-Walled Carbon Nanotubes. Nanotoxicology, 7, 1179-1194.

Porter, D.W., Hubbs, A.F., Mercer, R.R., Wu, N.Q., Wolfarth, M.G., Sriram, K., Leonard, S., Battelli, L., Schwegler-Berry, D., Friend, S., Andrew, M., Chen, B.T., Tsuruoka, S., Endo, M. \& Castranova, V., 2010. Mouse Pulmonary Dose- And Time CourseResponses Induced By Exposure To Multi-Walled Carbon Nanotubes. Toxicology, 269, 136-147.

Rao, G.V.S., Tinkle, S., Weissman, D.N., Antonini, J.M., Kashon, M.L., Salmen, R., Battelli, L.A., Willard, P.A., Hubbs, A.F. \& Hoover, M.D., 2003. Efficacy Of A Technique For Exposing The Mouse Lung To Particles Aspirated From The Pharynx. Journal Of Toxicology And Environmental Health-Part A, 66, 1441-1452. 
Roberts, D.D., Miller, T.W., Rogers, N.M., Yao, M.Y. \& Isenberg, J.S., 2012. The Matricellular Protein Thrombospondin-1 Globally Regulates Cardiovascular Function And Responses To Stress Via Cd47. Matrix Biology, 31, 162-169.

Schachinger, V., Britten, M.B. \& Zeiher, A.M., 2000. Prognostic Impact Of Coronary Vasodilator Dysfunction On Adverse Long-Term Outcome Of Coronary Heart Disease. Circulation, 101, 1899-1906.

Shaul, P.W., 2003. Endothelial Nitric Oxide Synthase, Caveolae And The Development Of Atherosclerosis. Journal Of Physiology-London, 547, 21-33.

Shvedova, A.A., Kisin, E., Murray, A.R., Johnson, V.J., Gorelik, O., Arepalli, S., Hubbs, A.F., Mercer, R.R., Keohavong, P., Sussman, N., Jin, J., Yin, J., Stone, S., Chen, B.T., Deye, G., Maynard, A., Castranova, V., Baron, P.A. \& Kagan, V.E., 2008. Inhalation Vs. Aspiration Of Single-Walled Carbon Nanotubes In C57bl/6 Mice: Inflammation, Fibrosis, Oxidative Stress, And Mutagenesis. American Journal Of Physiology-Lung Cellular And Molecular Physiology, 295, L552-L565.

Sinkler, S.Y. \& Segal, S.S., 2014. Aging Alters Reactivity Of Microvascular Resistance Networks In Mouse Gluteus Maximus Muscle. American Journal Of PhysiologyHeart And Circulatory Physiology, 307, H830-H839.

Smadja, D.M., D'audigier, C., Bieche, I., Evrard, S., Mauge, L., Dias, J.V., Labreuche, J., Laurendeau, I., Marsac, B., Dizier, B., Wagner-Ballon, O., Boisson-Vidal, C., Morandi, V., Duong-Van-Huyen, J.P., Bruneval, P., Dignat-George, F., Emmerich, J. \& Gaussem, P., 2011. Thrombospondin-1 Is A Plasmatic Marker Of Peripheral Arterial Disease That Modulates Endothelial Progenitor Cell Angiogenic Properties. Arteriosclerosis Thrombosis And Vascular Biology, 31, 551-559.

Snyder-Talkington, B.N., Schwegler-Berry, D., Castranova, V., Qian, Y. \& Guo, N.L., 2013. Multi-Walled Carbon Nanotubes Induce Human Microvascular Endothelial Cellular Effects In An Alveolar-Capillary Co-Culture With Small Airway Epithelial Cells. Particle And Fibre Toxicology, 10, 14.

Stapleton, P.A., Goodwill, A.G., James, M.E. \& Frisbee, J.C., 2007. Altered Mechanisms Of Endothelium-Dependent Dilation In Skeletal Muscle Arterioles With Genetic Hypercholesterolemia. American Journal Of Physiology-Regulatory Integrative And Comparative Physiology, 293, R1110-R1119.

Stapleton, P.A., Mcbride, C.R., Yi, J. \& Nurkiewicz, T.R., 2015. Uterine Microvascular Sensitivity To Nanomaterial Inhalation: An In Vivo Assessment. Toxicology And Applied Pharmacology, 288, 420-428.

Stapleton, P.A., Minarchick, V.C., Cumpston, A.M., Mckinney, W., Chen, B.T., Sager, T.M., Frazer, D.G., Mercer, R.R., Scabilloni, J., Andrew, M.E., Castranova, V. \& Nurkiewicz, T.R., 2012. Impairment Of Coronary Arteriolar EndotheliumDependent Dilation After Multi-Walled Carbon Nanotube Inhalation: A Time-Course Study. International Journal Of Molecular Sciences, 13, 13781-13803.

Steinberg, H.O., Chaker, H., Leaming, R., Johnson, A., Brechtel, G. \& Baron, A.D., 1996. Obesity/Insulin Resistance Is Associated With Endothelial Dysfunction Implications For The Syndrome Of Insulin Resistance. Journal Of Clinical Investigation, 97, 2601-2610. 
Stern, S.T., Adiseshaiah, P.P. \& Crist, R.M., 2012. Autophagy And Lysosomal Dysfunction As Emerging Mechanisms Of Nanomaterial Toxicity. Particle And Fibre Toxicology, 9.

Suzuki, Y., Tada-Oikawa, S., Hayashi, Y., Izuoka, K., Kataoka, M., Ichikawa, S., Wu, W.T., Zong, C., Ichihara, G. \& Ichihara, S., 2016. Single- And Double-Walled Carbon Nanotubes Enhance Atherosclerogenesis By Promoting Monocyte Adhesion To Endothelial Cells And Endothelial Progenitor Cell Dysfunction. Particle And Fibre Toxicology, 13, 11.

Thompson, L.C., Frasier, C.R., Sloan, R.C., Mann, E.E., Harrison, B.S., Brown, J.M., Brown, D.A. \& Wingard, C.J., 2014. Pulmonary Instillation Of Multi-Walled Carbon Nanotubes Promotes Coronary Vasoconstriction And Exacerbates Injury In Isolated Hearts. Nanotoxicology, 8, 38-49.

Thompson, L.C., Holland, N.A., Snyder, R.J., Luo, B., Becak, D.P., Odom, J.T., Harrison, B.S., Brown, J.M., Gowdy, K.M. \& Wingard, C.J., 2016. Pulmonary Instillation Of Mwcnt Increases Lung Permeability, Decreases Gp130 Expression In The Lungs, And Initiates Cardiovascular Il-6 Transsignaling. American Journal Of PhysiologyLung Cellular And Molecular Physiology, 310, L142-L154.

Varma, V., Yao-Borengasser, A., Bodles, A.M., Rasouli, N., Phanavanh, B., Nolen, G.T., Kern, E.M., Nagarajan, R., Spencer, H.J., Lee, M.J., Fried, S.K., Mcgehee, R.E., Peterson, C.A. \& Kern, P.A., 2008. Thrombospondin-1 Is An Adipokine Associated With Obesity, Adipose Inflammation, And Insulin Resistance. Diabetes, 57, 432439.

Wight, T.N., Raugi, G.J., Mumby, S.M. \& Bornstein, P., 1985. Light Microscopic Immunolocation Of Thrombospondin In Human-Tissues. Journal Of Histochemistry \& Cytochemistry, 33, 295-302.

Yamawaki, H. \& Iwai, N., 2006. Cytotoxicity Of Water-Soluble Fullerene In Vascular Endothelial Cells. American Journal Of Physiology-Cell Physiology, 290.

Zhang, L., Wang, X., Miao, Y., Chen, Z., Qiang, P., Cui, L., Jing, H. \& Guo, Y., 2016. Magnetic Ferroferric Oxide Nanoparticles Induce Vascular Endothelial Cell Dysfunction And Inflammation By Disturbing Autophagy. Journal Of Hazardous Materials, 304, 186-195.

Zhu, M.T., Wang, B., Wang, Y., Yuan, L., Wang, H.J., Wang, M., Ouyang, H., Chai, Z.F., Feng, W.Y. \& Zhao, Y.L., 2011. Endothelial Dysfunction And Inflammation Induced By Iron Oxide Nanoparticle Exposure: Risk Factors For Early Atherosclerosis. Toxicology Letters, 203, 162-171. 


\section{Tables}

Table 4.1. Animal Characteristics. No significant differences were observed between groups in age, weight, or sex.

\begin{tabular}{|c|l|l|l|l|}
\cline { 2 - 5 } \multicolumn{1}{c|}{} & \multicolumn{4}{l|}{ Animal Characteristics } \\
\cline { 2 - 6 } \multicolumn{1}{c|}{} & WT+SHAM & WT+MWCNT & $\begin{array}{l}\text { TSP-1 } \\
\text { KO+SHAM }\end{array}$ & $\begin{array}{l}\text { TSP-1 } \\
\text { KO+MWCNT }\end{array}$ \\
\hline $\begin{array}{c}\text { N total mice } \\
\text { (male:female) }\end{array}$ & 14 & 15 & 18 & 16 \\
$(7: 7)$ & $(7: 8)$ & $16: 10)$ & $(8: 8)$ \\
\hline Age (weeks) & $16.5 \pm 0.6$ & $16.4 \pm 0.5$ & $16.6 \pm 0.4$ & $15.8 \pm 0.5$ \\
\hline Weight (g) & $26.5 \pm 0.8$ & $25.1 \pm 0.8$ & $26.5 \pm 0.7$ & $26.5 \pm 0.7$ \\
\hline
\end{tabular}


Table 4.2. Baseline vessel characteristics. No significant differences were observed between groups of vessels used for microiontophoresis experiments in baseline or passive diameter, or resting tone.

\begin{tabular}{|l|l|l|l|l|}
\cline { 2 - 5 } \multicolumn{1}{c|}{} & \multicolumn{4}{l|}{ Vessel Characteristics } \\
\cline { 2 - 5 } \multicolumn{1}{c|}{} & WT+SHAM & WT+MWCNT & $\begin{array}{l}\text { TSP-1 } \\
\text { KO+SHAM }\end{array}$ & $\begin{array}{l}\text { TSP-1 } \\
\text { KO+MWCNT }\end{array}$ \\
\hline $\mathrm{n}$ (vessels) & 21 & 15 & 17 & 16 \\
\hline Baseline Diameter $(\mu \mathrm{M})$ & $34.4 \pm 2.4$ & $34.9 \pm 2.0$ & $33.4 \pm 2.2$ & $34.4 \pm 3.3$ \\
\hline Passive Diameter $(\mu \mathrm{M})$ & $52.0 \pm 2.6$ & $49.65 \pm 2.6$ & $51.2 \pm 3.5$ & $57.5 \pm 3.3$ \\
\hline Resting Tone $(\%)$ & $28.4 \pm 3.6$ & $28.7 \pm 3.4$ & $33.3 \pm 3.4$ & $34.8 \pm 3.6$ \\
\hline
\end{tabular}


Figures

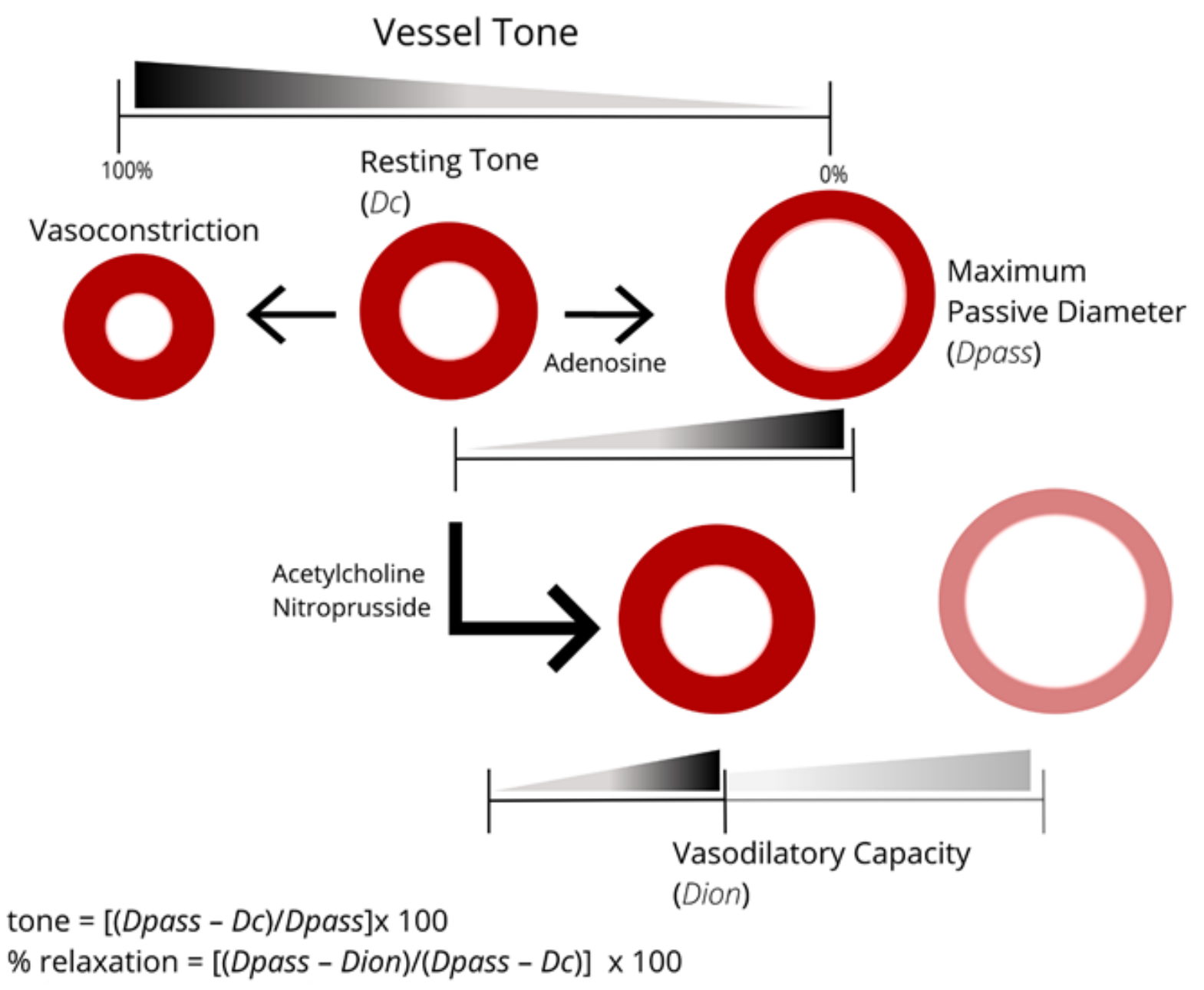

Figure 4.1 


\section{Skeletal Muscle TSP-1 Protein}
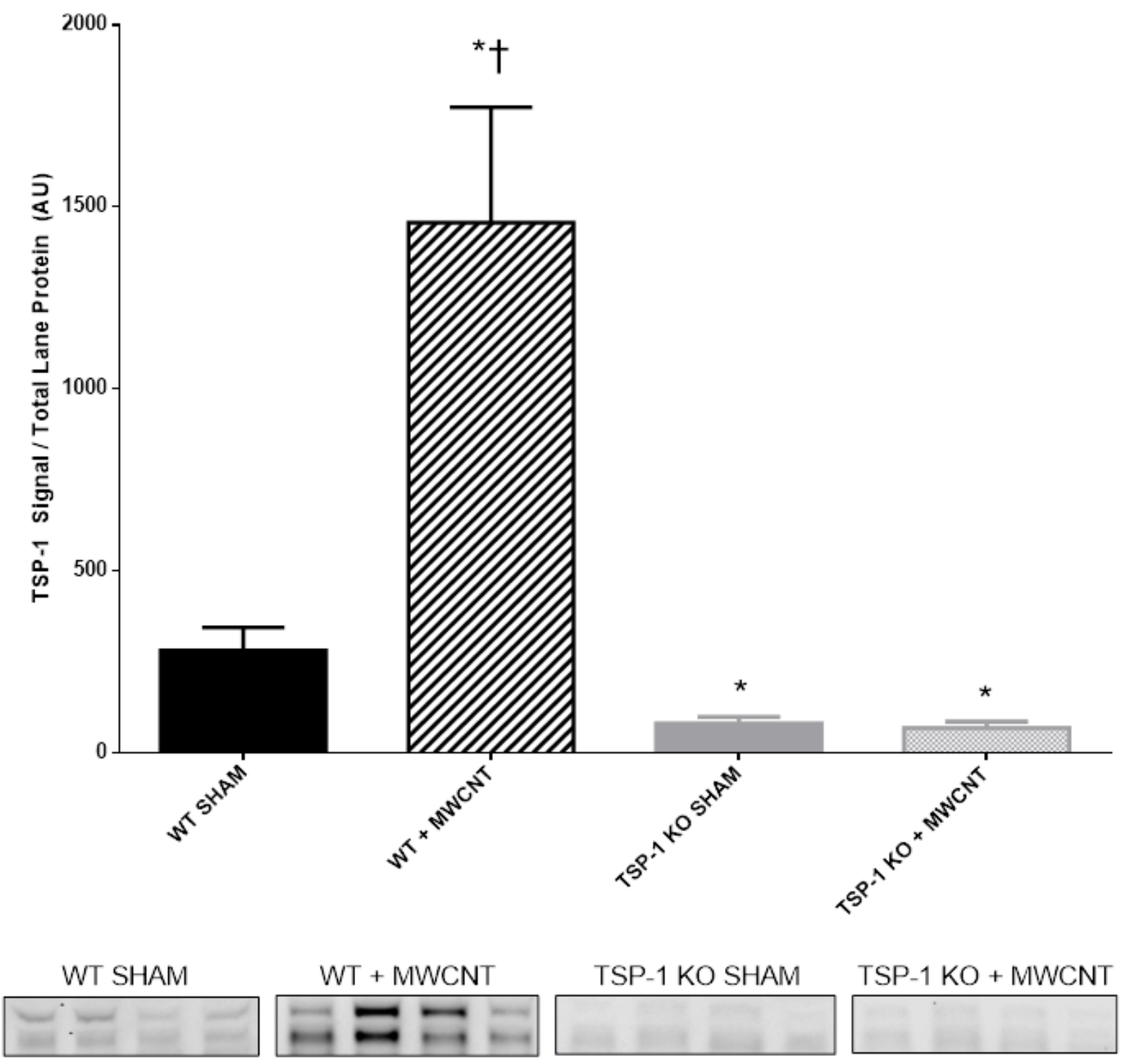

Figure 4.2 


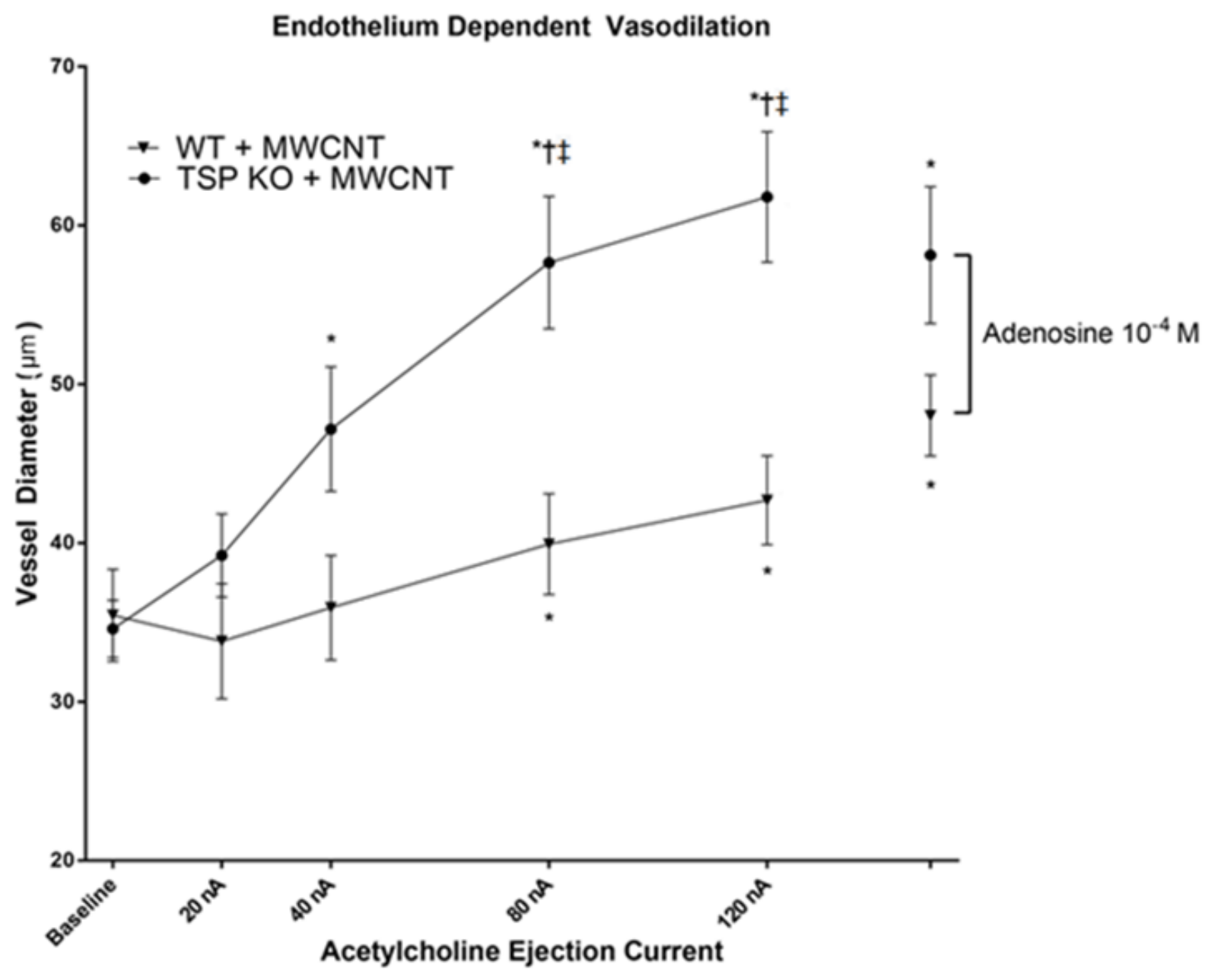

Figure 4.3 
A

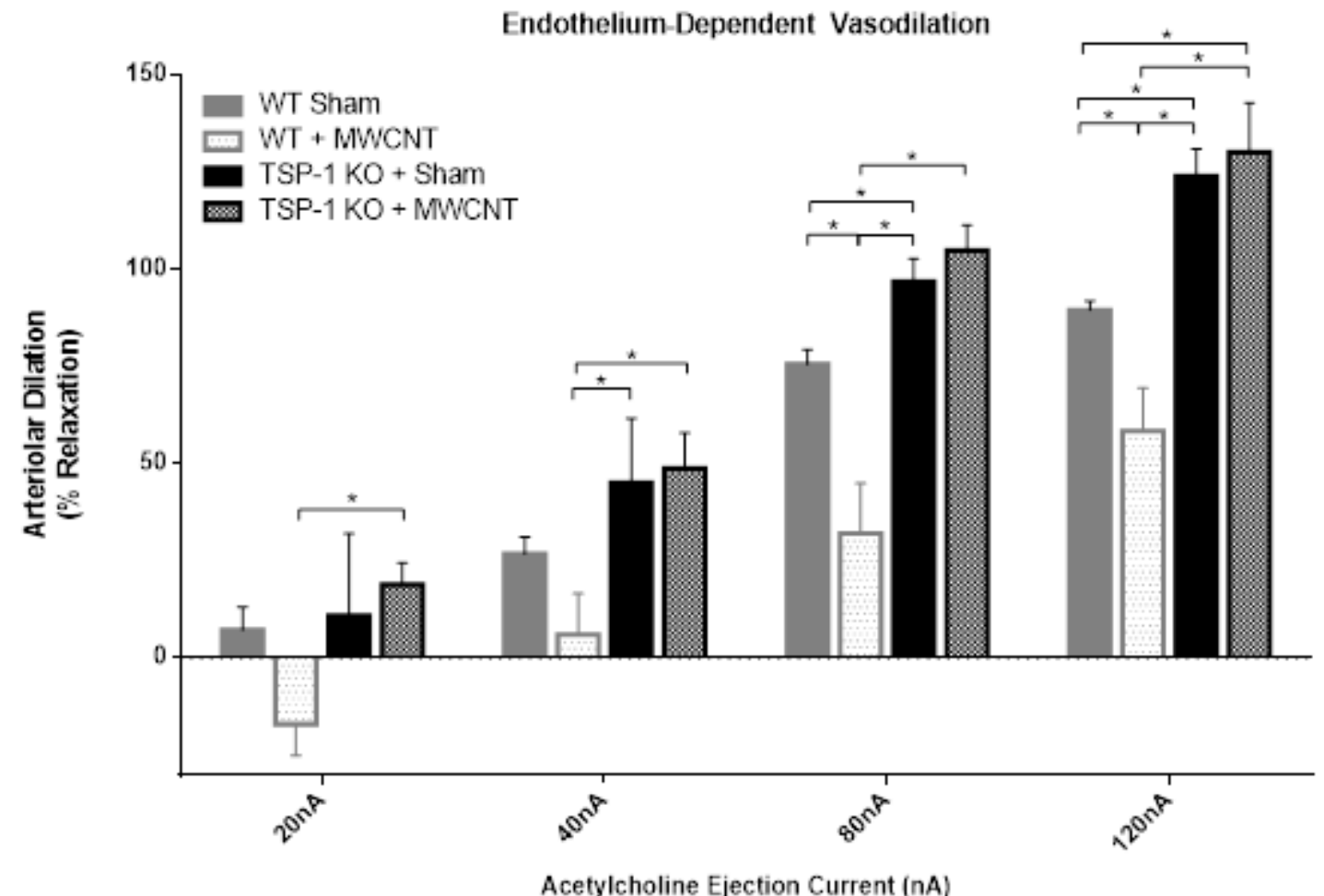

B

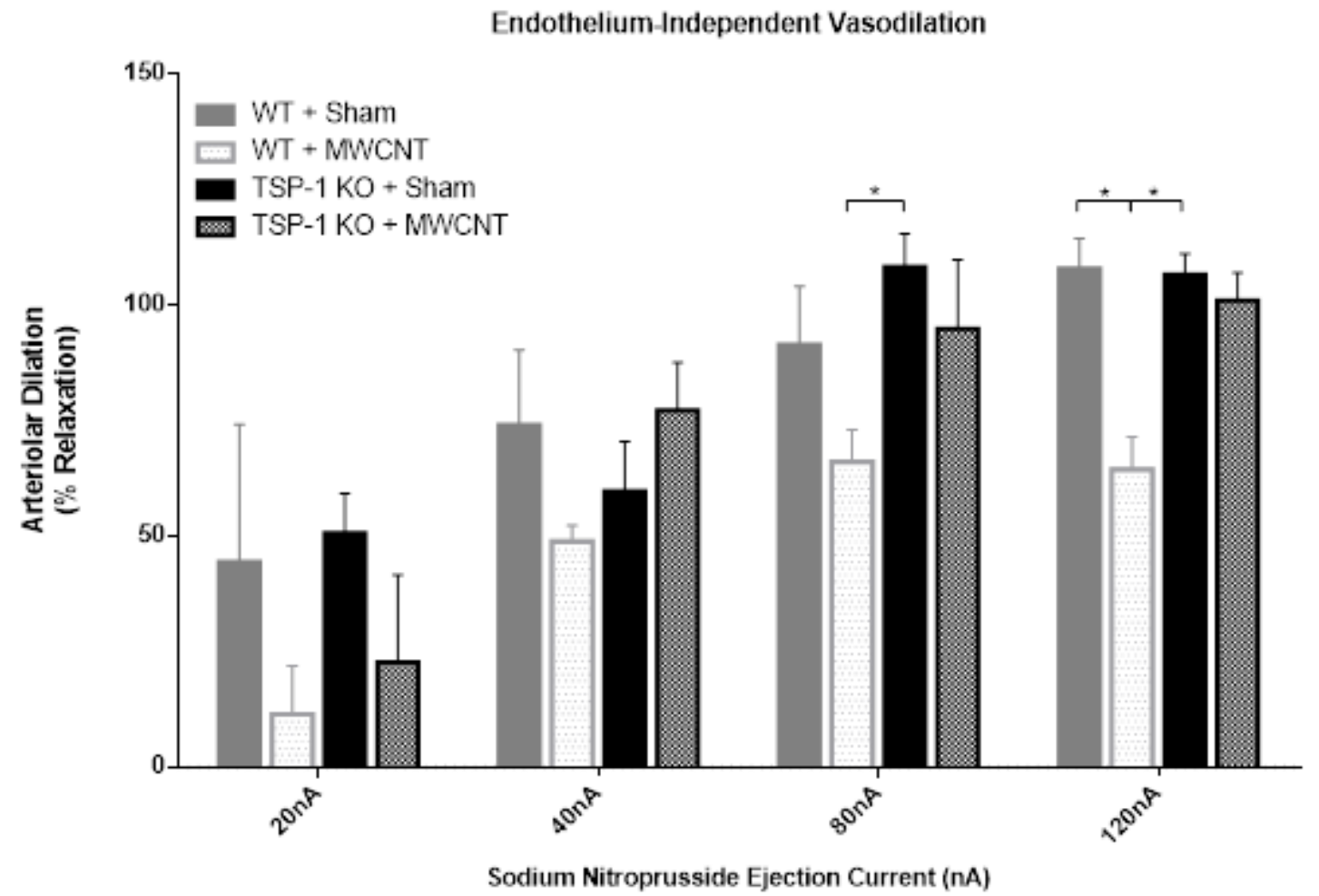

Figure 4.4 


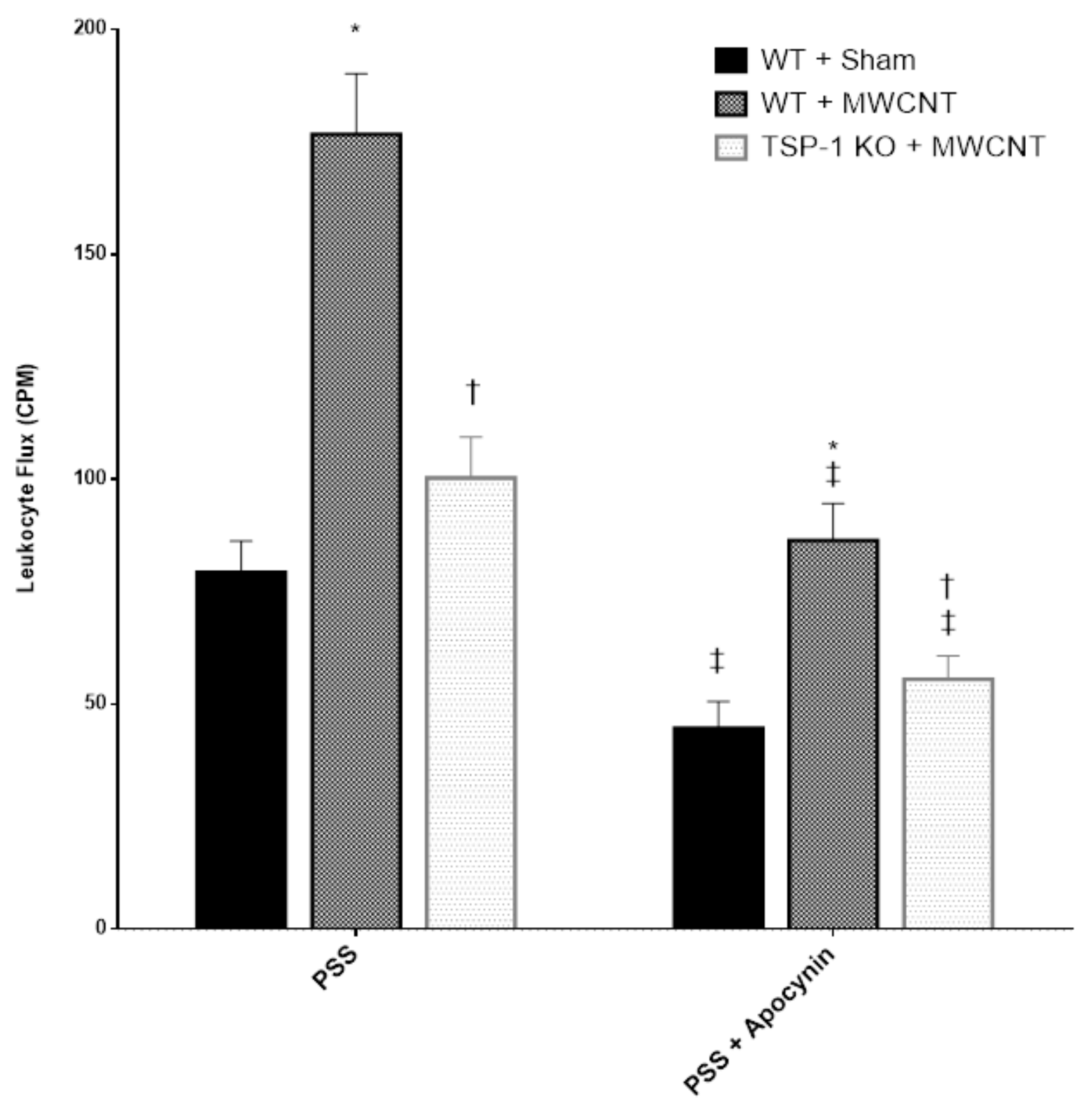

Figure 4.5 


\section{Figure Captions}

Figure 4.1 Graphical depiction of intravital microscopy experiments. Following an equilibration period, baseline vessel diameter is measured. Then, acetylcholine or sodium nitroprusside are applied via iontophoresis at varying currents to measure vessel reactivity. Finally adenosine is applied to measure passive diameter. Vessel reactivity is reported as a percentage of the passive diameter achieved with adenosine stimulation.

Figure 4.2 Skeletal muscle thrombospondin-1 protein level following lung multi-walled carbon nanotube exposure. TSP-1 protein increased by $512 \pm 21 \%$ in the WT+MWCNT compared to WT+SHAM group. TSP-1 KO protein levels were at the limit of detection. Values are mean $\pm \mathrm{SE}$. Lanes depict individual animals. $\mathrm{n}$ represents number of animals studied, $\left(^{*}\right)$ indicates difference from WT+SHAM. $\left({ }^{\dagger}\right)$ denotes difference from KO+MWCNT.

Figure 4.3 Arteriolar diameter following multi-walled carbon nanotube (MWCNT) aspiration. Representative vessel diameter following administration of acetylcholine (Ach) from wildtype (WT, $n=7$ ) and thrombospondin-1 (TSP-1, $n=12$ ) KO groups 24 hrs. following exposure to MWCNT. Vessel diameter curves for every group and drug condition are provided in supplemental figures 1 and $2 .\left(^{*}\right)$ indicates difference from baseline. $\left(^{\dagger}\right)$ indicates difference from previous ejection current value. (a) indicates difference between groups. Values are mean $\pm \mathrm{SE}$. $\mathrm{n}$ represents number of vessels studied 
Figure 4.4 Endothelium dependent and independent arteriole dilation following MWCNT aspiration. Acetylcholine $(A)$ dose response curves in WT + SHAM $(n=7)$, WT + MWCNT $(n=7)$, TSP-1 KO + SHAM (8), and TSP-1 KO + MWCNT $(n=12)$ and SNP dose response curves (B) WT + SHAM $(n=7), W T+M W C N T(n=7)$, TSP-1 KO + SHAM (5), and TSP-1 KO + MWCNT $(\mathrm{n}=8) 24$ hours post exposure. $\left(^{*}\right)$ indicates difference from $\mathrm{WT}+\mathrm{SHAM}$. Values are mean $\pm \mathrm{SE}$. $\mathrm{n}$ represents number of vessels studied.

Figure 4.5 Skeletal muscle venule leukocyte adhesion and rolling following lung multiwalled carbon nanotube aspiration. Leukocyte adhesion and rolling was enhanced in the WT+MWCNT group ( $n=13$ vessels) compared to WT+SHAM control group ( $n=13)$, but not the KO+MWCNT group $(n=14)$. Incubation with $10^{-4} \mathrm{M}$ apocynin reduced leukocyte flux in the WT+MWCNT to levels comparable to sham controls. $\left(^{*}\right)$ indicates difference from WT+SHAM. $\left({ }^{\dagger}\right)$ denotes difference from WT+MWCNT. ( $\left.\ddagger\right)$ indicates difference from PSS condition. Values are mean \pm SE. $n$ represents number of vessels studied. 


\section{Chapter 5: Microvascular dysfunction following multi walled carbon nanotube exposure is mediated by thrombospondin-1 receptor CD47}

W. Kyle Mandler ${ }^{1}$, Timothy R. Nurkiewicz ${ }^{2,3}$, Dale W. Porter ${ }^{4}$, Eric E. Kelley ${ }^{2,3}$

I. Mark Olfert ${ }^{1,3}$

${ }^{1}$ Division of Exercise Physiology, West Virginia University School of Medicine, Morgantown, WV 26506

${ }^{2}$ Department of Physiology and Pharmacology, West Virginia University School of Medicine, Morgantown, WV 26506

${ }^{3}$ Clinical and Translational Science Institute, West Virginia University, Robert C. Byrd Health Sciences Center, Morgantown, WV 26506

${ }^{4}$ National Institute for Occupational Safety and Health, Morgantown, WV 26505

Word count: 4,534

Short title: CD47 mediates microvascular endothelial dysfunction

\section{Corresponding Author:}

Mark Olfert, PhD, FAHA

West Virginia University School of Medicine

One Medical Center Dr.

PO BOX 9227

Morgantown, WV 26506

304-293-7597

imolfert@hsc.wvu.edu

Disclaimer: The findings and conclusions in this report are those of the authors and do not necessarily represent the views of the National Institute for Occupational Safety and Health. Mention of any company name or product does not constitute endorsement by NIOSH. 


\section{Abstract}

Pulmonary exposure to multi walled carbon nanotubes (MWCNT) and other nanomaterials have been shown to disrupt peripheral microvascular function. We recently demonstrated that levels of thrombospondin-1 (TSP-1) were increased in skeletal muscle following MWCNT exposure. TSP-1 is a matricellular glycoprotein highly expressed activated platelets during lung injury and inflammation. TSP-1 KO animals exhibited no impairments in skeletal muscle microvascular function following MWCNT exposure compared to WT controls. The mechanism through which TSP-1 exerts influence on vascular function in this state has yet to be determined, but we hypothesized that CD47 (a TSP-1 receptor) may be important for mediating changes in NO production, bioavailability, and signaling.

To test this, wildtype (WT) or CD47 knockout (CD47-KO) C57B6/J-background animals were exposed to $50 \mu \mathrm{g}$ of MWCNT or sham control via pharyngeal aspiration. 24 hrs. post-exposure intravital microscopy of the right gluteus maximus muscle was performed to assess arteriolar function via microiontophoretic delivery of varying doses (20, 40, 80, $120 \mathrm{nA0}$ of acetylcholine (ACh). Nearby venules were also imaged and recorded for 60 seconds to measure the number of adherent or rolling leukocytes. Immediately following the intravital procedure, lung, plasma, and left gluteus maximus tissues were collected for later analysis. Electron paramagnetic resonance (EPR) and NOx measurements were performed to assess tissue redox status, while inflammatory biomarkers were measured via multiplex assay. 
Endothelium-dependent vasodilation as stimulated by ACh was significantly impaired in the WT + MWCNT group compared to control (56.9 $\pm 8.9 \%$ vs $89.6 \pm 2.3 \%$ relaxation), while CD47 KO animals showed no impairment (108.46 $\pm 7.8 \%$ relaxation). Venular leukocyte adhesion and rolling activity was increased greater than $2 x$, while the CD47 KO group showed no change from WT control. Application of the antioxidant/NOX1 inhibitor apocynin rescued normal adhesion and rolling counts in the WT + MWCNT group. No differences were observed in blood $\mathrm{O}_{2}{ }^{\bullet-}$ levels as measured by EPR with the hydroxylamine spin probe, 1-hydroxy-4-phosphono-oxy-2,2,6,6-tetramethylpiperidine (PPH). Lung and plasma nitrate and nitrite $\left(\mathrm{NO}_{2}{ }^{-}\right.$and $\left.\mathrm{NO}_{3}{ }^{-}, \mathrm{NOx}\right)$ were reduced in the WT + MWCNT group by $47 \%$ and $32 \%$, respectively, while the CD47 KO group were unchanged from control. No differences were observed in either WT group with the biomarkers of inflammation we assessed, however, several pro-inflammatory cytokines were altered in the CD47 + MWCNT group; Lung IL-6, gluteus TNF-a, plasma IL-6, IL10, VEGF, and TNF-a were all upregulated, while lung VEGF was decreased.

These findings support the evidence that TSP-1 is an important ligand mediating MWCNT-induced microvascular dysfunction, and demonstrate that CD47 receptor is a key component of this dysregulation. In this model, CD47 activation likely disrupts NO signaling and promotes leukocyte-endothelial interactions. Impaired NO production, signaling, and bioavailability is etiologically linked to a variety of cardiovascular diseases. As such, the relevance of these results may not necessarily be limited only to vascular effects following pulmonary particular matter exposure, but possibly other pathologies where the TSP-1/CD47 axis is activated. 


\section{Introduction}

Engineered nanomaterials (ENM) are rapidly becoming an integral component of modern composite material manufacturing. Nanomaterials may be comprised of a wide range of chemical compositions and molecular structures, but by definition they must possess at least one dimension less than $100 \mathrm{~nm}$. This small scale provides advantages for manufacturing but nanomaterials are also more readily aerosolized and deposited into the deep lung upon inhalation, making them more dangerous than the same materials of larger particle size (Bakand et al., 2012). Of particular concern is a class of ENM known as multi-walled carbon nanotubes (MWNCT). While fairly heterogeneous in physicochemical properties (e.g. aspect ratio, metal contamination, attached function groups) all MWCNT are comprised of layers of graphene arranged in concentric tubes. Pulmonary symptoms of exposure to most MWCNT species include increased neutrophil influx, genotoxicity, lymphocytic aggregates, and fibrosis (Mercer et al., 2011)

There is evidence that in addition to direct damage to pulmonary tissue, exposure to nanomaterials is capable of generating deleterious systemic effects. The antivasodilatory effect for this route of exposure in arterioles has been previously established (LeBlanc et al., 2009, Nurkiewicz et al., 2009). As a primary source of peripheral resistance and control of tissue perfusion, the arterioles play a critical role in contributing to the maintenance of homeostasis and are responsive to a variety of chemical, neural, and physical inputs. The balance of these various inputs results in a physiological response of vasodilation or vasoconstriction achieved via changes in smooth muscle contraction or relaxation. Inability to control arteriolar diameter, and especially the ability 
to dilate appropriately, has been associated with a variety of serious disease states, including hypertension (Perticone et al., 2001, Petrak et al., 2006), peripheral/coronary artery disease (Heitzer et al., 2001, Brevetti et al., 2008), and atherosclerosis (Bonetti et al., 2003, Davignon and Ganz, 2004, Sitia et al., 2010). Pulmonary MWCNT exposure has been linked to each of the above conditions (Stapleton et al., 2012a, Cao et al., 2014, Thompson et al., 2014, Aragon et al., 2016, Han, 2016).

The molecular mechanisms linking pulmonary MWCNT exposure to microvascular dysfunction have yet to be fully elucidated. Among several putative pathways, one is that the innate immune response triggered in the lung activates and releases vasoactive factors which are then distributed systemically through the circulation. Acute lung injury and inflammation promotes macrophage influx, leukocyte adhesion (Inoue et al., 2009), and the formation of leukocyte-platelet aggregates (Zarbock and Ley, 2009). The matricellular matrix protein thrombospondin-1 (TSP-1) is a major component of the agranules of platelets, and is released upon platelet activation (Baenzige.NI et al., 1971). We have recently demonstrated that pulmonary exposure to MWCNT resulted in increased peripheral TSP-1 protein in wildtype C57B6] background mice, along with reduced arteriolar dilatory capacity, and increased leukocyte venular adhesion and rolling (Mandler et al., 2017). TSP-1 knockout mice exposed to MWCNT were protected from the negative changes in arteriolar reactivity experienced by wildtype control animals. TSP-1 elevation and impaired vascular function following MWCNT exposure have also recently been observed by another group (Aragon et al., 2016, Aragon et al., 2017). 
TSP-1 is capable of influencing vessel diameter via several distinct pathways. In particular, TSP-1 inhibits nitric oxide (NO) and cGMP signaling through the cell surface receptor CD47, which inhibits soluble guanylate cyclase (sGC) (Isenberg et al., 2005b). At picomolar concentrations, TSP-1 is capable of completely inhibiting NO-stimulated production of cGMP by SGC, thus limiting vascular smooth muscle relaxation (Isenberg et al., 2006b). CD47 signaling is also capable of causing eNOS uncoupling and generation of ROS (Bauer et al., 2012). In addition to directly inhibiting NO signaling and production, it is also possible that TSP-1 may indirectly influence arteriolar diameter by promoting the recruitment of leukocytes to the endothelium through activation of intracellular and vascular adhesion molecules (ICAM-1, VCAM-1) (Narizhneva et al., 2005). As these activated leukocytes adhere, roll, flatten, and undergo trans-endothelial migration, production of superoxide by NADPH-oxidase can quench NO as it is produced, reducing its bioavailability (Hopps et al., 2009). Superoxide is rapidly converted to $\mathrm{H}_{2} \mathrm{O}_{2}$ by superoxide dismutase, and may exert effects on vascular reactivity independent of NO (Bauer et al., 2012, Knuckles et al., 2013). Local oxidative and nitrositive stress are hallmarks of leukocyte endothelial interactions (Kar and Kavdia, 2012).

While the evidence for the role of TSP-1 as a key mediator in the pathophysiology of microvasculature dysfunction following nanomaterial exposure is strong, the relative contribution of various pathways have yet to be determined. Additionally, the upstream signals of TSP-1 in the lung, circulation, and skeletal muscle in this context are unclear. The objective of the current work is to determine the role of CD47 in following pulmonary 
MWCNT exposure, identify potential inflammatory regulators of TSP-1, and measure changes in the redox status lung, blood, and skeletal muscle.

\section{Methods}

\section{Animals}

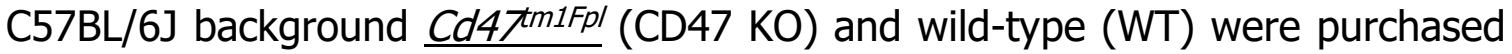
from Jackson Labs, Bar Harbor, ME (Stock\# 003173 and 000664, respectively). Mice were handled according to NIH Use of Laboratory Animals, Animal Welfare Act, and all experiments were approved by the West Virginia University Institutional Animal Care and Use Committee. Mice were kept on a 12-hour light/dark cycle, and provided food and tap water ad libitum. Experiments were scheduled so that data collection occurred at approximately 16 weeks of age. At this age, the mice are sexually and developmentally mature and the gluteus maximus muscle is of sufficient size and strength to undergo intravital microscopy (Flurkey et al., 2007). Both male and female WT and KO animals were used in all experiments in approximately equal numbers (Table 1).

\section{Nanomaterial exposure details}

MWCNT are catalytically grown by chemical vapor deposition processes and were obtained from Mitsui \& Co (MWNT-7, Lot \# 061220-31, Ibaraki, Japan). Mitsui-7 MWCNTs were designated as the ENM of choice for this project based on the wide number of potential applications, observed toxicity, and prior investigations by our group (Porter et al., 2010, Mandler et al., 2017). Oropharyngeal aspiration, as opposed to intratracheal instillation or aerosol inhalation was chosen as the method of exposure for this study due 
to the high degree of flexibility and scalability compared to inhalation, and is less traumatic for the animals than intratracheal instillation (Rao et al., 2003). Prior studies have shown that aspiration of this dose of MWCNT in dispersion medium (DM) generates pulmonary response analogous to instillation (Bonner et al., 2013) and inhalation (Shvedova et al., 2008, Mercer et al., 2011). In brief, prior to aspiration, $50 \mu \mathrm{g}$ MWCNT is suspended and dispersed in a phospho-buffered saline/purified mouse serum albumin (PBS/MSA) medium using ultrasonication. While anesthetized (isoflurane), the solution is placed in the oropharynx with the tongue manually extended. Upon release, the tongue retracts and the mouse reflexively inhales, aspirating the material and depositing in the lung (Rao et al., 2003). This dose and delivery method has previously been shown to induce lung injury and inflammation (Porter et al., 2010). Control animals received a sham aspiration of medium without nanomaterials. This DM has been shown to be both biocompatible, non-immunogenic, and effective at dispersing MWCNT (Porter et al., 2008, Porter et al., 2010).

\section{Intravital microscopy and iontophoresis of mouse gluteus maximus arterioles}

At 24-h post-aspiration, KO or WT mice were anesthetized using thiobutabarbital (Inactin, $100 \mathrm{mg} / \mathrm{kg}$, i.p.) and placed on a thermo-probe regulated heating pad to maintain a $37^{\circ} \mathrm{C}$ core temperature. The trachea was intubated to ensure a patent airway. The right gluteus maximus muscle was surgically exteriorized for microscopic observation, leaving its innervation and all feed vessels intact. This site of observation was chosen as skeletal muscle is the largest global reservoir of the microvasculature and the largest 
source of active peripheral resistance. After exteriorization, the muscle was secured over an optical pedestal with 5-0 suture wire at its in situ length. The pedestal was sealed with stopcock grease to create a superfusate bath. Throughout the surgery and following experimental period, the muscle was continuously superfused with an electrolyte solution (119 mM NaCl, $25 \mathrm{mM} \mathrm{NaHCO}$, $6 \mathrm{mM} \mathrm{KCl}$, and $3.6 \mathrm{mM} \mathrm{CaCl}_{2}$ ), warmed to $35{ }^{\circ} \mathrm{C}$, and equilibrated with $95 \% \quad \mathrm{~N}_{2}-5 \% \quad \mathrm{CO}_{2}(\mathrm{pH}=7.35-7.40)$. Superfusate flow rate was maintained at $4 \mathrm{ml} / \mathrm{min}$ to minimize equilibration with atmospheric oxygen.

The animals were transferred to an intravital microscope coupled to a digital video camera. Observations were made with a 20X water immersion objective. A micropipette with an opening of 1-2 $\mu \mathrm{m}$ was filled with a $10^{-2} \mathrm{M}$ acetylcholine (to measure endothelium dependent vasodilation) solutions. The tip of the pipette was guided under microscope to a point adjacent to the adventitia of the target arteriole. During this time a holding current of $-200 \mathrm{nA}$ or $200 \mathrm{nA}$, respectively, was applied to the bath to prevent ACh/SNP leakage from the pipette. Once in place and following a 20-minute equilibration period, ejection currents of $20 \mathrm{nA}, 40 \mathrm{nA}$ and $80 \mathrm{nA}$, and $120 \mathrm{nA}$ (for $\mathrm{ACh}$ ) were applied to the bath for 5 minutes each in a randomized order, with a 5-minute washout period between each step. Following the final ACh washout period, adenosine was added to the superfusate at a concentration of $10^{-3} \mathrm{M}$ to determine vessel maximal passive diameter. Images were displayed on a high-resolution computer monitor and digitally captured in the last minute of each step for later analysis using Image] (ImageJ 1.46v, National Institutes of Health). One to three first order arterioles were studied per mouse. Measurements of vessel diameter were made from recorded images. Vessel reactivity is reported as percent of 
maximal diameter achieved. Resting vascular tone was calculated for each vessel as follows:tone $=\left[\frac{\text { Dpass }-D c}{\text { Dpass }}\right] \times 100$, where $\mathrm{D}$ is arteriolar diameter, in micrometers, and $D_{\text {pass }}$ is passive diameter under $A D O$, and $D_{c}$ is the diameter measured during the control period. $D_{c}$ was taken following a 20-minute equilibration and only if no change in vessel diameter had been observed for at least 1 minute prior. Percent relaxation was calculated at each ACh or SNP ejection current as follows: $\%$ relaxation $=\left[\frac{\text { Dpass }- \text { Dion }}{\text { Dpass }-D c}\right] \times 100$, where $D_{\text {ion }}$ is the vessel diameter following 5 minutes of drug delivery via iontophoresis.

\section{Leukocyte adhesion and rolling}

Leukocytes that were either stationary or moving but in constant contact with the venular wall for at least $200 \mu \mathrm{m}$ were counted for $1 \mathrm{~min}$ in each venule studied. Following the completion of iontophoresis studies and after a 20 -minute washout period, $10^{-4} \mathrm{M}$ apocynin was added to the superfusate to examine contributions of reactive oxygen species. Following a 30 minute apocynin incubation period, the measurements were repeated.

\section{$\underline{\text { Tissue Redox Status }}$}

At the conclusion of intravital experiments, $100 \mu$ of whole blood was collected from each experimental animal and incubated with the spin probe 4-phosphonooxy-2,2,6,6tetramethylpiperidine- $\mathrm{N}$-hydroxyl (PPH) for 10 minutes at a final concentration of $0.25 \mathrm{mM}$ before being snap frozen for later analysis. Electron paramagnetic resonance was used to detect superoxide $\left(\mathrm{O}_{2}{ }^{\bullet-}\right)$ and in biologic samples, spectra were obtained EPR spectra 
were obtained using a Bruker EMX EPR spectrometer equipped with a Bruker ER4119 HS resonator. Samples were prepared in $1.5 \mathrm{ml}$ plastic centrifuge tubes and were transferred to a quartz flat cell (Wilmad Glass Co, Buena, NJ) before spectra were obtained. Superoxide levels were estimated from the magnitude of the downfield peak height of the 3-line nitrogen hyperfine as well as the area under the curve and were expressed in arbitrary units. Validation of superoxide was accomplished by the presence of SOD.

NO has an extremely short half-life in vivo and is rapidly converted to intermediates. Tissue $\mathrm{NO}_{x}$ (nitrate + nitrite) was measured by first chemically reducing $\mathrm{NO}_{\mathrm{x}}$ to $\mathrm{NO}$ gas $\left(0.05 \mathrm{M}\right.$ vanadium chloride $\left(\mathrm{VCl}_{3}\right)$ in $1 \mathrm{M} \mathrm{HCl}$ at $\left.92^{\circ} \mathrm{C}\right)$ as described by (Braman and Hendrix, 1989) and then detecting the light generated by reaction of NO gas with 03 using a Sievers 280i NO analyzer (NOA) (Boulder, CO). A $50 \mathrm{ml}$ glass impinger vial containing $30 \mathrm{ml} \mathrm{VCl}_{3}(0.4 \mathrm{M}$ in $1 \mathrm{M} \mathrm{HCl})$ at $951 \mathrm{C}$. Helium gas was bubbled through the impinger vial, a condenser, a water trap containing $10 \mathrm{ml} \mathrm{NaOH}$, and was delivered to the NOA. A standard curve was generated (Liquid Program software v 3.22 PNN, Sievers) and used to calculate NO released by each injected sample. $25 \mu \mathrm{l}$ samples were run in duplicate and chemiluminescence analyzed by the NOA.

\section{Tissue Inflammatory Markers}

Inflammatory cytokines (IL-6, IL1- $\beta$, IL-10, VEGF, TGF- $\beta$, and TNF- $\alpha$ ) were quantified by U-Plex Meso Scale Discovery ${ }^{\circledR}$ multi-cytokine array (Meso Scale Diagnostics, Gaithersburg, MD, USA), according to manufacture instructions, in the lung, plasma, and gluteus maximus. 


\section{Statistics and calculations}

A D'Agostino \& Pearson omnibus normality test was used to assess homogeneity of variance. In cases where non-normal datasets were detected, statistical comparisons were made with Kruskal-Wallis tests and post-hoc Wilcoxon rank-sum tests for between subject effects where appropriate. A $2 \times 2$ factorial ANOVA was performed to compare the main effects of exposure (sham vs MWCNT) and genotype (WT vs KO) for each variable. When significant main effects were observed a Sidak post-hoc test was used to determine where significant differences existed between the groups. In all cases, $p<$ 0.05 was used to establish statistical significance. All data are reported as mean $\pm \mathrm{SEM}$, unless otherwise noted.

\section{Results}

\section{Animal and baseline vessel characteristics}

No differences were observed between groups in age or weight (Table 1). Ratios of male to female animals used were equal or very close to equal. Arterioles examined in this study averaged approximately $34.5 \mu \mathrm{m}$ in diameter across all group (Table 2). No differences between treatment groups were observed in baseline diameter, passive diameter, or resting tone (Table 2). There were also no significant sex differences in vessel baseline diameter, maximal passive diameter, or reactivity before and after nanomaterial exposure. 


\section{Arteriolar endothelium dependent reactivity}

Arteriolar endothelium dependent reactivity was measured via intravital microscopy. ACh administration yielded a dose-dependent increase in WT + SHAM arterioles, reaching $89.6 \%$ of passive diameter at the highest ACh ejection current (Fig. 1). The WT + MWCNT arterioles demonstrated impaired vasodilation at 40,80 and 120 nA ACh, achieving only $56.8 \%$ of passive diameter, an impairment of $32.8 \%$ compared to controls (Fig 1.). Vessels in CD47 KO animals exposed to MWCNT displayed no impaired ACh response, and even exceeded the control group at the 80nA level achieving $105.3 \%$ of passive diameter (Fig. 1).

\section{Venular leukocyte adhesion and rolling}

The flux of leukocytes through target venules was assessed at baseline and following application of antioxidant/NOX1 inhibitor apocynin. MWCNT exposure increased leukocyte flux by $222 \%$ compared to WT control (Fig. 2). MWCNT exposure elicited no significant increase in leukocyte flux in the CD47KO group. Application of apocynin rescued normal leukocyte flux in the WT + MWCNT group and depressed values in the other groups (Fig. 2).

\section{$\underline{\text { Tissue Redox Status }}$}

No differences between any groups were observed in $\mathrm{O}_{2}{ }^{\bullet-}$ content in whole blood using EPR with the spin probe agent PPH (Fig. 3). Tissue NOx was assessed using a 
Sievers NO analyzer. In the Lung, NOx was reduced by $47 \%$ in WT + MWCNT animals, compared to control, while NOx levels in CD47KO + MWCNT animals were not different from controls (Fig. 4). A similar pattern was observed in the plasma, with a $32.3 \%$ NOx reduction in WT + MWCNT animals and no change in the CD47KO + MWCNT group.

\section{Tissue Inflammatory Markers}

No differences in any measure were observed between WT groups, however several markers were different from control compared to the CD47KO + MWCNT group. In the lung, IL-6 was elevated and VEGF was depressed in CD47 + MWCNT animals. In the plasma, IL-6, IL-10, VEGF, and TNF-a were all elevated in CD47 KO + MWCNT animals compared to control and WT + MWCNT groups. In gluteus muscle tissue, VEGF was depressed, while TNF-a was enhanced in CD47 + MWCNT animals. No differences between any groups were observed in measures of TGF- $\beta$ or IL1- $\beta$. 


\section{Discussion}

The principal finding of this study is that the CD47 receptor plays a critical role in the vascular dysfunction associated with lung exposure to MWCNT. We observed that CD47KO + MWCNT animals exhibited similar, or even greater, levels of arteriolar endothelium-dependent vasodilation in response to ACh stimulation than WT+SHAM controls. TSP-1 normally circulates at a low $(0.22 \mathrm{nM})$, but vasoactive concentration (Csanyi et al., 2012) acting as a limiting factor to NO signaling. Removal of this "brake" influence through ablation of this signaling pathway. These findings closely resemble patterns dilation achieved by TSP-1KO animals in our previous work (Mandler et al., 2017), as well as arterioles from CD47KO and TSP-1KO animals observed in other intravital microscopy experiments (Bauer et al., 2010). When exposed to MWCNT, CD47KO animals exhibited no decrease in dilatory capacity compared to WT animals, in which relaxation was impaired by $32.8 \%$. The protection offered by CD47 knockout strongly suggests that CD47 is integral in mediating impairments following pulmonary MWCNT exposure.

One mechanism through which MWCNT exposure ultimately exerts its effects on peripheral microvasculature presumably involves impairment of NO generation, signaling, and/or bioavailability. Although we did not detect any changes in $\mathrm{O}_{2}{ }^{\bullet-}$ levels using EPR (perhaps due to the abundance of heme, free iron and protein in blood), our NOA results suggest that NO is depressed in lung and plasma following MWCNT, and that CD47 KO animals are protected from this effect. The failure to observe a difference in the skeletal muscle may be explained by the much smaller portion of tissue in contact with the 
circulatory system in the skeletal muscle, compared to the plasma or lung. Our leukocyte adhesion analysis may also yield some additional information on vessel ROS status. CD47 signaling is capable of activating NADPH oxidase-1 (NOX1) in, both, the endothelium (Csanyi et al., 2012) and circulating leukocyte (Barclay, 2009). Generating ROS promotes leukocyte adhesion through P-selectin mobilization (Granger and Senchenkova, 2010b). CD47 signaling also promotes leukocyte adhesion through ICAM-1/VCAM-1 activation (Lagadec et al., 2003, Narizhneva et al., 2005), which in turn activates NOX1 within minutes (Deem and Cook-Mills, 2004), and promotes endothelial disorganization and permeability (DelMaschio et al., 1996). CD47 KO animals have reduced leukocyte recruitment into dermal air pouches, and reduced transmigration even when stimulated with TNF-a. (Azcutia et al., 2012). In this study, CD47KO animals did not exhibit increased leukocyte flux following MWCNT exposure (compared to WT animals), and application of apocynin (an antioxidant/NOX1 inhibitor) rescued normal leukocyte flux in the WT + MWCNT group, and reduced levels to below PSS alone in WT + SHAM and CD47KO + MWCNT groups. Pulmonary nanoparticle exposure generates oxidative and nitrositive stress in the microvascular wall (Nurkiewicz et al., 2009, LeBlanc et al., 2010), our findings suggest that TSP-1/CD47 signaling contribute to this effect and promote endothelial cell adhesion and transmigration, which can further exacerbate endothelial dysfunction.

Aragon et al. have recently investigated the contributions of another TSP-1 receptor, CD36, to MWCNT-exposure induced vascular dysfunction (Aragon et al., 2016, Aragon et al., 2017). The authors observed that CD36KO aortic rings did not exhibit 
significant reductions in vasodilation reactivity following incubation in serum collected from MWCNT-exposed mice, despite increase in circulating TSP-1 protein, in contrast with WT aortas which were dramatically impaired at 4 and $24 \mathrm{hrs}$. post-exposure. In WT aortas incubated in serum from matrix metalloproteinase-9 (MMP-9) KO animals exposed to MWCNT, no significant impairment was noted. The authors postulate an MMP-9/TSP$1 / C D 36$ signaling axis mediates vascular impairments. More investigation is necessary to reconcile the findings of Aragon et al. with our work with TSP-1 and CD47KO animals. It is possible that ablation of a single TSP-1 signaling axis is sufficient to offer protection. It is important to note, however, significant differences in methodology that may account for differences in signaling pathways activated. Aragon et al. 2016, utilized an isolated ex vivo wire myographic preparation of mouse aorta segments. While certainly a valid and useful tool for explorations of vascular function, this technique differs in several important ways from our work. In the present study, the use of an in vivo preparation allowed for the examination of vessels as they actively function, not removed from neural, mechanical, and humoral inputs and without pre-constriction. Secondly, the aorta differs fundamentally in form and function from the resistance vasculature, so comparisons made between the two are limited by these factors. To reconcile our findings with those of Aragon et al., it will be necessary to perform similar in vivo intravital investigations of the arterioles of CD36KO mice following MWCNT exposure. Indeed, future research is warranted to determine the relative contributions of CD47 vs. CD36, as well as which downstream targets are predominantly responsible for microvascular dysfunction. 
Acute single exposure to MWCNT has been associated with pulmonary inflammation at the $24 \mathrm{hr}$ timepoint as assesed by BAL (polymorphonucleocyte count, albumin, lactate dehyrdoginase conent) (Porter et al., 2010). Some studies have found that BAL fluid and macrophages have elevated inflammatory cytokines (including TNFa, IL-6, IL-1a, and IL-1ß), as well as other markers of inflammation, such as lactate dehydrogenase, mucin, and surfactant protein-D (SP-D) at day 1 post MWCNT exposure (Han et al., 2010, Dong et al., 2015). IL1- $\beta$ and IL-18 secretion has also been shown to be stimulated by exposure to raw or functionalized MWCNT in cultured macrophages (Hamilton et al., 2013). In this study, we saw no significant difference tissue or circulating cytokines between MWCNT and sham exposed WT groups. Other studies have also shown no changes in cytokine production following MWCNT exposure. Mitchell et al. observed no inflammation or changes in lung mRNA IL-10 or IL-6 at 24 hours following MWCNT exposure (Mitchell et al., 2007). Elgrabli et al. observed that no changes in BAL mRNA in

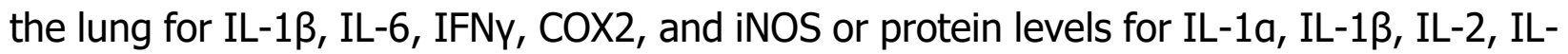
4, IL-6, IFNy, TNFa from 1 - 180 days post-MWCNT exposure (Elgrabli et al., 2008). At present, it is not clear why there are divergent responses, but most likely is due to differences in the methodology approaches and potentially the exposure paradigm.

We did, however, observe changes in several cytokines in our CD47 KO animals followng MWCNT exposure (Fig. 5). CD47 engagement can limit cytokine secretion (Demeure et al., 2000), while CD47 blocking in macrophages has the opposite effect (Weiskopf et al., 2015). It is possible that the cytokine changes observed are simply due to this effect, and not a result of MWCNT exposure. A limitation of our intepretation is 
the lack of an unexposed CD47KO group. Despite this, our results combined with those from other studies, allow us to make inferences about the role of the measured cytokines and their potential involvement in mediating peripheral endothelial dysfunction. The fact that in WT+MWCNT we observed no changes in cytokines in any tissues, but observed substantial inhibitions of vasodilation and increases in leukocyte adhesion, while in the CD47KO+MWCNT group the oppossite was true, strongly suggests that these cytokines, whether in the lung, circulation, or target organ are not playing a signicant role in mediating the observed microvascular dysfunction.

In summary, 24 hours following pulmonary exposure to MWCNT, CD47KO animals exhibited no loss of vasodilatory function and no change in leukocyte-endothelial interaction that is found in WT animals. No alterations in blood ROS status were observed, however MWCNT exposure lowered lung and plasma NOx in WT animals while CD47KO were unnaffected. Inflammatory cytokines were unchanged in WT animals following exposure, while a significant shift in pro-inflammatory factors was observed in CD47KO animals. These data complement earlier findings that microvascular dysfunction following MWCNT was TSP-1 dependent, and indicate that TSP-1/CD47 axis is an important mediator involved in the vascular dysfunction associated with exposure to MWCNTs. Our results are not necessarily limited to microvascular dysfunction following pulmonary exposure to nanomaterial. Indeed, TSP-1/CD47 signaling is activated in many disease states, including pulmonary arterial hypertension (Bauer et al., 2012), peripheral and coronary artery disease (Smadja et al., 2011, Choi et al., 2012), inclusion body myositis (Salajegheh et al., 2007) and others. Therefore, it is reasonable to speculate that ENM 
exposures which result in activation of this pathway may generate or exacerbate preexisting diseases. However, this must be directly tested in future studies. Therapuetic interventions aimed at the TSP-1/CD47 axis may help to reduce microvascular dysfunction not only in response to ENM exposure, but potentially other conditions that involve inhibition or reduced NO bioavailability. 


\section{Acknowledgements}

This work was supported by the following sources: National Institutes of Health R01-ES015022 (TRN), the National Science Foundation Cooperative Agreement-1003907 (TRN), and DGE-1144676 (WKM). The authors would like to thank Carroll McBride and Michael Wolfarth for their expert technical assistance. 


\section{References}

Aragon, M., Erdely, A., Bishop, L., Salmen, R., Weaver, J., Liu, J., Hall, P., Eye, T., Kodali, V., Zeidler-Erdely, P., Stafflinger, J.E., Ottens, A.K. \& Campen, M.J., 2016. Mmp-9-Dependent Serum-Borne Bioactivity Caused By Multiwalled Carbon Nanotube Exposure Induces Vascular Dysfunction Via The Cd36 Scavenger Receptor. Toxicological Sciences, 150, 488-498.

Aragon, M.J., Topper, L., Tyler, C.R., Sanchez, B., Zychowski, K., Young, T., Herbert, G., Hall, P., Erdely, A., Eye, T., Bishop, L., Saunders, S.A., Muldoon, P.P., Ottens, A.K. \& Campen, M.J., 2017. Serum-Borne Bioactivity Caused By Pulmonary Multiwalled Carbon Nanotubes Induces Neuroinflammation Via Blood-Brain Barrier Impairment. Proceedings Of The National Academy Of Sciences, 114, E1968.

Azcutia, V., Stefanidakis, M., Tsuboi, N., Mayadas, T., Croce, K.J., Fukuda, D., Aikawa, M., Newton, G. \& Luscinskas, F.W., 2012. Endothelial Cd47 Promotes Vascular Endothelial-Cadherin Tyrosine Phosphorylation And Participates In T Cell Recruitment At Sites Of Inflammation In Vivo. Journal Of Immunology, 189, 2553-2562.

Baenzige.NI, Brodie, G.N. \& Majerus, P.W., 1971. Thrombin-Sensitive Protein Of Human Platelet Membranes. Proceedings Of The National Academy Of Sciences Of The United States Of America, 68, 240-\&.

Bakand, S., Hayes, A. \& Dechsakulthorn, F., 2012. Nanoparticles: A Review Of Particle Toxicology Following Inhalation Exposure. Inhalation Toxicology, 24, 125-135.

Barclay, A.N., 2009. Signal Regulatory Protein Alpha (Sirpa) / Cd47 Interaction And Function. Current Opinion In Immunology, 21, 47-52.

Bauer, E.M., Qin, Y., Miller, T.W., Bandle, R.W., Csanyi, G., Pagano, P.J., Bauer, P.M., Schnermann, J., Roberts, D.D. \& Isenberg, J.S., 2010. Thrombospondin-1 Supports Blood Pressure By Limiting Enos Activation And Endothelial-Dependent Vasorelaxation. Cardiovascular Research, 88, 471-481.

Bauer, P.M., Bauer, E.M., Rogers, N.M., Yao, M.Y., Feijoo-Cuaresma, M., Pilewski, J.M., Champion, H.C., Zuckerbraun, B.S., Calzada, M.J. \& Isenberg, J.S., 2012. Activated Cd47 Promotes Pulmonary Arterial Hypertension Through Targeting Caveolin-1. Cardiovascular Research, 93, 682-693.

Bonetti, P.O., Lerman, L.O. \& Lerman, A., 2003. Endothelial Dysfunction - A Marker Of Atherosclerotic Risk. Arteriosclerosis Thrombosis And Vascular Biology, 23, 168175.

Bonner, J.C., Silva, R.M., Taylor, A.J., Brown, J.M., Hilderbrand, S.C., Castranova, V., Porter, D., Elder, A., Oberdorster, G., Harkema, J.R., Bramble, L.A., Kavanagh, T.J., Botta, D., Nel, A. \& Pinkerton, K.E., 2013. Interlaboratory Evaluation Of Rodent Pulmonary Responses To Engineered Nanomaterials: The Niehs Nano Go Consortium. Environmental Health Perspectives, 121, 676-682.

Braman, R.S. \& Hendrix, S.A., 1989. Nanogram Nitrite And Nitrate Determination In Environmental And Biological Materials By Vanadium(Iii) Reduction With Chemiluminescence Detection. Analytical Chemistry, 61, 2715-2718. 
Brevetti, G., Schiano, V. \& Chiariello, M., 2008. Endothelial Dysfunction: A Key To The Pathophysiology And Natural History Of Peripheral Arterial Disease? Atherosclerosis, 197, 1-11.

Cao, Y., Jacobsen, N.R., Danielsen, P.H., Lenz, A.G., Stoeger, T., Loft, S., Wallin, H., Roursgaard, M., Mikkelsen, L. \& Moller, P., 2014. Vascular Effects Of Multiwalled Carbon Nanotubes In Dyslipidemic Apoe(/) Mice And Cultured Endothelial Cells. Toxicological Sciences, 138, 104-116.

Choi, K.Y., Kim, D.B., Kim, M.J., Kwon, B.J., Chang, S.Y., Jang, S.W., Cho, E.J., Rho, T.H. \& Kim, J.H., 2012. Higher Plasma Thrombospondin-1 Levels In Patients With Coronary Artery Disease And Diabetes Mellitus. Korean Circulation Journal, 42, 100-106.

Csanyi, G., Yao, M., Rodriguez, A.I., Al Ghouleh, I., Sharifi-Sanjani, M., Frazziano, G., Huang, X., Kelley, E.E., Isenberg, J.S. \& Pagano, P.J., 2012. Thrombospondin-1 Regulates Blood Flow Via Cd47 Receptor Mediated Activation Of Nadph Oxidase 1. Arteriosclerosis Thrombosis And Vascular Biology, 32, 2966-+.

Davignon, J. \& Ganz, P., 2004. Role Of Endothelial Dysfunction In Atherosclerosis. Circulation, 109, 27-32.

Deem, T.L. \& Cook-Mills, J.M., 2004. Vascular Cell Adhesion Molecule 1 (Vcam-1) Activation Of Endothelial Cell Matrix Metalloproteinases: Role Of Reactive Oxygen Species. Blood, 104, 2385-2393.

Delmaschio, A., Zanetti, A., Corada, M., Rival, Y., Ruco, L., Lampugnani, M.G. \& Dejana, E., 1996. Polymorphonuclear Leukocyte Adhesion Triggers The Disorganization Of Endothelial Cell-To-Cell Adherens Junctions. Journal Of Cell Biology, 135, 497510.

Demeure, C.E., Tanaka, H., Mateo, V., Rubio, M., Delespesse, G. \& Sarfati, M., 2000. Cd47 Engagement Inhibits Cytokine Production And Maturation Of Human Dendritic Cells. Journal Of Immunology, 164, 2193-2199.

Dong, J., Porter, D.W., Batteli, L.A., Wolfarth, M.G., Richardson, D.L. \& Ma, Q., 2015. Pathologic And Molecular Profiling Of Rapid-Onset Fibrosis And Inflammation Induced By Multi-Walled Carbon Nanotubes. Archives Of Toxicology, 89, 621633.

Elgrabli, D., Abella-Gallart, S., Robidel, F., Rogerieux, F., Boczkowski, J. \& Lacroix, G., 2008. Induction Of Apoptosis And Absence Of Inflammation In Rat Lung After Intratracheal Instillation Of Multiwalled Carbon Nanotubes. Toxicology, 253, 131136.

Flurkey, K., Currer, J. \& Harrison, D., 2007. The Mouse In Aging Research.

Granger, D. \& Senchenkova, E., 2010. Chapter 7, Leukocyte-Endothelial Cell Adhesion. Inflammation And The Microcirculation. San Rafael, Ca: Morgan \&Amp; Claypool Life Sciences.

Hamilton, R.F., Xiang, C.C., Li, M., Ka, I., Yang, F., Ma, D.L., Porter, D.W., Wu, N.Q. \& Holian, A., 2013. Purification And Sidewall Functionalization Of Multiwalled Carbon Nanotubes And Resulting Bioactivity In Two Macrophage Models. Inhalation Toxicology, 25, 199-210. 
Han, S.G., 2016. Effects Of Multi-Walled Carbon Nanotubes In The Lungs And Aortas Of Apoe-Deficient Mice Fed A Normal Diet. Journal Of Nanoscience And Nanotechnology, 16, 8019-8024.

Han, S.G., Andrews, R. \& Gairola, C.G., 2010. Acute Pulmonary Response Of Mice To Multi-Wall Carbon Nanotubes. Inhalation Toxicology, 22, 340-347.

Heitzer, T., Schlinzig, T., Krohn, K., Meinertz, T. \& Munzel, T., 2001. Endothelial Dysfunction, Oxidative Stress, And Risk Of Cardiovascular Events In Patients With Coronary Artery Disease. Circulation, 104, 2673-2678.

Hopps, E., Lo Presti, R. \& Caimi, G., 2009. Pathophysiology Of Polymorphonuclear Leukocyte In Arterial Hypertension. Clinical Hemorheology And Microcirculation, 41, 209-218.

Inoue, K., Koike, E., Yanagisaw, R., Hirano, S., Nishikawa, M. \& Takano, H., 2009. Effects Of Multi-Walled Carbon Nanotubes On A Murine Allergic Airway Inflammation Model. Toxicology And Applied Pharmacology, 237, 306-316.

Isenberg, J.S., Ridnour, L.A., Perruccio, E.M., Espey, M.G., Wink, D.A. \& Roberts, D.D., 2005. Thrombospondin-1 Inhibits Endothelial Cell Responses To Nitric Oxide In A Cgmp-Dependent Manner. Proceedings Of The National Academy Of Sciences Of The United States Of America, 102.

Isenberg, J.S., Wink, D.A. \& Roberts, D.D., 2006. Thrombospondin-1 Antagonizes Nitric Oxide-Stimulated Vascular Smooth Muscle Cell Responses. Cardiovascular Research, 71, 785-793.

Kar, S. \& Kavdia, M., 2012. Local Oxidative And Nitrosative Stress Increases In The Microcirculation During Leukocytes-Endothelial Cell Interactions. Plos One, 7.

Knuckles, T.L., Stapleton, P.A., Minarchick, V.C., Esch, L., Mccawley, M., Hendryx, M. \& Nurkiewicz, T.R., 2013. Air Pollution Particulate Matter Collected From An Appalachian Mountaintop Mining Site Induces Microvascular Dysfunction. Microcirculation, 20, 158-169.

Lagadec, P., Dejoux, O., Ticchioni, M., Cottrez, F., Johansen, M., Brown, E.J. \& Bernard, A., 2003. Involvement Of A Cd47-Dependent Pathway In Platelet Adhesion On Inflamed Vascular Endothelium Under Flow. Blood, 101, 4836-4843.

Leblanc, A.J., Cumpston, J.L., Chen, B.T., Frazer, D., Castranova, V. \& Nurkiewicz, T.R., 2009. Nanoparticle Inhalation Impairs Endothelium-Dependent Vasodilation In Subepicardial Arterioles. Journal Of Toxicology And Environmental Health-Part ACurrent Issues, 72, 1576-1584.

Leblanc, A.J., Moseley, A.M., Chen, B.T., Frazer, D., Castranova, V. \& Nurkiewicz, T.R., 2010. Nanoparticle Inhalation Impairs Coronary Microvascular Reactivity Via A Local Reactive Oxygen Species-Dependent Mechanism. Cardiovascular Toxicology, 10, 27-36.

Mandler, W.K., Nurkiewicz, T.R., Porter, D.W. \& Olfert, I.M., 2017. Thrombospondin-1 Mediates Multi-Walled Carbon Nanotube Induced Impairment Of Arteriolar Dilation. Nanotoxicology, 11, 112-122.

Mercer, R.R., Hubbs, A.F., Scabilloni, J.F., Wang, L.Y., Battelli, L.A., Friend, S., Castranova, V. \& Porter, D.W., 2011. Pulmonary Fibrotic Response To Aspiration Of Multi-Walled Carbon Nanotubes. Particle And Fibre Toxicology, 8, 11. 
Mitchell, L.A., Gao, J., Wal, R.V., Gigliotti, A., Burchiel, S.W. \& Mcdonald, J.D., 2007. Pulmonary And Systemic Immune Response To Inhaled Multiwalled Carbon Nanotubes. Toxicological Sciences, 100, 203-214.

Narizhneva, N.V., Razorenova, O.V., Podrez, E.A., Chen, J.H., Chandrasekharan, U.M., Dicorleto, P.E., Plow, E.F., Topol, E.J. \& Byzova, T.V., 2005. Thrombospondin-1 Up-Regulates Expression Of Cell Adhesion Molecules And Promotes Monocyte Binding To Endothelium. Faseb Journal, 19, 1158-+.

Nurkiewicz, T.R., Porter, D.W., Hubbs, A.F., Stone, S., Chen, B.T., Frazer, D.G., Boegehold, M.A. \& Castranova, V., 2009. Pulmonary Nanoparticle Exposure Disrupts Systemic Microvascular Nitric Oxide Signaling. Toxicological Sciences, 110, 191-203.

Perticone, F., Ceravolo, R., Pujia, A., Ventura, G., Iacopino, S., Scozzafava, A., Ferraro, A., Chello, M., Mastroroberto, P., Verdecchia, P. \& Schillaci, G., 2001. Prognostic Significance Of Endothelial Dysfunction In Hypertensive Patients. Circulation, 104, 191-196.

Petrak, O., Widimsky, J., Jr., Zelinka, T., Kvasnicka, J., Strauch, B., Holaj, R., Stulc, T., Kvasnicka, T., Bilkova, J. \& Skrha, J., 2006. Biochemical Markers Of Endothelial Dysfunction In Patients With Endocrine And Essential Hypertension. Physiological Research, 55, 597-602.

Porter, D., Sriram, K., Wolfarth, M., Jefferson, A., Schwegler-Berry, D., Andrew, M. \& Castranova, V., 2008. A Biocompatible Medium For Nanoparticle Dispersion. Nanotoxicology, 2, 144-154.

Porter, D.W., Hubbs, A.F., Mercer, R.R., Wu, N.Q., Wolfarth, M.G., Sriram, K., Leonard, S., Battelli, L., Schwegler-Berry, D., Friend, S., Andrew, M., Chen, B.T., Tsuruoka, S., Endo, M. \& Castranova, V., 2010. Mouse Pulmonary Dose- And Time Course-Responses Induced By Exposure To Multi-Walled Carbon Nanotubes. Toxicology, 269, 136-147.

Rao, G.V.S., Tinkle, S., Weissman, D.N., Antonini, J.M., Kashon, M.L., Salmen, R., Battelli, L.A., Willard, P.A., Hubbs, A.F. \& Hoover, M.D., 2003. Efficacy Of A Technique For Exposing The Mouse Lung To Particles Aspirated From The Pharynx. Journal Of Toxicology And Environmental Health-Part A, 66, 1441-1452. Salajegheh, M., Raju, R., Schmidt, J. \& Dalakas, M.C., 2007. Upregulation Of Thrombospondin-1(Tsp-1) And Its Binding Partners, Cd36 And Cd47, In Sporadic Inclusion Body Myositis. Journal Of Neuroimmunology, 187, 166-174.

Shvedova, A.A., Kisin, E., Murray, A.R., Johnson, V.J., Gorelik, O., Arepalli, S., Hubbs, A.F., Mercer, R.R., Keohavong, P., Sussman, N., Jin, J., Yin, J., Stone, S., Chen, B.T., Deye, G., Maynard, A., Castranova, V., Baron, P.A. \& Kagan, V.E., 2008. Inhalation Vs. Aspiration Of Single-Walled Carbon Nanotubes In C57bl/6 Mice: Inflammation, Fibrosis, Oxidative Stress, And Mutagenesis. American Journal Of Physiology-Lung Cellular And Molecular Physiology, 295, L552-L565.

Sitia, S., Tomasoni, L., Atzeni, F., Ambrosio, G., Cordiano, C., Catapano, A., Tramontana, S., Perticone, F., Naccarato, P., Camici, P., Picano, E., Cortigiani, L., Bevilacqua, M., Milazzo, L., Cusi, D., Barlassina, C., Sarzi-Puttini, P. \& Turiel, M., 
2010. From Endothelial Dysfunction To Atherosclerosis. Autoimmunity Reviews, 9, 830-834.

Smadja, D.M., D'audigier, C., Bieche, I., Evrard, S., Mauge, L., Dias, J.V., Labreuche, J., Laurendeau, I., Marsac, B., Dizier, B., Wagner-Ballon, O., Boisson-Vidal, C., Morandi, V., Duong-Van-Huyen, J.P., Bruneval, P., Dignat-George, F., Emmerich, J. \& Gaussem, P., 2011. Thrombospondin-1 Is A Plasmatic Marker Of Peripheral Arterial Disease That Modulates Endothelial Progenitor Cell Angiogenic Properties. Arteriosclerosis Thrombosis And Vascular Biology, 31, 551-559.

Stapleton, P.A., Minarchick, V.C., Cumpston, A.M., Mckinney, W., Chen, B.T., Sager, T.M., Frazer, D.G., Mercer, R.R., Scabilloni, J., Andrew, M.E., Castranova, V. \& Nurkiewicz, T.R., 2012. Impairment Of Coronary Arteriolar EndotheliumDependent Dilation After Multi-Walled Carbon Nanotube Inhalation: A TimeCourse Study. International Journal Of Molecular Sciences, 13, 13781-13803.

Thompson, L.C., Frasier, C.R., Sloan, R.C., Mann, E.E., Harrison, B.S., Brown, J.M., Brown, D.A. \& Wingard, C.J., 2014. Pulmonary Instillation Of Multi-Walled Carbon Nanotubes Promotes Coronary Vasoconstriction And Exacerbates Injury In Isolated Hearts. Nanotoxicology, 8, 38-49.

Weiskopf, K., Ring, A., Garcia, K.C. \& Weissman, I., 2015. Cd47-Blocking Therapies Stimulate Macrophage Cytokine Secretion And Are Effective In A Model Of Peritoneal Carcinomatosis. Journal For Immunotherapy Of Cancer, 3, P248-P248. Zarbock, A. \& Ley, K., 2009. The Role Of Platelets In Acute Lung Injury (ALI). Frontiers In Bioscience, 14, 150-158. 


\section{Tables and Figures}

Table 5.1. Animal Characteristics. No significant differences were observed between groups in age, weight or sex.

\begin{tabular}{|l|l|l|l|}
\cline { 2 - 4 } \multicolumn{1}{c|}{} & \multicolumn{3}{l}{ Animal Characteristics } \\
\cline { 2 - 4 } \multicolumn{1}{c|}{} & WT+SHAM & WT+MWCNT & CD47 KO + MWCNT \\
\hline $\begin{array}{l}\text { N total mice } \\
\text { (male:female) }\end{array}$ & 14 & 15 & 6 \\
\hline Age (weeks) & $16: 7)$ & $(7: 8)$ & $(3: 3)$ \\
\hline Weight (g) & $26.5 \pm 0.6$ & $16.4 \pm 0.5$ & $16.1 \pm 0.2$ \\
\hline
\end{tabular}


Table 5.2 Baseline vessel characteristics. No significant differences were observed between exposure groups of vessels used for microiontophoresis experiments in baseline or passive diameter, or resting tone.

\begin{tabular}{|l|l|l|l|}
\cline { 2 - 4 } \multicolumn{1}{c|}{} & \multicolumn{3}{l|}{ Arteriole Characteristics } \\
\cline { 2 - 4 } \multicolumn{1}{c|}{} & WT+SHAM & WT+MWCNT & CD47KO \\
\hline $\mathrm{n}$ (vessels) & 21 & 15 & 8 \\
\hline Baseline Diameter $(\mu \mathrm{m})$ & $34.4 \pm 2.4$ & $34.9 \pm 2.0$ & $34.4 \pm 2.8$ \\
\hline Passive Diameter $(\mu \mathrm{m})$ & $52.0 \pm 2.6$ & $49.65 \pm 2.6$ & $47.5 \pm 3.5$ \\
\hline Resting Tone $(\%)$ & $32 \pm 2.8$ & $26 \pm 4.1$ & $27 \pm 3.2$ \\
\hline
\end{tabular}




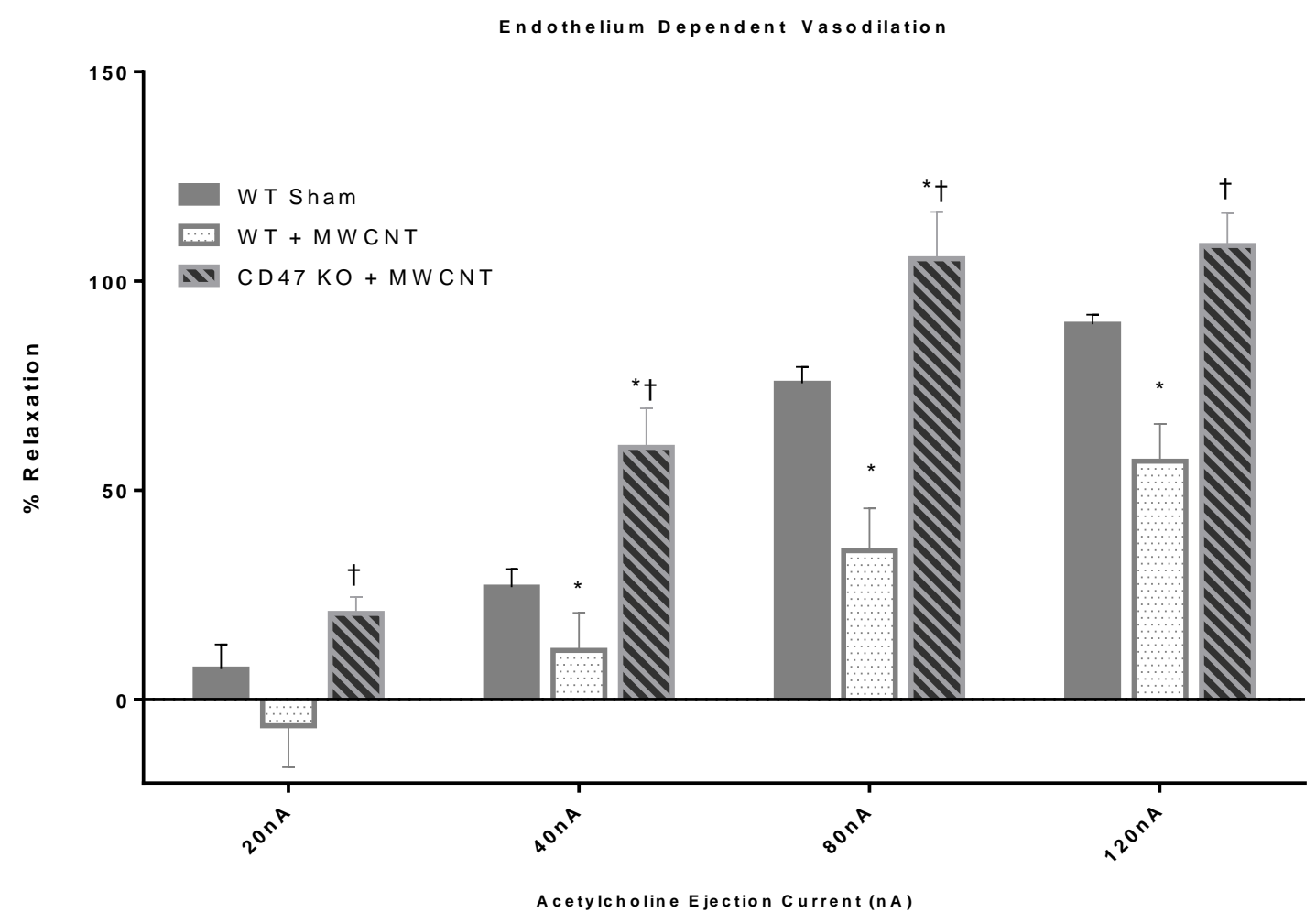

Figure 5.1. Endothelium dependent vasodilation in gluteus maximus arterioles.

Arteriolar dilation presented as percent of maximal passive dilation achieved with adenosine. $\left({ }^{*}\right)$ indicates difference $(p<0.05)$ from $W T+$ Sham group. $(\dagger)$ Indicates difference from WT + MWCNT group $(P<0.05)$. Number of earterioles studied for each group is as follows: WT + SHAM $(n=21)$, WT + $\operatorname{MWCNT}(n=15)$, CD47 KO + MWCNT $(n=8)$. 


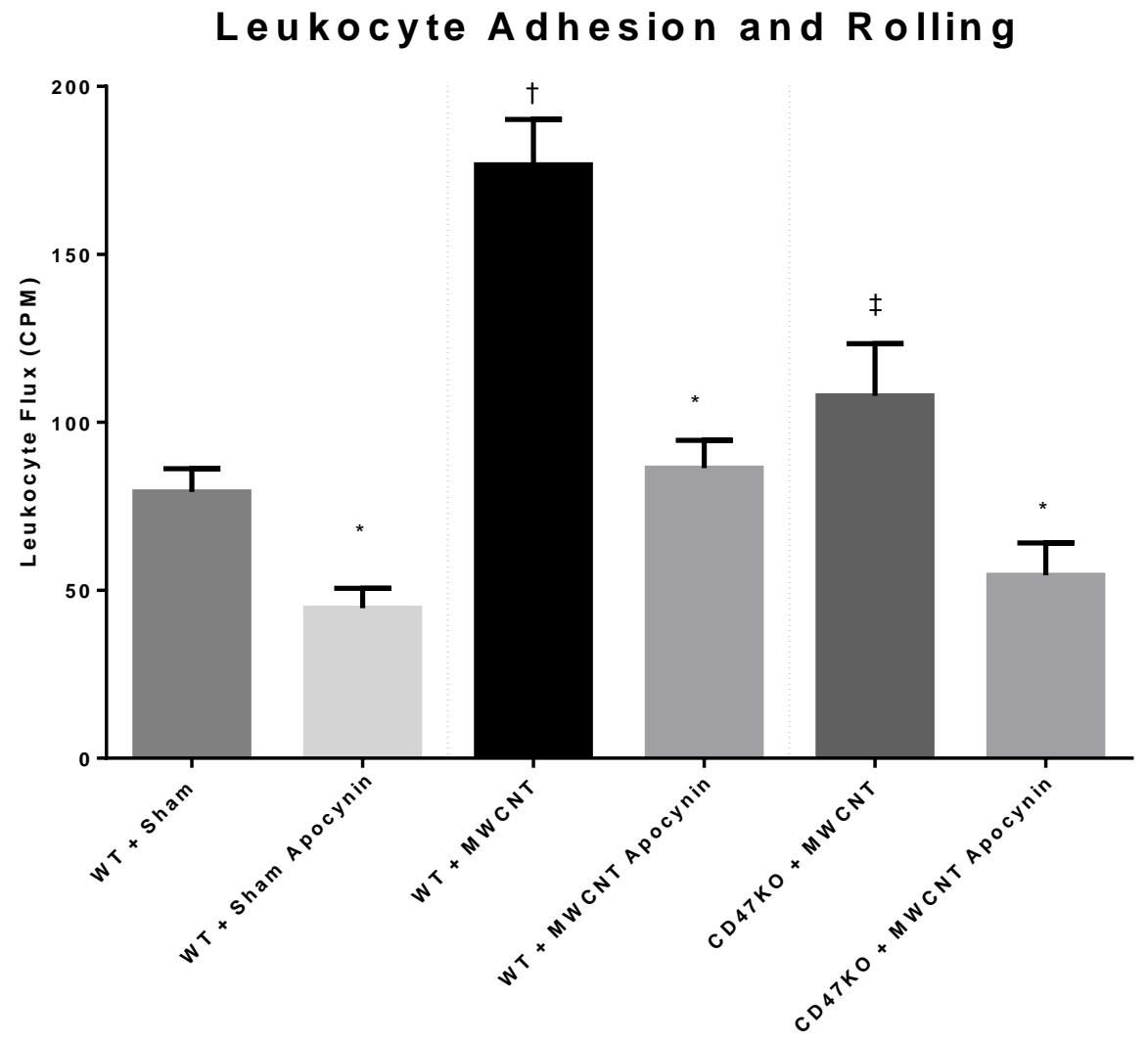

Figure 5.2. Venular leukocyte adhesion and rolling activity. $\left({ }^{*}\right)$ indicates main effect for Apocynin $(p<0.05)$. $(\dagger)$ indicates difference from WT + SHAM with PSS $(p<0.05)$. ( $\ddagger)$ Indicates difference from WT + MWCNT with PSS $(p<0.05)$. Number of venules studied for each group is as follows: WT + SHAM $(n=13)$, WT + MWCNT $(n=13)$, CD47 KO + MWCNT $(n=8)$. 


\section{Blood PPH spin probe EPR}
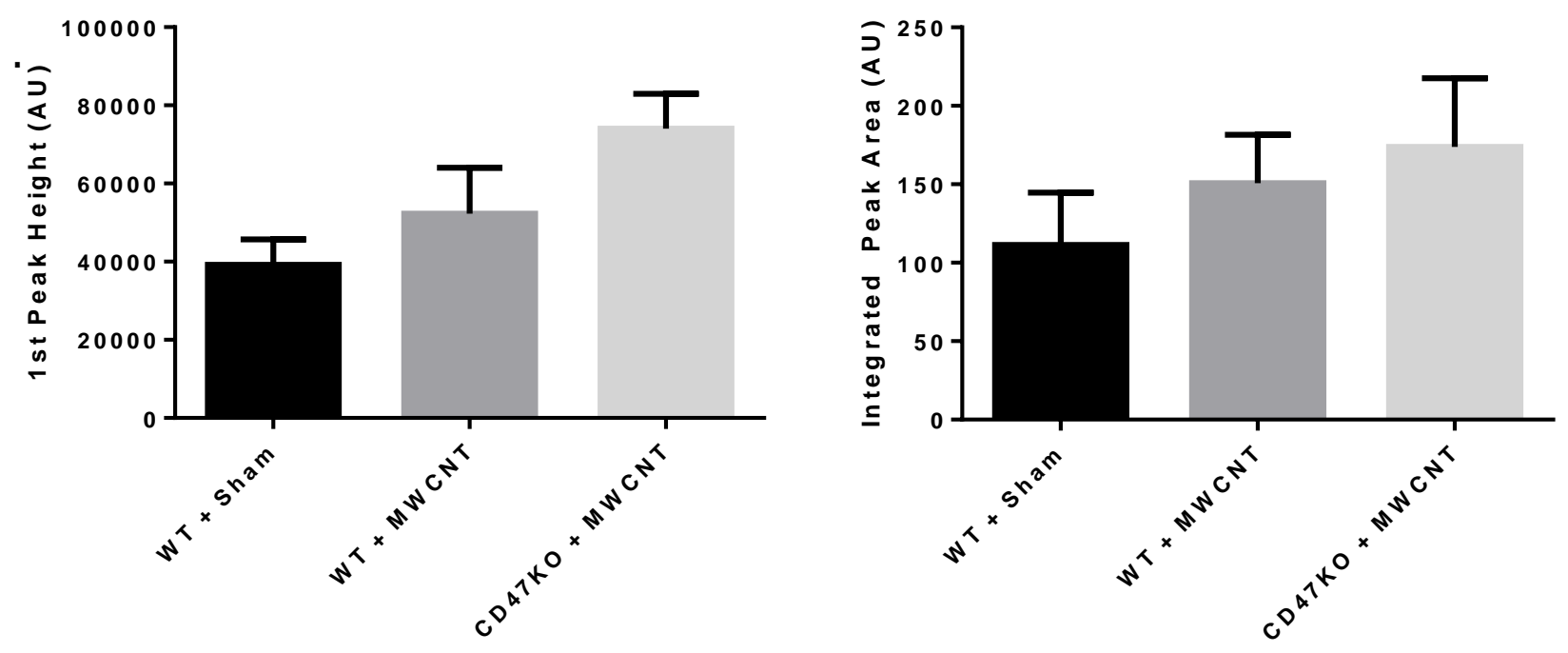

Figure 5.3 Blood reactive oxygen species content. No differences between groups were observed in either $1^{\text {st }}$ peak height $(A)$ or integrated peak area $(B)$ in blood samples using EPR with the spin trap compound PPH. Number of animals studied: WT + SHAM $(n=14)$, WT + MWCNT $(n=5)$, CD47 KO + MWCNT $(n=6)$. 
Tissue NOX
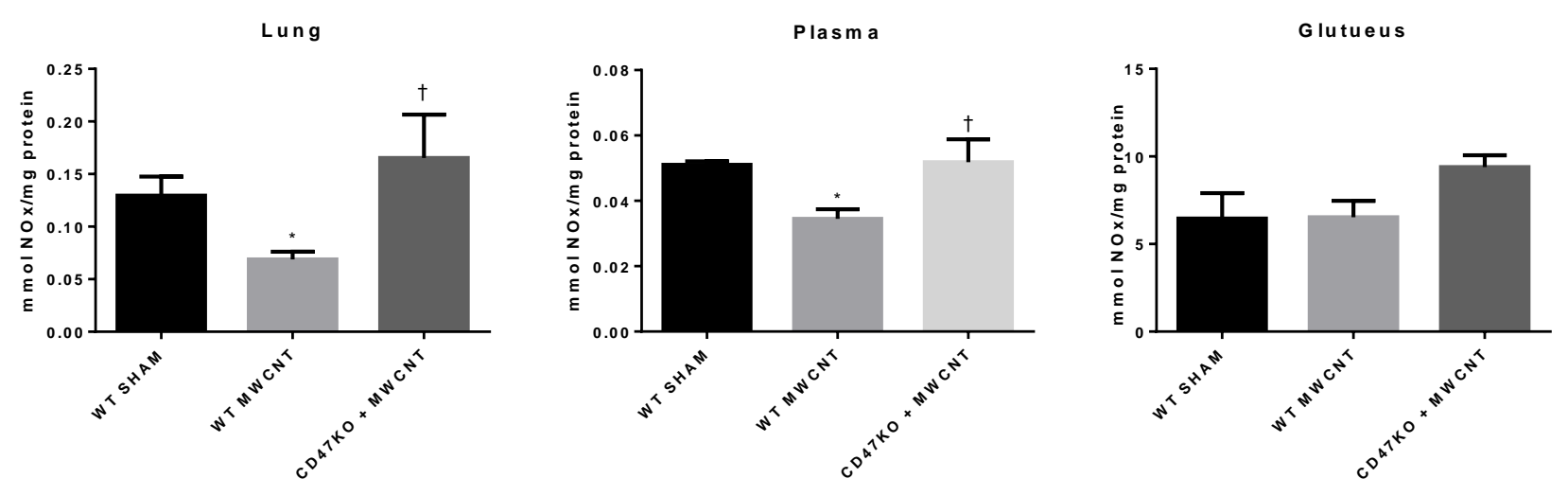

Figure 5.4 Tissue NOx. NOx was reduced in WT+MWCNT lung and plasma samples, compared to WT+SHAM $\left(^{*}\right)(p<0.05)$. NOx was elevated compared to WT+MWCNT in CD47KO+MWCNT lung and plasma $(\dagger)(p<0.05)$ No differences in between any groups were observed in the gluteus maximus tissues. Number of animals studied: WT + SHAM $(n=14)$, WT + MWCNT $(n=5)$, CD47 KO + MWCNT $(n=6)$ 
Tissue Inflammation Biomarkers
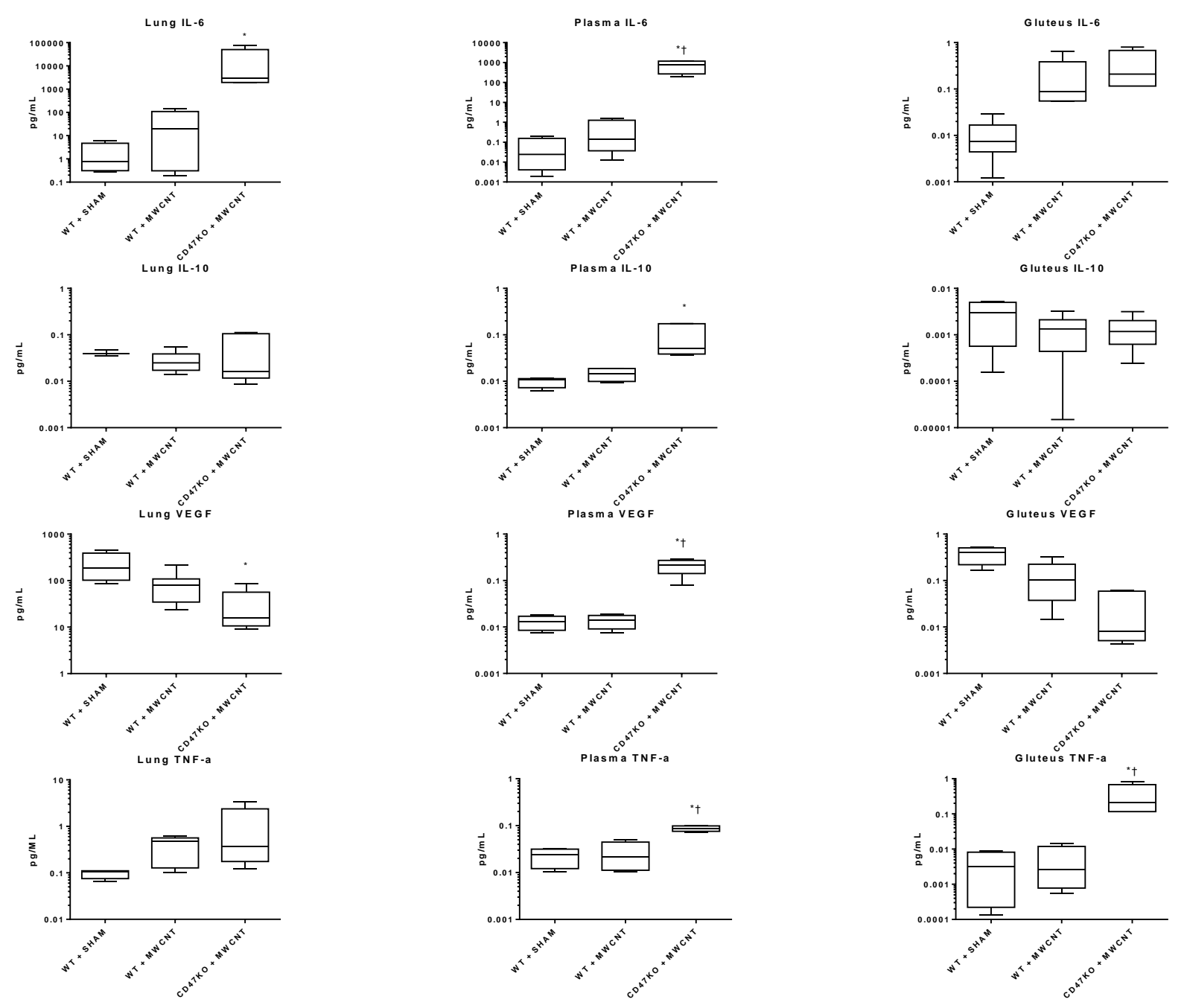

Figure 5.5 Tissue Inflammation Biomarkers. $\left(^{\star}\right)$ indicates difference from WT + SHAM group $(p<0.05)$. ( () Indicates difference from WT + MWCNT $(p<0.05)$. No differences were observed between groups in measured levels of or IL-1 Number of animals studied: WT + SHAM $(n=14)$, WT + MWCNT $(n=5)$, CD47 KO + MWCNT $(n=6)$. 
Chapter 6: General Discussion 
NO-mediated arteriolar reactivity plays a critical role in microvascular function and the maintenance of homeostasis. Dysregulation of this system is etiologically linked to a range of diverse pathologies including hypertension (Koller and Huang, 1994, Frisbee and Stepp, 2001) myocardial infarction (Michaels et al., 2000, Bolognese et al., 2004), Alzheimer's (Iadecola et al., 1999), and other vascular diseases (Hatoum et al., 2003). Pulmonary exposure to particulate matter and engineered nanomaterials has been shown to cause impairments of microvascular function. One mechanism responsible for these effects appears to be impairments of NO signaling and a shift towards oxidative stress (LeBlanc et al., 2009, Nurkiewicz et al., 2009, Stapleton et al., 2012). The work presented in Chapters 3 and 4 represent one of the first attempts to identify molecular pathways linking ENM-induced lung injury to peripheral vascular effects.

\section{AIM 1}

In order to properly investigate the molecular pathways activated by MWCNT exposure, it was necessary to first establish baseline arteriolar reactivity curves for the gluteus maximus arterioles using intravital microscopy in wildtype and KO animals. Previous investigations of the effects of particulate matter exposure on the microvasculature have typically used rats in tissues including the spinotrapezius (Nurkiewicz et al., 2004, Nurkiewicz et al., 2006, Nurkiewicz et al., 2008, Nurkiewicz et al., 2009, Knuckles et al., 2013), and uterine arterioles (Stapleton et al., 2015b). Compared to mice, rats have the practical advantages of greater tissue size and tolerance of invasive surgical procedures. However, availability of KO rats is limited and does not 
include the genes of interest for this study. In order to probe the function of TSP-1 and CD47 in the context of MWCNT exposure, it was necessary to use a mouse preparation.

Intravital microscopy of the mouse gluteus maximus arterioles was first developed by the Segal group and has since been utilized by them and others to study various aspects of microvascular function, especially changes with ageing (Bearden et al., 2004, Sinkler and Segal, 2014) both with iontophoresis (Bagher et al., 2011) and by adding vasoactive drugs to the entire superfusate solution (Givvimani et al., 2013). Iontophoresis offers several advantages over superfusate-wide dosing. Localized delivery allows reactivity to be studied at discrete locations and at specific orders within the microvascular network. This technique helps eliminate as confounding variables dramatic changes in blood flow, tissue perfusion, reflexive changes in blood pressure, thus exposing only the vasomotor properties of the target vessel. It is important to note that precise quantification of the amount of drug delivered is difficult to describe accurately, however the vessels consistently respond in an ejection current-dependent manner.

To date, there have only been a handful of studies utilizing intravital microscopy in transgenic or $\mathrm{KO}$ mice to study the microvasculature following lung particulate matter exposure (Sun et al., 2009, Xu et al., 2010, Kampfrath et al., 2011, Xu et al., 2012). These studies were conducted in the cremaster muscle, the animals were exposed to fine particulate matter $\left(\mathrm{PM}_{2.5}\right)$, where NOX2, TLR4, and p47(phos) were the genetic targets. The authors in these cases only investigated venular leukocyte adhesion and rolling activity. To our knowledge, the work presented in chapters 3 and 4 represent the first 
efforts to examine changes in arteriolar reactivity following pulmonary exposure to particulate matter in $\mathrm{KO}$ animals using this technique.

Our sham exposed TSP-1 and CD47 KO animals demonstrated enhanced vasodilation compared to WT controls reaching $130 \%$ and $112 \%$ of passive dilation achieved with adenosine (CD47 Sham unpublished data, $n=2$ ). The ability of the TSP-1 $\mathrm{KO}$ animals to achieve greater dilation is likely due to the anti-dilatory effects of TSP1/CD36 signaling still present in the CD47 KO mice. TSP-1 and CD47 KO arterial relaxation has been examined previously (Bauer et al., 2010). In that study, the authors observed 125 and $100 \%$ relaxation in TSP-1 and CD47 KO aortic rings, respectively. Comparisons between the observations of Bauer et al. 2010 in ex vivo wire myography preparations of mice aorta sections and our in vivo IVM work are of somewhat limited value due to the differences in preparation and drug delivery (iontophoresis vs. superfusate). In preliminary experiments not using iontophoresis, we observed that TSP-1 KO arterioles demonstrated only $98 \%$ of maximal passive diameter at a superfusate bath ACh concentration of $10^{-5} \mathrm{M}$ (Mandler et al., 2014). The differences between vessel dilation at similar $[\mathrm{ACh}]$ is likely caused by vasodilation of the entire vascular bed, leading to increased blood flow and shear stress, or reflexive sympathetic outflow present in our preparation that is absent in isolated vessel experiments.

One limitation of this work is the lack of blood pressure data for these experiments. The operational time frame available during this IVM procedure in mice is limited $(\sim 2$ hrs.), in order to maximize the amount of time available for measurement of arteriolar reactivity, blood pressure measurements were excluded. Future in vivo work should 
include pressure measurements as a matter of completeness, if possible. However, previous experiments have failed to show changes in mean arterial pressure after a single MWCNT exposure (Stapleton et al., 2012), so we do not believe that the lack of blood pressure would alter our interpretation of the data we obtained.

\section{AIM 2}

Having successfully demonstrated that our TSP-1 and CD47 KO gluteus maximus experimental prep correlates well with other ex vivo investigations of TSP-1 and CD47 KO vessel reactivity and established a functional baseline, we could now investigate vessel responses following MWCNT exposure. Our first objective was to determine the role of TSP-1. Elevated TSP-1 protein levels are associated with a variety of diseases in which deficits in NO production, bioavailability, and signaling play important roles, including PAH (Morrell et al., 2009, Labrousse-Arias et al., 2016), PAD (Brevetti et al., 2008, Smadja et al., 2011, Meller et al., 2013), CAD (Choi et al., 2012, Meller et al., 2013), diabetes (Stenina et al., 2003, Varma et al., 2008, Maier et al., 2010, Matsuo et al., 2015), and others. In preliminary experiments we observed increases in skeletal muscle TSP-1 levels in mice $24 \mathrm{hrs}$. following 2.5, 10, and $40 \mu \mathrm{g}$ MWCNT aspiration exposures. We also observed TSP-1 protein increases in rat skeletal muscle 24hrs post MWCNT inhalation exposures, but not after Cerium Oxide nanoparticle inhalation (Mandler et al., 2014). In Chapter 3, we observed a greater than fivefold increase in TSP-1 protein in WT mouse skeletal muscle after MWCNT aspiration (Mandler et al., 2017). Our observations were recently confirmed by Aragon et al. (Aragon et al., 2017). The authors observed significant increases in serum TSP-1 protein as well as dramatic increases in mRNA 
expression of several pro-inflammatory genes. TSP-1 elevation was associated with increased blood brain barrier permeability and neuroinflammation, which was ablated in TSP-1 receptor CD36 knockout animals. Taken in the context of earlier work linking CD36 KO with protection from MWCNT induced vascular reactivity, it is highly suggestive that TSP-1 is a mediator of this process. While TSP-1 has yet to be investigated in the context of other particulate matter exposure, it seems likely that in light of these findings that TSP-1 may be implicated in many instances where lung injury and inflammation occur.

We observed significant impairment in both endothelium-dependent (via ACh) and independent vasodilation (via SNP) vasodilation 24 hrs. post-MWCNT exposure in WT animals, suggesting impairments in both endothelium-dependent NO signaling as well as smooth muscle NO sensitivity. Alterations in reactivity to ACh or the calcium ionophore A23187 have been observed previously following MWCNT exposure, however our observation of reduced NO sensitivity are in contrast to several studies (Nurkiewicz et al., 2009, Stapleton et al., 2012). Differences observed between our SNP results and these are likely due to differences in experimental species or vascular bed, although more investigation into this question is warranted. In our TSP-1 KO animals, no impairment of ACh or SNP mediated vasodilation was observed at any ejection current level. These findings represent the first implication of TSP-1 in mediating alterations in arteriolar dilation following MWCNT exposure.

\section{AIM 3}

Identifying TSP-1 as a mediator of MWCNT-induced microvascular dysfunction is an important step in understanding of this phenomena. However, to eventually be able 
to treat or prevent it, it is necessary to further deepen our knowledge regarding the mechanisms through which TSP-1 influences microvascular reactivity following pulmonary MWCNT exposure. To better understand the regulatory role TSP-1 exerts on the microvasculature, we sought to investigate the role of the TSP-1 receptor CD47. Investigations of microvascular dysfunction post-nanomaterial inhalation strongly suggested that alterations in NO signaling and redox status were involved (Nurkiewicz et al., 2009, LeBlanc et al., 2010). With this information and knowing the involvement of CD47 in diseases associated with inflammation (Narizhneva et al., 2005, Salajegheh et al., 2005, Salajegheh et al., 2007) and NO disruption (Isenberg et al., 2008a, Isenberg et al., 2008c, Isenberg et al., 2009b, Bauer et al., 2010), we sought to investigate its role by utilizing CD47 KO mice for intravital and molecular experiments. Using the same intravital preparation as in Chapter 3, in Chapter 4 we measured arteriolar response to ACh in CD47 KO animals and observed a similar protection from MWCNT-induced impairment in vasodilation as in experiments with TSP-1 KO animals. Unfortunately, we were unable to obtain additional CD47 KO mice in which to conduct SNP experiments to test NO sensitivity. This is a limitation of this work, however there is little reason to expect them to behave differently from the TSP-1 KO mice, as the preserved TSP-1/CD36 signaling in the CD47KO animals has little direct impact on smooth muscle sGC activity (Isenberg et al., 2007).

TSP-1/CD47 signaling enhances leukocyte endothelial interaction through ICAM/VCAM activation (Narizhneva et al., 2005). We observed a more than two-fold increase in adhered or rolling leukocytes in venules in WT animals exposed to MWCNT. 
When apocynin was applied normal rolling activity was rescued. VCAM may also be upregulated by ROS stress (Deem and Cook-Mills, 2004). We also observed decreased tissue NOx in the WT + MWCNT group using the Sievers NO analyzer, while the opposite was true for CD47 and TSP1 KO animals. We were unable, however, to detect changes in blood ROS (using PPH spin trapping). Understood in the context of decreased NO mediated vasodilation observed in the WT + MWCNT, these findings are suggestive of decreased NO bioavailability, either due to depressed NO production, enhanced NO quenching by free radicals generated by NOX and eNOS uncoupling, or a combination of both being mediated by TSP-1/CD47 signaling.

Finally, in chapter 4 we also attempted to analyze tissue biomarkers of inflammation from the lung, into the circulation, and in the gluteus maximus tissue. The lack of significant differences between WT Sham and MWCNT groups is in contrast with some studies that have identified the same cytokines as elevated in the circulation and BAL fluid (Inoue et al., 2009, Han et al., 2010, Porter et al., 2010, Dong et al., 2015), but in agreement with others that have found no change (Mitchell et al., 2007, Elgrabli et al., 2008). It is possible that circulating inflammatory cytokines are generally higher in CD47 KO animals, possibly due to the anti-inflammatory role of TSP-1/CD47 interactions. CD47, TSP-1, and TSP-2 KO mice sustain some inflammatory responses to a greater degree than WT counterparts (Lamy et al., 2007) and CD47 signaling may inhibit cytokine production (Demeure et al., 2000). It may also be the case that CD47 KO animals have a similar cytokine response but differ temporaly from WT animals. Further investigation 
with a CD47 + Sham group and at different time points is warranted to provide more information about this question.

\section{Future Directions}

For as many questions as this project answers regarding the molecular mechanisms of microvascular dysfunction following MWCNT exposure, it raises even more to be answered in the future. The most obvious route of continued investigation would be further defining and characterizing the molecular pathways involved in the observed dysfunction. The observations of Aragon et al. 2017 suggest that CD36 is the necessary receptor for TSP-1 to exert influence on arterial reactivity. This raises a number of possibilities in terms of reconciling these findings with our work. However, our observation in chapter 3 that WT animals have reduced sensitivity to exogenously supplied NO suggests that CD47-mediated disruption of sGC activity is at least partly responsible for the observed dysfunction. It is possible that inhibition of either CD36 or CD47 is sufficient to offer vasoprotection. Owing to the different tissues observed between these studies (aorta vs. arterioles) it possible that CD36 signaling predominates in the compliance vessels, while CD47 is responsible for altering NO signaling in the arterioles. It may also be the case that in isolated vessels CD36 signaling is more effective, or the opposite is the case for CD47. To resolve the question of relative contribution between receptors, it would be valuable to evaluate endothelium dependent arteriolar responsiveness using intravital experiments on CD36 KO arterioles. Alternatively, in WT animals, oligonucleotides, peptides, siRNA, or other drugs could be delivered intravenously to block CD36 or CD47 signaling, possibly allowing for repeated measures 
comparisons between reactivity in the same animal (Isenberg et al., 2008b, Wang et al., 2013, Mansor et al., 2017). Once the contribution of receptors is determined it would next be advantageous to establish which downstream targets are most susceptible to disruption by CD47/CD36 signaling after MWCNT exposure.

The relationship between physico-chemical properties of the chosen particulate matter for exposure to TSP-1 is another potential area for investigations. Our findings of elevated peripheral TSP-1 following MWCNT exposure, but not following nano- $\mathrm{CeO}_{2}$, suggest that differences in size, aspect ratio, reactivity, chemical composition, and functionalization may differentially activate this signaling pathway. It would therefore be valuable to measure TSP-1 in tissues collected from a wide variety of nanomaterials. Observed protein levels could be compared to the properties of each material to search for potential correlations. Ultimately, this knowledge could be used to increase the safety of given nanomaterials by constraining their physical characteristics or by adding surface modifications to be less toxic.

The observations made in chapters 3 and 4 are limited to the 24-hour postexposure time point following a single MWCNT exposure. TSP-1 and arteriolar function should be measured in more occupationally-relevant chronic exposure regimen to truly assess the long term health effects. Chronic elevation of TSP-1 is associated with decreased skeletal muscle capillarity (Audet et al., 2013) as well as other cardiovascular pathologies (Roth et al., 1998, Maier et al., 2010, Choi et al., 2012). It is possible that chronic MWCNT exposure and TSP-1 elevation may exacerbate many of these diseases. 
These studies used the relatively large single dose of $50 \mathrm{ug}$, which is roughly equivalent to an entire career's worth of accumulated MWCNT exposure (Porter et al., 2010). In preliminary experiments, we also observed significant increases in peripheral TSP-1 after MWCNT aspiration of as little as $2.5 \mu \mathrm{g} /$ mouse (compared to $50 \mu \mathrm{g}$ used in chapters 3 and 4). To better understand the role of TSP-1 in this context, it is necessary to develop a dose-dependent relationship between MWCNT exposure level, tissue TSP-1 protein, and impairments of microvascular function. It is possible that there is a "safe" threshold of exposure to these materials that do not elicit a TSP-1 response. In addition to altering the dose of raw mitsui-7 MWCNT, it may also be worthwhile to investigate the role of MWCNT functionalization-the chemical addition of various hydrophilic groups that aid in solubility and dispersability-in generation of a TSP-1/CD47 response. Adding various functional groups, including $\mathrm{COOH}, \mathrm{NH}_{2}$, and polyethylene glycol to the surface of MWCNT alters their physicochemical properties accordant pulmonary toxicity. Compared to raw MWCNT, functionalized MWNCT exposure may result in more, less, or roughly equal measures of lung toxicity (Li et al., 2013). Microvascular complications of exposure to these materials has seen little investigation, and it is possible that they may differentially elicit TSP-1/CD47 signaling compared to raw MWCNT.

One pressing question that we were unable to answer is identifying the source of TSP-1 following MWCNT exposure. TSP-1, as discussed in Chapter 2, is a major component of platelets and makes up as much as $25 \%$ of all proteins secreted by activated platelets (Switalska et al., 1985) and enhanced levels in some cases of lung injury and inflammation (Idell et al., 1989). If activated platelets are one possible source 
for secreted TSP-1 inhibiting platelet activation pharmacologically before and following MWCNT exposure should prevent a rise in TSP-1 levels. Choice of inhibitor in this case would be critical, as many have vasoactive side effects. If activated platelets are not a major source of TPS-1, the Campen group has postulated that elevated MMP9 activity following lung exposure results in extracellular matrix remodeling (Aragon et al., 2016). This remodeling process releases TSP-1 and possibly small CD36/CD47 binding peptides into the circulation, resulting in systemic effects. (Aragon et al., 2016). In these experiments the researchers observed increased MMP-9 in MWNCT-exposed lung tissue, and a degree of protection from impairments in ACh mediated vasodilation in vessels incubated in serum from MMP-9 KO mice, however they did not measure lung or circulating TSP-1 levels. If this hypothesis is correct, TSP-1 levels in MMP-9 animals exposed to MWCNT should be lower than comparable WT animals.

Assuming that TSP-1 signaling truly is a mediator of microvascular dysfunction after MWCNT, inhibition of this pathway should offer WT animals a degree of protection comparable to TSP-1 and CD47 KO animals. Pharmacologic inhibition of TSP-1/CD47 signaling has been shown to increase tissue blood flow, NO production, angiogenesis, and ischemic survival (Isenberg et al., 2009b). It is important to note, however that inhibition of TSP-1 signaling may have the unintended consequence of promoting angiogenesis and tumor growth (Lawler et al., 2001). It is possible that this effect may be largely CD36-dependent (Lawler, 2002). If it can be determined that only CD47 signaling is necessary for MWCNT-induced impairments in arteriolar function, it may be preferable to target that receptor rather than inhibiting all TSP-1 signaling. 
One important aspect of cardiovascular physiology that was not investigated in this dissertation is the contribution of autonomic nerve activity to altered vascular reactivity which may be altered by pulmonary nanomaterial exposure (Stapleton et al., 2015a). The arterioles are innervated by postganglionic sympathetic neurons, which signal for vasoconstriction via the release of the catecholamine norepinephrine, which signals for vasoconstriction through smooth muscle a-adrenoceptors (Korthuis, 2011). Several measures of sympathetic outflow following pulmonary exposure to a variety of particulate matter have been investigated. Decreased heart rate variability, a measure of sympathetic outflow and decreased vagal tone, has been observed in workers exposed to $\mathrm{PM}_{2.5}$ and may persist for as long as 14 hours post-exposure (Cavallari et al., 2008a, Cavallari et al., 2008b). Pulmonary exposure to $10 \mu \mathrm{g}$ of nano- $\mathrm{TiO}_{2}$ resulted in persistent sympathetically mediated arteriolar dysfunction as measured by active hyperemia in the presence of a-adrenergic blockade (Knuckles et al., 2013). TSP-1 can augment acute vascular responses to epinephrine (Isenberg et al., 2009a) and phenylephrine (Mandler et al., 2014). It is possible altered sympathetic outflow may be contributing to some of the TSP-1/CD47-driven arteriolar effects observed in chapters 3 and 4 . It would be valuable to determine the contribution of sympathetic activity using pharmacologic agent such as phentolamine to block a-adrenergic receptor input in TSP-1 animals exposed to MWCNT. These experiments could be performed under baseline conditions as well as during iontophoretic stimulation using phenylephrine.

The findings presented this dissertation serve to further extend our knowledge of the molecular mechanisms underpinning the vascular consequences of pulmonary 
exposure to particulate matter, an area of research that will be critical in fully understanding the toxicology of these materials. As discussed previously, our findings are limited to a single ENM species, route of exposure, and time point, but should serve as a foundation for future investigations examining these variables. Combined with the growing body of literature linking TSP-1/CD47 signaling to a variety of cardiovascular pathologies, our findings suggest that this signaling axis should be investigated thoroughly in future work regarding ENM exposure.

\section{References}

Aragon, M., Erdely, A., Bishop, L., Salmen, R., Weaver, J., Liu, J., Hall, P., Eye, T., Kodali, V., Zeidler-Erdely, P., Stafflinger, J.E., Ottens, A.K. \& Campen, M.J., 2016. Mmp-9-Dependent Serum-Borne Bioactivity Caused By Multiwalled Carbon Nanotube Exposure Induces Vascular Dysfunction Via The Cd36 Scavenger Receptor. Toxicological Sciences, 150, 488-498.

Aragon, M.J., Topper, L., Tyler, C.R., Sanchez, B., Zychowski, K., Young, T., Herbert, G., Hall, P., Erdely, A., Eye, T., Bishop, L., Saunders, S.A., Muldoon, P.P., Ottens, A.K. \& Campen, M.J., 2017. Serum-Borne Bioactivity Caused By Pulmonary Multiwalled Carbon Nanotubes Induces Neuroinflammation Via Blood-Brain Barrier Impairment. Proceedings Of The National Academy Of Sciences, 114, E1968.

Audet, G.N., Fulks, D., Stricker, J.C. \& Olfert, I.M., 2013. Chronic Delivery Of A Thrombospondin-1 Mimetic Decreases Skeletal Muscle Capillarity In Mice. Plos One, 8.

Bagher, P., Polo-Parada, L. \& Segal, S.S., 2011. Microiontophoresis And Micromanipulation For Intravital Fluorescence Imaging Of The Microcirculation. Journal Of Visualized Experiments : Jove.

Bauer, E.M., Qin, Y., Miller, T.W., Bandle, R.W., Csanyi, G., Pagano, P.J., Bauer, P.M., Schnermann, J., Roberts, D.D. \& Isenberg, J.S., 2010. Thrombospondin-1 Supports Blood Pressure By Limiting Enos Activation And Endothelial-Dependent Vasorelaxation. Cardiovascular Research, 88, 471-481.

Bearden, S.E., Payne, G.W., Chisty, A. \& Segal, S.S., 2004. Arteriolar Network Architecture And Vasomotor Function With Ageing In Mouse Gluteus Maximus Muscle. Journal Of Physiology-London, 561, 535-545. 
Bolognese, L., Carrabba, N., Parodi, G., Santoro, G.M., Buonamici, P., Cerisano, G. \& Antoniucci, D., 2004. Impact Of Microvascular Dysfunction On Left Ventricular Remodeling And Long-Term Clinical Outcome After Primary Coronary Angioplasty For Acute Myocardial Infarction. Circulation, 109, 1121-1126.

Brevetti, G., Schiano, V. \& Chiariello, M., 2008. Endothelial Dysfunction: A Key To The Pathophysiology And Natural History Of Peripheral Arterial Disease? Atherosclerosis, 197, 1-11.

Cavallari, J.M., Eisen, E.A., Fang, S.C., Schwartz, J., Hauser, R., Herrick, R.F. \& Christiani, D.C., 2008a. Pm(2.5) Metal Exposures And Nocturnal Heart Rate Variability: A Panel Study Of Boilermaker Construction Workers. Environmental Health, 7.

Cavallari, J.M., Fang, S.C., Eisen, E.A., Schwartz, J., Hauser, R., Herrick, R.F. \& Christiani, D.C., 2008b. Time Course Of Heart Rate Variability Decline Following Particulate Matter Exposures In An Occupational Cohort. Inhalation Toxicology, 20, 415-422.

Choi, K.Y., Kim, D.B., Kim, M.J., Kwon, B.J., Chang, S.Y., Jang, S.W., Cho, E.J., Rho, T.H. \& Kim, J.H., 2012. Higher Plasma Thrombospondin-1 Levels In Patients With Coronary Artery Disease And Diabetes Mellitus. Korean Circulation Journal, 42, 100-106.

Deem, T.L. \& Cook-Mills, J.M., 2004. Vascular Cell Adhesion Molecule 1 (Vcam-1) Activation Of Endothelial Cell Matrix Metalloproteinases: Role Of Reactive Oxygen Species. Blood, 104, 2385-2393.

Demeure, C.E., Tanaka, H., Mateo, V., Rubio, M., Delespesse, G. \& Sarfati, M., 2000. Cd47 Engagement Inhibits Cytokine Production And Maturation Of Human Dendritic Cells. Journal Of Immunology, 164, 2193-2199.

Dong, J., Porter, D.W., Batteli, L.A., Wolfarth, M.G., Richardson, D.L. \& Ma, Q., 2015. Pathologic And Molecular Profiling Of Rapid-Onset Fibrosis And Inflammation Induced By Multi-Walled Carbon Nanotubes. Archives Of Toxicology, 89, 621633.

Elgrabli, D., Abella-Gallart, S., Robidel, F., Rogerieux, F., Boczkowski, J. \& Lacroix, G., 2008. Induction Of Apoptosis And Absence Of Inflammation In Rat Lung After Intratracheal Instillation Of Multiwalled Carbon Nanotubes. Toxicology, 253, 131136.

Frisbee, J.C. \& Stepp, D.W., 2001. Impaired No-Dependent Dilation Of Skeletal Muscle Arterioles In Hypertensive Diabetic Obese Zucker Rats. American Journal Of Physiology-Heart And Circulatory Physiology, 281, H1304-H1311.

Givvimani, S., Narayanan, N., Armaghan, F., Pushpakumar, S. \& Tyagi, S.C., 2013. Attenuation Of Conducted Vasodilation In Skeletal Muscle Arterioles During Hyperhomocysteinemia. Pharmacology, 91, 287-296.

Han, S.G., Andrews, R. \& Gairola, C.G., 2010. Acute Pulmonary Response Of Mice To Multi-Wall Carbon Nanotubes. Inhalation Toxicology, 22, 340-347.

Hatoum, O.A., Binion, D.G., Otterson, M.F. \& Gutterman, D.D., 2003. Acquired Microvascular Dysfunction In Inflammatory Bowel Disease: Loss Of Nitric OxideMediated Vasodilation. Gastroenterology, 125, 58-69. 
Iadecola, C., Zhang, F.Y., Niwa, K., Eckman, C., Turner, S.K., Fischer, E., Younkin, S., Borchelt, D.R., Hsiao, K.K. \& Carlson, G.A., 1999. Sod1 Rescues Cerebral Endothelial Dysfunction In Mice Overexpressing Amyloid Precursor Protein. Nature Neuroscience, 2, 157-161.

Idell, S., Maunder, R., Fein, A.M., Switalska, H.I., Tuszynski, G.P., Mclarty, J. \& Niewiarowski, S., 1989. Platelet-Specific Alpha-Granule Proteins And Thrombospondin In Bronchoalveolar Lavage In The Adult Respiratory-Distress Syndrome. Chest, 96, 1125-1132.

Inoue, K., Koike, E., Yanagisaw, R., Hirano, S., Nishikawa, M. \& Takano, H., 2009. Effects Of Multi-Walled Carbon Nanotubes On A Murine Allergic Airway Inflammation Model. Toxicology And Applied Pharmacology, 237, 306-316.

Isenberg, J.S., Frazier, W.A. \& Roberts, D.D., 2008a. Thrombospondin-1: A Physiological Regulator Of Nitric Oxide Signaling. Cellular And Molecular Life Sciences, 65, 728-742.

Isenberg, J.S., Jia, Y.F., Fukuyama, J., Switzer, C.H., Wink, D.A. \& Roberts, D.D., 2007. Thrombospondin-1 Inhibits Nitric Oxide Signaling Via Cd36 By Inhibiting Myristic Acid Uptake. Journal Of Biological Chemistry, 282, 15404-15415.

Isenberg, J.S., Pappan, L.K., Romeo, M.J., Abu-Asab, M., Tsokos, M., Wink, D.A., Frazier, W.A. \& Roberts, D.D., 2008b. Blockade Of Thrombospondin-1-Cd47 Interactions Prevents Necrosis Of Full Thickness Skin Grafts. Annals Of Surgery, 247, 180-190.

Isenberg, J.S., Qin, Y., Maxhimer, J.B., Sipes, J.M., Despres, D., Schnermann, J., Frazier, W.A. \& Roberts, D.D., 2009a. Thrombospondin-1 And Cd47 Regulate Blood Pressure And Cardiac Responses To Vasoactive Stress. Matrix Biology, 28, 110-119.

Isenberg, J.S., Romeo, M.J., Yu, C., Yu, C.K., Nghiem, K., Monsale, J., Rick, M.E., Wink, D.A., Frazier, W.A. \& Roberts, D.D., 2008c. Thrombospondin-1 Stimulates Platelet Aggregation By Blocking The Antithrombotic Activity Of Nitric Oxide/Cgmp Signaling. Blood, 111, 613-623.

Isenberg, J.S., Shiva, S. \& Gladwin, M., 2009b. Thrombospondin-1-Cd47 Blockade And Exogenous Nitrite Enhance Ischemic Tissue Survival, Blood Flow And Angiogenesis Via Coupled No-Cgmp Pathway Activation. Nitric Oxide-Biology And Chemistry, 21, 52-62.

Kampfrath, T., Maiseyeu, A., Ying, Z.K., Shah, Z., Deiuliis, J.A., Xu, X.H., Kherada, N., Brook, R.D., Reddy, K.M., Padture, N.P., Parthasarathy, S., Chen, L.C., MoffattBruce, S., Sun, Q.H., Morawietz, H. \& Rajagopalan, S., 2011. Chronic Fine Particulate Matter Exposure Induces Systemic Vascular Dysfunction Via Nadph Oxidase And TIr4 Pathways. Circulation Research, 108, 716-U400.

Knuckles, T.L., Stapleton, P.A., Minarchick, V.C., Esch, L., Mccawley, M., Hendryx, M. \& Nurkiewicz, T.R., 2013. Air Pollution Particulate Matter Collected From An Appalachian Mountaintop Mining Site Induces Microvascular Dysfunction. Microcirculation, 20, 158-169. 
Koller, A. \& Huang, A., 1994. Impaired Nitric Oxide-Mediated Flow-Induced Dilation In Arterioles Of Spontaneously Hypertensive Rats. Circulation Research, 74, 416421.

Korthuis, R., 2011. Regulation Of Vascular Tone In Skeletal Muscle. Skeletal Muscle Circulation. San Rafael, Ca: Morgan \& Claypool Life Sciences.

Labrousse-Arias, D., Castillo-Gonzalez, R., Rogers, N.M., Torres-Capelli, M., Barreira, B., Aragones, J., Cogolludo, A., Isenberg, J.S. \& Calzada, M.J., 2016. Hif-2 AlphaMediated Induction Of Pulmonary Thrombospondin-1 Contributes To HypoxiaDriven Vascular Remodelling And Vasoconstriction. Cardiovascular Research, $109,115-130$.

Lamy, L., Foussat, A., Brown, E., Bornstein, P., Ticchioni, M. \& Bernard, A., 2007. Interactions Between $\mathrm{Cd} 47$ And Thrombospondin Reduce Inflammation. Journal Of Immunology, 178, 5930-5939.

Lawler, J., 2002. Thrombospondin-1 As An Endogenous Inhibitor Of Angiogenesis And Tumor Growth. Journal Of Cellular And Molecular Medicine, 6, 1-12.

Lawler, J., Miao, W.M., Duquette, M., Bouck, N., Bronson, R.T. \& Hynes, R.O., 2001. Thrombospondin-1 Gene Expression Affects Survival And Tumor Spectrum Of P53-Deficient Mice. American Journal Of Pathology, 159, 1949-1956.

Leblanc, A.J., Cumpston, J.L., Chen, B.T., Frazer, D., Castranova, V. \& Nurkiewicz, T.R., 2009. Nanoparticle Inhalation Impairs Endothelium-Dependent Vasodilation In Subepicardial Arterioles. Journal Of Toxicology And Environmental Health-Part ACurrent Issues, 72, 1576-1584.

Leblanc, A.J., Moseley, A.M., Chen, B.T., Frazer, D., Castranova, V. \& Nurkiewicz, T.R., 2010. Nanoparticle Inhalation Impairs Coronary Microvascular Reactivity Via A Local Reactive Oxygen Species-Dependent Mechanism. Cardiovascular Toxicology, 10, 27-36.

Li, R.B., Wang, X., Ji, Z.X., Sun, B.B., Zhang, H.Y., Chang, C.H., Lin, S.J., Meng, H., Liao, Y.P., Wang, M.Y., Li, Z.X., Hwang, A.A., Song, T.B., Xu, R., Yang, Y., Zink, J.I., Nel, A.E. \& Xia, T., 2013. Surface Charge And Cellular Processing Of Covalently Functionalized Multiwall Carbon Nanotubes Determine Pulmonary Toxicity. Acs Nano, 7, 2352-2368.

Maier, K.G., Han, X., Sadowitz, B., Gentile, K.L., Middleton, F.A. \& Gahtan, V., 2010. Thrombospondin-1: A Proatherosclerotic Protein Augmented By Hyperglycemia. Journal Of Vascular Surgery, 51, 1238-1247.

Mandler, W.K., Nurkiewicz, T. \& Olfert, I.M., 2014. Thrombospondin-1 Null Mice Exhibit Enhanced Microvessel Endothelium Dependent Vasodilation, Reduced Adrenergic Sensitivity. Faseb Journal, 28.

Mandler, W.K., Nurkiewicz, T.R., Porter, D.W. \& Olfert, I.M., 2017. Thrombospondin-1 Mediates Multi-Walled Carbon Nanotube Induced Impairment Of Arteriolar Dilation. Nanotoxicology, 11, 112-122.

Mansor, L.S., Sousa Fialho, M.D.L., Yea, G., Coumans, W.A., West, J.A., Kerr, M., Carr, C.A., Luiken, J.J.F.P., Glatz, J.F.C., Evans, R.D., Griffin, J.L., Tyler, D.J., Clarke, K. \& Heather, L.C., 2017. Inhibition Of Sarcolemmal Fat/Cd36 By Sulfo-N- 
Succinimidyl Oleate Rapidly Corrects Metabolism And Restores Function In The Diabetic Heart Following Hypoxia/Reoxygenation. Cardiovasc Res.

Matsuo, Y., Tanaka, M., Yamakage, H., Sasaki, Y., Muranaka, K., Hata, H., Ikai, I., Shimatsu, A., Inoue, M., Chun, T.H. \& Satoh-Asahara, N., 2015. Thrombospondin 1 As A Novel Biological Marker Of Obesity And Metabolic Syndrome. MetabolismClinical And Experimental, 64, 1490-1499.

Meller, S.M., Stilp, E., Walker, C.N. \& Mena-Hurtado, C., 2013. The Link Between Vasculogenic Erectile Dysfunction, Coronary Artery Disease, And Peripheral Artery Disease: Role Of Metabolic Factors And Endovascular Therapy. Journal Of Invasive Cardiology, 25, 313-319.

Michaels, A.D., Gibson, C.M. \& Barron, H.V., 2000. Microvascular Dysfunction In Acute Myocardial Infarction: Focus On The Roles Of Platelet And Inflammatory Mediators In The No-Reflow Phenomenon. American Journal Of Cardiology, 85, 50b-60b.

Mitchell, L.A., Gao, J., Wal, R.V., Gigliotti, A., Burchiel, S.W. \& Mcdonald, J.D., 2007. Pulmonary And Systemic Immune Response To Inhaled Multiwalled Carbon Nanotubes. Toxicological Sciences, 100, 203-214.

Morrell, N.W., Adnot, S., Archer, S.L., Dupuis, J., Jones, P.L., Maclean, M.R., Mcmurtry, I.F., Stenmark, K.R., Thistlethwaite, P.A., Weissmann, N., Yuan, J.X.J. \& Weir, E.K., 2009. Cellular And Molecular Basis Of Pulmonary Arterial Hypertension. Journal Of The American College Of Cardiology, 54, S20-S31.

Narizhneva, N.V., Razorenova, O.V., Podrez, E.A., Chen, J.H., Chandrasekharan, U.M., Dicorleto, P.E., Plow, E.F., Topol, E.J. \& Byzova, T.V., 2005. Thrombospondin-1 Up-Regulates Expression Of Cell Adhesion Molecules And Promotes Monocyte Binding To Endothelium. Faseb Journal, 19, 1158-+.

Nurkiewicz, T.R., Porter, D.W., Barger, M., Castranova, V. \& Boegehold, M.A., 2004. Particulate Matter Exposure Impairs Systemic Microvascular EndotheliumDependent Dilation. Environmental Health Perspectives, 112, 1299-1306. Nurkiewicz, T.R., Porter, D.W., Barger, M., Millecchia, L., Rao, K.M.K., Marvar, P.J., Hubbs, A.F., Castranova, V. \& Boegehold, M.A., 2006. Systemic Microvascular Dysfunction And Inflammation After Pulmonary Particulate Matter Exposure. Environmental Health Perspectives, 114, 412-419.

Nurkiewicz, T.R., Porter, D.W., Hubbs, A.F., Cumpston, J.L., Chen, B.T., Frazer, D.G. \& Castranova, V., 2008. Nanoparticle Inhalation Augments Particle-Dependent Systemic Microvascular Dysfunction. Particle And Fibre Toxicology, 5.

Nurkiewicz, T.R., Porter, D.W., Hubbs, A.F., Stone, S., Chen, B.T., Frazer, D.G., Boegehold, M.A. \& Castranova, V., 2009. Pulmonary Nanoparticle Exposure Disrupts Systemic Microvascular Nitric Oxide Signaling. Toxicological Sciences, 110, 191-203.

Porter, D.W., Hubbs, A.F., Mercer, R.R., Wu, N.Q., Wolfarth, M.G., Sriram, K., Leonard, S., Battelli, L., Schwegler-Berry, D., Friend, S., Andrew, M., Chen, B.T., Tsuruoka, S., Endo, M. \& Castranova, V., 2010. Mouse Pulmonary Dose- And Time Course-Responses Induced By Exposure To Multi-Walled Carbon Nanotubes. Toxicology, 269, 136-147. 
Roth, J.J., Gahtan, V., Brown, J.L., Gerhard, C., Swami, V.K., Rothman, V.L., Tulenko, T.N. \& Tuszynski, G.P., 1998. Thrombospondin-1 Is Elevated With Both Intimal Hyperplasia And Hypercholesterolemia. Journal Of Surgical Research, 74, 11-16. Salajegheh, M., Raju, R., Schmidt, J. \& Dalakas, M.C., 2005. Thrombospondin-1 (Tsp1) And Its Binding Partners Cd36 And Cd47, As Mediators Of Inflammation In Sporadic Inclusion Body Myositis (Sibm). Neurology, 64, A158-A158.

Salajegheh, M., Raju, R., Schmidt, J. \& Dalakas, M.C., 2007. Upregulation Of Thrombospondin-1(Tsp-1) And Its Binding Partners, Cd36 And Cd47, In Sporadic Inclusion Body Myositis. Journal Of Neuroimmunology, 187, 166-174.

Sinkler, S.Y. \& Segal, S.S., 2014. Aging Alters Reactivity Of Microvascular Resistance Networks In Mouse Gluteus Maximus Muscle. American Journal Of PhysiologyHeart And Circulatory Physiology, 307, H830-H839.

Smadja, D.M., D'audigier, C., Bieche, I., Evrard, S., Mauge, L., Dias, J.V., Labreuche, J., Laurendeau, I., Marsac, B., Dizier, B., Wagner-Ballon, O., Boisson-Vidal, C., Morandi, V., Duong-Van-Huyen, J.P., Bruneval, P., Dignat-George, F., Emmerich, J. \& Gaussem, P., 2011. Thrombospondin-1 Is A Plasmatic Marker Of Peripheral Arterial Disease That Modulates Endothelial Progenitor Cell Angiogenic Properties. Arteriosclerosis Thrombosis And Vascular Biology, 31, 551-559.

Stapleton, P.A., Abukabda, A.B., Hardy, S.L. \& Nurkiewicz, T.R., 2015a. Xenobiotic Pulmonary Exposure And Systemic Cardiovascular Response Via Neurological Links. American Journal Of Physiology-Heart And Circulatory Physiology, 309, $\mathrm{H} 1609-\mathrm{H} 1620$.

Stapleton, P.A., Mcbride, C.R., Yi, J. \& Nurkiewicz, T.R., 2015b. Uterine Microvascular Sensitivity To Nanomaterial Inhalation: An In Vivo Assessment. Toxicology And Applied Pharmacology, 288, 420-428.

Stapleton, P.A., Minarchick, V.C., Cumpston, A.M., Mckinney, W., Chen, B.T., Sager, T.M., Frazer, D.G., Mercer, R.R., Scabilloni, J., Andrew, M.E., Castranova, V. \& Nurkiewicz, T.R., 2012. Impairment Of Coronary Arteriolar EndotheliumDependent Dilation After Multi-Walled Carbon Nanotube Inhalation: A TimeCourse Study. International Journal Of Molecular Sciences, 13, 13781-13803.

Stenina, O.I., Krukovets, I., Wang, K., Zhou, Z.M., Forudi, F., Penn, M.S., Topol, E.J. \& Plow, E.F., 2003. Increased Expression Of Thrombospondin-1 In Vessel Wall Of Diabetic Zucker Rat. Circulation, 107, 3209-3215.

Sun, Q.H., Yue, P., Deiuliis, J.A., Lumeng, C.N., Kampfrath, T., Mikolaj, M.B., Cai, Y., Ostrowski, M.C., Lu, B., Parthasarathy, S., Brook, R.D., Moffatt-Bruce, S.D., Chen, L.C. \& Rajagopalan, S., 2009. Ambient Air Pollution Exaggerates Adipose Inflammation And Insulin Resistance In A Mouse Model Of Diet-Induced Obesity. Circulation, 119, 538-U91.

Switalska, H.I., Niewiarowski, S., Tuszynski, G.P., Rucinski, B., Schmaier, A.H., Morinelli, T.A. \& Cierniewski, C.S., 1985. Radioimmunoassay Of Human-Platelet Thrombospondin - Different Patterns Of Thrombospondin And BetaThromboglobulin Antigen Secretion And Clearance From The Circulation. Journal Of Laboratory And Clinical Medicine, 106, 690-700. 
Varma, V., Yao-Borengasser, A., Bodles, A.M., Rasouli, N., Phanavanh, B., Nolen, G.T., Kern, E.M., Nagarajan, R., Spencer, H.J., Lee, M.J., Fried, S.K., Mcgehee, R.E., Peterson, C.A. \& Kern, P.A., 2008. Thrombospondin-1 Is An Adipokine Associated With Obesity, Adipose Inflammation, And Insulin Resistance. Diabetes, 57, 432439.

Wang, Y., Xu, Z., Guo, S., Zhang, L., Sharma, A., Robertson, G.P. \& Huang, L., 2013. Intravenous Delivery Of Sirna Targeting Cd47 Effectively Inhibits Melanoma Tumor Growth And Lung Metastasis. Mol Ther, 21, 1919-29.

Xu, X.H., Rao, X.Q., Wang, T.Y., Jiang, S.Y., Ying, Z.K., Liu, C.Q., Wang, A.X., Zhong, M.H., Deiuliis, J.A., Maiseyeu, A., Rajagopalan, S., Lippmann, M., Chen, L.C. \& Sun, Q.H., 2012. Effect Of Co-Exposure To Nickel And Particulate Matter On Insulin Resistance And Mitochondrial Dysfunction In A Mouse Model. Particle And Fibre Toxicology, 9.

Xu, X.H., Yavar, Z.B., Verdin, M., Ying, Z.K., Mihai, G., Kampfrath, T., Wang, A.X., Zhong, M.H., Lippmann, M., Chen, L.C., Rajagopalan, S. \& Sun, Q.H., 2010. Effect Of Early Particulate Air Pollution Exposure On Obesity In Mice Role Of P47(Phox). Arteriosclerosis Thrombosis And Vascular Biology, 30, 2518-U357. 